\title{
Phase Equilibria as Related to Crystal Structure in the System Niobium Pentoxide-Tungsten Trioxide
}

\author{
R. S. Roth and J. L. Waring
}

Institute for Materials Research, Narional Bureau of Standards, Washington, D.C.

(April 1, 1966)

\begin{abstract}
The phase equilibrium diagram for the binary system niobium pentoxide-tungsten trioxide has been constructed from results of x-ray diffraction studies on both single crystals and powders and from fusion characteristics. Twelve stable compounds have been found in the system. The exact composition of eight of these compounds has been established by single crystal analyses at the $\mathrm{Nb}_{2} \mathrm{O}_{5}: \mathrm{WO}_{3}$ ratios of $6: 1,13: 4,7: 3,8: 5,9: 8,1: 1,4: 9$, and $2: 7$. The approximate compositions of the remaining four other phases are $30: 1,6: 11,1: 11$, and $1: 15$. The $6: 1$, " $6: 11$ ", $4: 9$, and $2: 7$ phases melt congruently at $1476,1378,1380$, and $1357^{\circ} \mathrm{C}$, respectively. The " $30: 1$ ", $7: 3,8: 5,9: 8$, " $1: 11 "$, and " $1: 15 "$ phases melt incongruently at $1470,1440,1385,1375,1356$, and $1358{ }^{\circ} \mathrm{C}$, respectively; and the $13: 4$ and $1: 1$ phases decompose before melting at 1435 and $1115^{\circ} \mathrm{C}$, respectively. The $8: 5,9: 8, " 6: 11 ", 2: 7, " 1: 11 "$, and "1:15" compounds are shown on the phase diagram as having minimum temperatures of stability. One metastable phase having a narrow range of composition near the $3: 8$ ratio was also encountered. Although $\mathrm{Nb}_{2} \mathrm{O}_{5}$ apparently exhibits no solid solution, $\mathrm{WO}_{3}$ was found to accept a maximum of three mole percent niobia in solid solution enabling all the reported polymorphs of $\mathrm{WO}_{3}$ to be obtained at room temperature.
\end{abstract}

Key Words: Crystal structure, niobium pentoxide, phase equilibria, system, tungsten trioxide.

\section{Introduction}

A complete study of the phase relationships in the binary system $\mathrm{Nb}_{2} \mathrm{O}_{5}-\mathrm{WO}_{3}$ has been conducted as part of a continuing program of fundamental phase equilibria studies of ceramic materials. This particular system was selected for study in order to further the knowledge of the crystal chemistry of niobates in view of the results obtained from previous structural studies of single crystals [1-4]. ${ }^{1}$

Due to the complex nature of the x-ray diffraction powder patterns in this system, conflicting interpretations of the data have been reported [5-11]. In a previous study of the $\mathrm{Nb}_{2} \mathrm{O}_{5}-\mathrm{WO}_{3}$ system, Goldschmidt [5] concluded that $\mathrm{Nb}_{2} \mathrm{O}_{5}$ could accept more than 50 mole percent $\mathrm{WO}_{3}$ in solid solution and reported the existence of a compound at approximately $\mathrm{Nb}_{2} \mathrm{O}_{5}: 3 \mathrm{WO}_{3}$. He also found a limited solid solution of $\mathrm{Nb}_{2} \mathrm{O}_{5}$ in $\mathrm{WO}_{3}$. Kovba and Trunov [6] reported that the $1: 3$ compound was tetragonal with a structure related to that of a tetragonal tungsten-bronze. Fiegel et al. [7], studied the phase equilibria in the system at $1200{ }^{\circ} \mathrm{C}$ and below. They reported only about 33 mole percent solid solution of $\mathrm{WO}_{3}$ in $\mathrm{Nb}_{2} \mathrm{O}_{5}$ with a $3: 2$ compound occurring at $1200{ }^{\circ} \mathrm{C}$ and a $1: 1$ compound at $1100{ }^{\circ} \mathrm{C}$ and below. They found very little, if any, solid solution of $\mathrm{Nb}_{2} \mathrm{O}_{5}$ in $\mathrm{WO}_{3}$ and confirmed the existence of a compound at about $1: 3$ with a limited solid solution for both $\mathrm{Nb}_{2} \mathrm{O}_{5}$ and $\mathrm{WO}_{3}$.

' Figures in brackets indicate the literature references at the end of this paper.
Later [8] they reported that the $1: 3$ composition gave single crystal $x$-ray diffraction patterns which showed superstructure differing from one crystal to the next. Kovba et al. [9], also attempted to determine the phases formed in this system at about $1200^{\circ} \mathrm{C}$. They concluded that compounds existed at the $\mathrm{Nb}_{2} \mathrm{O}_{5}: \mathrm{WO}_{3}$ ratios of $4: 1,2: 1,4: 7$, and $1: 3$. They also, reported a phase with variable $\mathrm{WO}_{3}$ content occurring at high $\mathrm{WO}_{3}$ concentrations and observed a small amount of solid solution in both $\mathrm{Nb}_{2} \mathrm{O}_{5}$ and $\mathrm{WO}_{3}$.

In a description of preliminary phase identification, Roth and Wadsley [1] on the basis of single crystal $\mathrm{x}$-ray diffraction studies reported the existence of five compounds structurally related to $\mathrm{Nb}_{2} \mathrm{O}_{5}$ occurring at $\mathrm{Nb}_{2} \mathrm{O}_{5}: \mathrm{WO}_{3}$ ratios of $15: 1,6: 1,7: 3,8: 5$, and $9: 8$. They also confirmed the $1: 1$ phase and noted the existence of at least three phases related to the tetragonal tungsten bronze-type structure. The crystal structures of $6: 1,7: 3,8: 5$, and $9: 8$, were reported [2,3] and the "building block" principle was elaborated as the basis of the crystal-chemistry of niobate compounds [4].

Schäfer and Gruehn [10] and Felten [11] have indicated in private communications that their interpretations of x-ray diffraction powder data for the $\mathrm{Nb}_{2} \mathrm{O}_{5}-\mathrm{WO}_{3}$ system differ in some respects from the previously mentioned published reports.

Because of the conflicting nature of the reported data, it was thought desirable to study completely the phase equilibria of the $\mathrm{Nb}_{2} \mathrm{O}_{5}-\mathrm{WO}_{3}$ system. With the use of the unit cell dimensions derived from 
single crystal data the existence of the various phases in the powder patterns can now be established more readily. The exact compositions of phases can often be determined only by a solution of the crystal structure. The approximate composition and even the existence of a phase can sometimes be found only by a rather complete phase equilibria study. The two disciplines, crystal structure analysis and phase equilibria are therefore complimentary and no system can be considered to be well characterized unless both such studies have been made. Therefore, x-ray diffraction data together with the melting points of the compounds and solidus and liquidus temperatures at various compositions across the system have been obtained in order to construct an equilibrium diagram.

\section{Sample Preparation and Test Methods}

The following starting materials were employed for the preparation of the majority of the specimens:

$\mathrm{Nb}_{2} \mathrm{O}_{5}$ - high purity niobium pentoxide. Spectrographic analysis indicated less than about 0.01 percent $\mathrm{Si} ; 0.001$ percent $\mathrm{Ca}$ and $\mathrm{Mg}$ with $\mathrm{As}, \mathrm{Cu}$, and Ta only questionably present.

$\mathrm{WO}_{3}$ - high purity tungsten anhydride. Spectrographic analysis indicated less than about 0.1 percent $\mathrm{Si}$; 0.001 percent $\mathrm{B}, \mathrm{Ca}$, $\mathrm{Cr}$, and $\mathrm{Mg} ; 0.0001$ percent $\mathrm{Cu}$ with $\mathrm{Pb}$ only questionably present.

A few specimens were prepared with less pure starting materials, in order to determine qualitatively the effect of impurities on the equilibrium products. The following is a typical example of the nature of the impurities present in two specimens of $\mathrm{Nb}_{2} \mathrm{O}_{5}: \mathrm{WO}_{3}$ ratio of $16: 5$. For the less pure specimen the first series transition elements, in general, are present in amounts of an order of magnitude greater than for the more pure specimen.

$\mathrm{Nb}_{2} \mathrm{O}_{5}: \mathrm{WO}_{3}(16: 5)$-higher purity end members. Spectrochemical analyses indicated less than about 0.01 percent $\mathrm{Cr}, \mathrm{Cu}$, and $\mathrm{Si} ; 0.001$ percent $\mathrm{Al}, \mathrm{B}, \mathrm{Ca}, \mathrm{Mg}$, and $\mathrm{Ni}$; 0.0001 percent $\mathrm{Mn}$ with $\mathrm{Pb}$ only questionably present. $\mathrm{Nb}_{2} \mathrm{O}_{5}: \mathrm{WO}_{3}(16: 5)$ - less pure end members. Spectrochemical analyses indicated less than about 0.01 percent $\mathrm{Al}, \mathrm{Ca}, \mathrm{Cr}, \mathrm{Cu}, \mathrm{Fe}$, $\mathrm{Mg}, \mathrm{Ni}$, and $\mathrm{Si} ; 0.001$ percent Mn; 0.0001 percent $B$ with $\mathrm{Pb}$ only questionably present.

For the preparation of the specimens, the weight percentages were calculated to within \pm 0.01 percent, with no corrections made for percentage of purity of the starting materials except for loss on ignition.

For the higher purity specimens, the starting materials were weighed to the nearest $\pm 0.1 \mathrm{mg}$, in sufficient quantities to yield $3 \mathrm{~g}$ batches. Each batch was mixed in a mechanical shaker for about
15 min and pressed into a disk in a 5/8-in. diam mold at $10,000 \mathrm{lb} / \mathrm{in}^{2}$. The disks were placed on $\mathrm{Pt}$ foil and calcined by heating in air at $700{ }^{\circ} \mathrm{C}$ for $19 \mathrm{hrs}$, with heating and cooling rates of approximately 4 ${ }^{\circ} \mathrm{C} / \mathrm{min}$.

The less pure specimens were weighed out in approximately $1 \mathrm{~g}$ batches and mixed with an alumina mortar and pestle. In order to minimize any possible loss of $\mathrm{WO}_{3}$ these specimens were not calcined after mixing.

Subsolidus, as well as melting point data, were obtained by the quenching technique on samples sealed in platinum tubes. An electrically heated vertical tube furnace wound with 80 percent Pt-20 percent Rh wire was used for the quenching experiments. The furnace was controlled by an a-c Wheatstone bridge controller which was capable of holding the temperature to at least $\pm 2{ }^{\circ} \mathrm{C}$ for an extended period of time. Temperatures were measured with a $\mathrm{Pt}$ versus Pt-10 percent $\mathrm{Rh}$ thermocouple which had been calibrated against the melting points of $\mathrm{Au}\left(1063{ }^{\circ} \mathrm{C}\right)$ and $\mathrm{Pd}\left(1552{ }^{\circ} \mathrm{C}\right)[12]$. The thermocouple was recalibrated several times during the course of the work. Specimens were suspended in the furnace by fine $\mathrm{Pt}$ wire. In order to quench the wire was fused allowing the sealed tubes to drop out of the heating chamber into a beaker of water. When the tubes were opened the specimens were examined for physical appearances of melting. The first sign of glazing of the surface of the specimen was interpreted as the first experimental evidence for the solidus temperature. Acceptance of this appearance as evidence of melting was found justified in many specimens by an abrupt difference in the x-ray diffraction powder patterns of the specimens. The formation of a concave meniscus, without the formation of relatively large crystals, indicated the liquidus temperature. The overall reproducibility of the temperature measurements for the experimental data points was within $\pm 2{ }^{\circ} \mathrm{C}$ or better and the overall accuracy of the reported temperatures was within $\pm 5{ }^{\circ} \mathrm{C}$ or better.

Equilibrium was considered to have been obtained when the $x$-ray diffraction patterns of specimens successively heated for longer times and/or at higher temperatures showed no change. X-ray diffraction powder patterns were made using a high-angle recording Geiger counter diffractometer and nickel-filtered copper radiation, with the Geiger counter traversing the specimen at $1 / 4^{\circ} 2 \theta / \mathrm{min}$ and the intensity of the radiation being recorded on the chart at $1^{\circ} 2 \theta /$ in. The unit cell dimensions reported can be considered accurate to about \pm 2 in the last decimal place listed.

\section{Compounds in the $\mathrm{Nb}_{2} \mathrm{O}_{5}-\mathrm{WO}_{3}$ System}

\section{1. $\mathrm{Nb}_{2} \mathrm{O}_{5}$}

The stability relations of the various reported polymorphs of $\mathrm{Nb}_{2} \mathrm{O}_{5}$ have been summarized by several workers [13-17]. It has been concluded that the hightemperature monoclinic form of $\mathrm{Nb}_{2} \mathrm{O}_{5}$ is the only 
stable form at atmospheric pressure $[14,16,17]$. The crystal structure of the high-temperature modification of $\mathrm{Nb}_{2} \mathrm{O}_{5}$ has been described by Gatehouse and Wadsley [18]. They report this phase to be monoclinic, (space group P2) with 14 formula units in the unit cell and $a=21.16 \AA, b=3.822 \AA, c=19.35 \AA$, $\beta=119^{\circ} 50^{\prime}$. The x-ray diffraction powder pattern previously listed for $\mathrm{Nb}_{2} \mathrm{O}_{5}$ [19] can be more correctly indexed on the basis of the published single-crystal intensity data and is shown in table 1 . This indexing leads to slightly revised values of the unit cell dimensions, as follows: $a=21.149 \AA, b=3.823 \AA, c=19.352$ $\AA, \beta=119^{\circ} 48^{\prime}$, in excellent agreement with the lattice constants given by Gatehouse and $\mathrm{W}$ adsley [18]. The melting point of the pure $\mathrm{Nb}_{2} \mathrm{O}_{5}$ used for this study was previously determined to be $1485{ }^{\circ} \mathrm{C}[20]$.

TABLE 1. X-ray diffraction powder data for $\mathrm{Nb}_{2} \mathrm{O}_{5}($ CuK a radiation $)$

\begin{tabular}{|c|c|c|c|c|c|c|c|c|c|}
\hline$d^{\mathrm{a}}$ & $I^{\mathrm{b}}$ & $\frac{1}{d^{2}}$ obs & $\frac{1}{d^{2}}$ calc & $h k l^{\mathrm{c}}$ & $d^{\mathrm{a}}$ & $I^{\mathrm{b}}$ & $\frac{1}{d^{2}}$ obs & $\frac{1}{d^{2}}$ calc & $h k l^{c}$ \\
\hline $\begin{array}{l}16.66 \\
10.517 \\
10.063 \\
9.615 \\
9.148\end{array}$ & $\begin{array}{l}6 \\
5 \\
2 \\
2 \\
5\end{array}$ & $\begin{array}{r}0.0036 \\
.0090 \\
.0099 \\
.0108 \\
.0120\end{array}$ & $\begin{array}{r}0.0036 \\
.0090 \\
.0097 \\
.0107 \\
.0118\end{array}$ & $\begin{array}{l}001 \\
201 \\
101 \\
102 \\
200\end{array}$ & $\begin{array}{l}2.523 \\
2.491\end{array}$ & $\begin{array}{r}5 \\
26\end{array}$ & $\begin{array}{l}.1571 \\
.1612\end{array}$ & $\left\{\begin{array}{r}0.1526 \\
.1541 \\
.1543 \\
.1571 \\
.1612\end{array}\right.$ & $\begin{array}{l}80 \overline{2} \\
10 \overline{7} \\
61 \overline{4} \\
015 \\
70 \overline{7}\end{array}$ \\
\hline $\begin{array}{l}8.354 \\
6.942 \\
6.486 \\
6.285 \\
5.590\end{array}$ & $\begin{array}{r}4 \\
2 \\
3 \\
11 \\
4\end{array}$ & $\begin{array}{l}.0143 \\
.0208 \\
.0238 \\
.0253 \\
.0320\end{array}$ & $\begin{array}{l}.0142 \\
.0206 \\
.0236 \\
.0252 \\
.0319\end{array}$ & $\begin{array}{l}002 \\
301 \\
102 \\
10 \overline{3} \\
003\end{array}$ & 2.452 & 5 & .1664 & $\left\{\begin{array}{l}.1623 \\
.1625 \\
.1662 \\
.1672 \\
.1865\end{array}\right.$ & $\begin{array}{l}511 \\
214 \\
41 \frac{1}{6} \\
615 \\
413\end{array}$ \\
\hline $\begin{array}{l}5.273 \\
5.116\end{array}$ & $\begin{array}{r}6 \\
48\end{array}$ & $\begin{array}{l}.0360 \\
.0382\end{array}$ & $\begin{array}{l}.0359 \\
.0382\end{array}$ & $\begin{array}{l}40 \overline{2} \\
40 \overline{1}\end{array}$ & 2.314 & 29 & .1865 & $\left\{\begin{array}{l}.1869 \\
.1872\end{array}\right.$ & $\begin{array}{l}61 \overline{6} \\
20 \overline{8}\end{array}$ \\
\hline $\begin{array}{l}4.734 \\
4.616 \\
3.852\end{array}$ & $\begin{array}{r}4 \\
36 \\
6\end{array}$ & $\begin{array}{l}.0446 \\
.0469 \\
.0674\end{array}$ & $\begin{array}{l}.0446 \\
.0465 \\
.0670\end{array}$ & $\begin{array}{l}103 \\
104 \\
305\end{array}$ & $\begin{array}{l}2.114 \\
2.076 \\
2.037\end{array}$ & $\begin{array}{r}4 \\
38 \\
34\end{array}$ & $\begin{array}{l}.2244 \\
.2321 \\
.2411\end{array}$ & $\begin{array}{l}.2239 \\
.2321 \\
.2410\end{array}$ & $\begin{array}{r}414 \\
10, \frac{4}{3} \\
209\end{array}$ \\
\hline & & & & & 1.912 & 29 & .2736 & .2736 & 020 \\
\hline 3.821 & 6 & .0685. & $\begin{array}{l}.0683 \\
.0684\end{array}$ & $\begin{array}{l}205 \\
010\end{array}$ & & & & 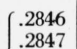 & $\begin{array}{l}3,0,1 \overline{0} \\
11,0, \overline{7}\end{array}$ \\
\hline $\begin{array}{l}3.737 \\
3.636\end{array}$ & $\begin{array}{l}100 \\
100\end{array}$ & .0716 & $\begin{array}{l}.0714 \\
.0717\end{array}$ & $\begin{array}{l}110 \\
11 \frac{1}{1}\end{array}$ & 1.873 & 5 & .2852 & $\left\{\begin{array}{l}.2847 \\
.2855\end{array}\right.$ & $\begin{array}{r}11,0, \overline{3} \\
220\end{array}$ \\
\hline 3.636 & 100 & .0756. & .0755 & 105 & 1.856 & 3 & .2903 & .2904 & \\
\hline 3.553 & 4 & .0792 & .0791 & $11 \overline{2}$ & 1.819 & 20 & .3020 & $\left\{\begin{array}{l}.3020 \\
3031\end{array}\right.$ & $\begin{array}{l}2,0,1 \overline{0} \\
11,0, \overline{8}\end{array}$ \\
\hline 3.515 & 4 & .0809 & .0807 & $60 \overline{3}$ & & & & .3115 & 407 \\
\hline 3.483 & 100 & .0824 & $\begin{array}{l}.0824 \\
.0826\end{array}$ & $\begin{array}{l}602 \\
012\end{array}$ & 1.789 & 16 & .3124 & $\left\{\begin{array}{l}.3118 \\
.3127\end{array}\right.$ & $\begin{array}{l}421 \\
902\end{array}$ \\
\hline $\begin{array}{l}3.406 \\
3.383\end{array}$ & $\begin{array}{l}4 \\
4\end{array}$ & $\begin{array}{l}.0862 \\
.0877\end{array}$ & $\begin{array}{l}.0859 \\
.0877\end{array}$ & $\begin{array}{l}60 \overline{4} \\
303\end{array}$ & & & & .3201 & \\
\hline 3.351 & 28 & .0891. & $\begin{array}{l}.0886 \\
.0890\end{array}$ & $\begin{array}{l}005 \\
31 \overline{1}\end{array}$ & 1.765 & 5 & .3211 & $\begin{array}{l}.3227 \\
.3230\end{array}$ & $12,0, \frac{5}{6}$ \\
\hline $\begin{array}{l}3.316 \\
3.264\end{array}$ & $\begin{array}{l}5 \\
4\end{array}$ & $\begin{array}{l}.0909 \\
.0939\end{array}$ & $\begin{array}{l}.0911 \\
.0936\end{array}$ & $60 \frac{1}{3}$ & 1.742 & 23 & .3295. & $\begin{array}{l}.3231 \\
.3292 \\
.3296\end{array}$ & $\begin{array}{r}6,0,11 \\
119 \\
12,0, \frac{9}{4}\end{array}$ \\
\hline $\begin{array}{l}3.247 \\
3.153 \\
3.078\end{array}$ & $\begin{array}{r}4 \\
11 \\
6\end{array}$ & $\begin{array}{l}.0949 \\
.1006 \\
.1055\end{array}$ & $\begin{array}{l}.0951 \\
.1003 \\
.1051\end{array}$ & $\begin{array}{l}310 \\
013 \\
506\end{array}$ & 1.727 & 25 & .3353 & $\begin{array}{r}.3353 \\
\int .3422\end{array}$ & $\begin{array}{l}8,0, \overline{1} \\
11,1, \overline{4}\end{array}$ \\
\hline $\begin{array}{l}2.994 \\
2.832\end{array}$ & $\begin{array}{r}7 \\
36\end{array}$ & $\begin{array}{l}.1116 \\
.1247\end{array}$ & $\begin{array}{l}.1113 \\
.1246\end{array}$ & $\begin{array}{l}10 \overline{6} \\
51 \overline{2}\end{array}$ & $\begin{array}{l}1.709 \\
1.692\end{array}$ & $\begin{array}{l}10 \\
20\end{array}$ & $\begin{array}{l}.3424 \\
.3493\end{array}$ & $\begin{array}{l}.3422 \\
.3425 \\
.3491 \\
3530\end{array}$ & $\begin{array}{l}11,1, \frac{4}{6} \\
12 \frac{5}{5}\end{array}$ \\
\hline 2.826 & 38 & .1252 & $\begin{array}{l}.1248 \\
.1262\end{array}$ & $\begin{array}{l}014 \frac{4}{3} \\
513\end{array}$ & 1.683 & 51 & .3529 & $\left\{\begin{array}{l}.3530 \\
.3531\end{array}\right.$ & $\begin{array}{l}3,1,1 \overline{0} \\
11,1, \overline{3}\end{array}$ \\
\hline 2.771 & 31 & 1303 & .1301 & $5 \overline{11}$ & $\begin{array}{l}1.627 \\
1.592\end{array}$ & $\begin{array}{r}8 \\
27\end{array}$ & $\begin{array}{l}.3776 \\
.3945\end{array}$ & $\begin{array}{l}.3776 \\
.3946\end{array}$ & $\begin{array}{r}408 \\
7,1,11\end{array}$ \\
\hline 2.701 & 34 & (1371. & $\begin{array}{l}.1367 \\
.1377\end{array}$ & $\begin{array}{l}215 \\
706 \\
\end{array}$ & 1.582 & 20 & .3994 & $\left\{\begin{array}{l}.3982 \\
3995\end{array}\right.$ & $\begin{array}{r}11,0,1 \\
318\end{array}$ \\
\hline 2.668 & 3 & 列. 1405 & $\begin{array}{l}.1401 \\
.1405 \\
.1426\end{array}$ & $\begin{array}{l}41 \overline{5} \\
20 \overline{7}\end{array}$ & $\begin{array}{l}1.579 \\
1.556\end{array}$ & $\begin{array}{l}18 \\
22\end{array}$ & $\begin{array}{l}.4012 \\
.4130\end{array}$ & $\begin{array}{l}.4011 \\
.4133\end{array}$ & $\begin{array}{r}10,1, \frac{1}{12} \\
12,1, \frac{8}{1}\end{array}$ \\
\hline 2.644 & 4 & . 1431. & $\begin{array}{l}.1426 \\
.1433\end{array}$ & $\begin{array}{l}510 \\
80 \underline{4}\end{array}$ & & & & & \\
\hline 2.628 & 5 & . 1448. & $\begin{array}{l}.1445 \\
.1452\end{array}$ & $\begin{array}{l}80 \overline{3} \\
60 \overline{\overline{7}}\end{array}$ & & & & & \\
\hline
\end{tabular}

anterplaner spacing.

b Intensity relative to the strongest peak(s).

c Indexed on the basis of a monoclinic unit cell (space group $\mathrm{P}_{2}$ ) $a=21.149 \AA, b=3.823 \AA$, $c=19.352 \AA, \beta=119^{\circ} 48^{\prime}$ and the previously reported observed structure factors from the single crystal data [18].

\subsection{Compounds Structurally Related to $\mathrm{Nb}_{2} \mathrm{O}_{5}$}

a. Compounds Belonging to the Homologous Series $\mathbf{B}_{n m+1} \mathbf{O}_{3 n m-(n+m)+4}$

Roth and Wadsley [4] have shown that most of the phases formed by the addition to $\mathrm{Nb}_{2} \mathrm{O}_{5}$ of oxides with cations similar in size to $\mathrm{Nb}^{+5}$, regardless of valence, can be described by the homologous series notation $\mathrm{B}_{n m p+1} \mathrm{O}_{3 n m p-(n+m) p+4}$. Structurally, these phases are made up of blocks of octahedra in a given plane $n$ long and $m$ wide with a variable number $(p)$ of blocks connected by edge sharing of octahedra at the corners of the blocks. Similar block units occur at two levels in the unit cell, zero and one-half, in a

TABLE 2. X-ray diffraction powder data for the compound $\mathrm{WNb}_{12} \mathrm{O}_{33}$ $\left(\mathrm{CuK} \mathrm{K}_{a}\right.$ radiation)

\begin{tabular}{|c|c|c|c|c|c|c|c|c|c|}
\hline $\begin{array}{l}d^{\text {a }} \\
\text { obs }\end{array}$ & $\begin{array}{l}I^{\mathrm{b}} \\
\text { obs }\end{array}$ & $\frac{1}{d^{2}}$ obs & $\frac{1}{d^{2}}$ calc & $h k l^{\mathrm{c}}$ & $\begin{array}{l}d^{\text {a }} \\
\text { obs }\end{array}$ & $\begin{array}{l}I^{\mathrm{b}} \\
\mathrm{obs}\end{array}$ & $\frac{1}{d^{2}}$ obs & $\frac{1}{d^{2}}$ calc & $h k l^{\mathrm{c}}$ \\
\hline 14.77 & 19 & 0.0046 & 0.0046 & 001 & 2.167 & 6 & 0.2130 & 0.2135 & $10.0, \overline{6}$ \\
\hline 11.08 & 23 & .0081 & .0081 & $20 \overline{1}$ & 228 & 5 & 2200 & $\{.2213$ & 801 \\
\hline $\begin{array}{l}7.42 \\
6.43\end{array}$ & $\begin{array}{r}16 \\
8\end{array}$ & $\begin{array}{l}.0182 \\
0242\end{array}$ & $\begin{array}{r}.0183 \\
0241\end{array}$ & $\begin{array}{l}002 \\
201\end{array}$ & 2.128 & 3 & .2209 & $\{.2213$ & \\
\hline $\begin{array}{l}6.43 \\
5.54\end{array}$ & 18 & . .0326 & . .0325 & $40 \overline{2}$ & 2.116 & 6 & .2233 & $\left\{\begin{array}{l}.2236 \\
.2237\end{array}\right.$ & $\begin{array}{l}0 \\
20\end{array}$ \\
\hline 5.35 & 6 & .0349 & .0348 & $40 \overline{\underline{1}}$ & 2.112 & 6 & .2242 & .2244 & \\
\hline 5.04 & 48 & .0394 & .0394 & 403 & 2.072 & 67 & .2325 & .2328 & $10,0, \overline{7}$ \\
\hline 4.937 & 12 & . 0410 & . 04111 & 003 & & & & & \\
\hline 4.674 & 58 & . 0458 & .0458 & 202 & 2.042 & 52 & 2398 & .2401 & 4 \\
\hline 3.743 & 76 & . 0714 & .0713 & 110 & 2.006 & 6 & .2484 & .2487 & 912 \\
\hline 3.729 & 96 & .0719 & .0718 & $11 \overline{1}$ & 1.9375 & 9 & 2664 & $\left\{\begin{array}{l}.2669 \\
2669\end{array}\right.$ & \\
\hline 3.702 & 40 & 年. & .0730 & 004 & & & & & \\
\hline 3.697 & 40 & (0732 & (0732 & $60 \overline{3}$ & 1.9324 & 8 & 2678 & .2683 & 31 \\
\hline 3.670 & 20 & . 0742 & . 0742 & $60 \overline{2}$ & 1.9121 & 60 & .2735 & .2735 & 02 \\
\hline 3.616 & 284 & 等. .75 & 等. 7666 & 203 & 18729 & 4 & 2851 & 2851 & \\
\hline 3.535 & 43 & .0800 & .0798 & 111 & & 5 & 2871 & & \\
\hline 3.510 & 300 & . 0812 & .0811 & $60 \underline{4}$ & 1.8560 & 15 & .2899 & .2903 & $12,0,5$ \\
\hline 3.437 & 13 & . 0847 & . 0846 & $60 \overline{\overline{1}}$ & & & & .2910 & \\
\hline 3.415 & 15 & . 0857 & . 0857 & $20 \overline{\overline{5}}$ & 1.8524 & 29 & 2914 & $\{.2918$ & \\
\hline 3.358 & 29 & . 0887 & . 0887 & $31 \overline{2}$ & & & & .2920 & \\
\hline 3.203 & 27 & .0974 & . 09 & 11 & 1.8342 & 8 & 2969 & .2972 & $12,0, \overline{4}$ \\
\hline 2.930 & 16 & .1165 & .1165 & 204 & 1.8144 & 8 & .3038 & .3040 & $12,0, \overline{7}$ \\
\hline & 5 & . 1189 & .1189 & 51 & 1.80 & 20 & .3056 & .3063 & \\
\hline 2.866 & 58 & .1217 & 1218 & $51 \overline{3}$ & 1.80 & 8 & . 3084 & .3093 & \\
\hline 2.836 & 37 & .1243 & . 1243 & 113 & 1.7895 & 13 & .3126 & .3123 & $11,1, \overline{5}$ \\
\hline 2.799 & 7 & 1277 & . 1279 & $2 \overline{6}$ & & 8 & .3185 & .3185 & $11,1,6$ \\
\hline 2.735 & 41 & .1337 & .1337 & $51 \overline{4}$ & 1.7697 & 9 & .3193 & .3193 & \\
\hline 2.706 & 31 & .1365 & 列. 1366 & 312 & 1.76 & 22 & .3214 & .3217 & 8,0 , \\
\hline & & & $\int .1392$ & $80 \overline{2}$ & 1.75 & 19 & .3240 & .3245 & 12,0 \\
\hline 2.682 & 17 & . 1390 & $\{.1392$ & $80 \overline{\overline{5}}$ & $\begin{array}{l}1.7403 \\
1.7329\end{array}$ & $\begin{array}{l}21 \\
19\end{array}$ & $\begin{array}{l}.3302 \\
3330\end{array}$ & $\begin{array}{l}.3305 \\
3332\end{array}$ & 10,0 \\
\hline 2.542 & 8 & .1548 & .1548 & $51 \overline{5}$ & & & & & \\
\hline & & & & & 1.7311 & 18 & .3337 & .3339 & $11,1, \overline{7}$ \\
\hline 2.520 & 116 & .1575 & $\left\{\begin{array}{l}.1574 \\
1580\end{array}\right.$ & $\begin{array}{l}801 \\
40 \overline{7}\end{array}$ & 1.6903 & 20 & .3500 & .3501 & \\
\hline & & & 159 & & 1.6805 & 42 & .3541 & $\left\{\begin{array}{l}3545 \\
3547\end{array}\right.$ & $\begin{array}{l}515 \\
62 \overline{4}\end{array}$ \\
\hline 2.499 & 10 & . 1602 & $\left\{\begin{array}{l}.1603 \\
\text { S }\end{array}\right.$ & 114 & 1.6709 & 26 & .3582 & .3585 & 11,1, \\
\hline & 16 & & & & 1.6088 & 21 & .3864 & .3869 & 10,0 \\
\hline & 4 & .1750 & $\begin{array}{l}.1651 \\
.1722\end{array}$ & $51 \frac{1}{2}$ & & & & & \\
\hline 2.339 & 6 & .1828 & .1831 & 404 & $\begin{array}{l}1.6070 \\
1.5975\end{array}$ & $\begin{array}{l}23 \\
16\end{array}$ & $\begin{array}{l}.3872 \\
.3918\end{array}$ & $\begin{array}{l}.3880 \\
.3924\end{array}$ & \\
\hline & & & 1842 & & 1.5838 & 21 & .3987 & .3992 & $9,1,10$ \\
\hline 2.328 & 15 & . 1845 & $\left\{\begin{array}{l}1042 \\
1848\end{array}\right.$ & 715 & 1500 & (20 & 4005 & $\int 4006$ & $14,0, \overline{5}$ \\
\hline & & & & 800 & 1.5801 & 20 & .4005 & $\{.4017\}$ & $10,0, \overline{11}$ \\
\hline 2.316 & 29 & 1864 & $\left\{\begin{array}{l}.1865 \\
.1965\end{array}\right.$ & $711 \overline{1}$ & 1.5734 & 21 & .4039 & .4049 & 119 \\
\hline & & & 0.1868 & 316 & 1.56 & 19 & .4105 & .41 & \\
\hline $\begin{array}{l}2.215 \\
2.183\end{array}$ & 11 & $\begin{array}{l}.2038 \\
.2000\end{array}$ & . 2043 & $60 \overline{8}$ & 1.55 & 9 & .4148 & & \\
\hline & 7 & .2098 & 2099 & 710 & 1.5405 & 5 & .4214 & .4227 & $4,0,1$ \\
\hline
\end{tabular}

a Interplanar spacing.

Observed intensity. $c=17.724 \AA, \beta=123^{\circ} 22^{\prime}$ and the previously reported structure factors from the single crystal data [2]. 
plane perpendicular to one axis which always has a value of approximately $3.8 \AA$. This axis is the $b$-axis of a monoclinic cell or the $c$-axis of a tetragonal cell. The octahedra occurring at the two different levels are connected to each other by edge sharing except at the junction of four such blocks, two at each level, where they are bonded by a tetrahedrally coordinated cation. Those compounds in the $\mathrm{Nb}_{2} \mathrm{O}_{5}-\mathrm{WO}_{3}$ system whose structures have been described all have $p=1$ and the formula simplifies to $\mathrm{B}_{n m+1} \mathrm{O}_{3 n m-(n+m)+4}$, where the tetrahedral position is always occupied by $\mathrm{W}^{+6}$ ions. In this arrangement when $n=m$, the unit cell is body-centered tetragonal as in $\mathrm{PNb}_{9} \mathrm{O}_{25}$ [21]. When $n \neq m$ the cell is C-centered monoclinic.

\section{b. $6 \mathrm{Nb}_{2} \mathrm{O}_{5} \cdot \mathrm{WO}_{3}, \mathrm{WNb}_{12} \mathrm{O}_{33}$ )}

The unit cell of this compound is C-centered monoclinic (probable space group $\mathrm{C}_{2}$ ) having $n=3, m=4$ [2]. The indexed $x$-ray diffraction powder pattern obtained in the present study is listed in table 2. The unit cell dimensions derived from this pattern are $a=22.282$ $\AA, b=3.824 \AA, c=17.724 \AA, \beta=123^{\circ} 22^{\prime}$. $W^{\prime} \mathrm{Wb}_{12} \mathrm{O}_{33}$ is apparently stable from room temperature to the congruent melting point at $1476{ }^{\circ} \mathrm{C}$.

\section{c. $7 \mathrm{Nb}_{2} \mathrm{O}_{5} \cdot 3 \mathrm{WO}_{3}\left(\mathrm{~W}_{3} \mathrm{Nb}_{14} \mathrm{O}_{44}\right)$}

The unit cell of this compound is body-centered tetragonal, space group $\mathrm{I} 4 / \mathrm{m}$ or $\mathrm{I} \overline{4}(n=4, m=4[3])$. The indexed x-ray diffraction powder pattern is listed in table 3 and the unit cell dimensions derived from this pattern are $a=21.002 \AA, c=3.820 \AA$.

\section{d. $8 \mathrm{Nb}_{2} \mathrm{O}_{5} \cdot 5 \mathrm{WO}_{3}\left(\mathrm{~W}_{5} \mathrm{Nb}_{16} \mathrm{O}_{55}\right)$}

The unit cell of this phase is C-centered monoclinic space group $\mathrm{C}_{2}(n=4, m=5,[2])$. The indexed x-ray diffraction powder pattern is listed in table 4 and the unit cell dimensions derived from this pattern are $a=29.638 \AA, b=3.820 \AA, c=23.126 \AA, \beta=126^{\circ} 27^{\prime}$.
TABLE 4. X-ray diffraction powder data for the compound $\mathrm{W}_{5} \mathrm{Nb}_{16} \mathrm{O}_{55}$ (CuKa radiation)

\begin{tabular}{|c|c|c|c|c|c|c|c|c|c|}
\hline$d^{\mathrm{a}}$ & $I^{\mathrm{b}}$ & $\frac{1}{d^{2}}$ obs & $\frac{1}{d^{2}}$ calc & $h k l^{\mathrm{c}}$ & $d^{\mathrm{a}}$ & $I^{\mathrm{b}}$ & $\frac{1}{d^{2}}$ obs & $\frac{1}{d^{2}}$ calc & $h k l^{c}$ \\
\hline 18.58 & 7 & 0.0029 & 0.0029 & 001 & 2.397 & 4 & 0.1741 & 0.1744 & $91 \overline{2}$ \\
\hline 14.84 & 12 & .0045 & .0046 & $20 \overline{\mathrm{l}}$ & 2.382 & 8 & .1763 & .1764 & 711 \\
\hline 9.31 & 13 & .0115 & .0116 & 002 & 2.350 & 4 & .1811 & .1811 & $12,0, \overline{8}$ \\
\hline 7.40 & 17 & .0183 & .0183 & $40 \overline{2}$ & 2.316 & \multirow[t]{2}{*}{7} & \multirow{2}{*}{.1864} & $\{.1862$ & 207 \\
\hline 6.75 & 4 & .0220 & .0220 & $40 \overline{3}$ & \multirow{3}{*}{2.294} & & & .1873 & $8,0, \overline{10}$ \\
\hline 6.21 & 12 & .0260 & .0260 & 003 & & 37 & .1900 & & $91 \overline{1}$ \\
\hline 5.85 & 6 & .0293 & .0293 & 202 & & & & & 518 \\
\hline 4.935 & 12 & .0411 & .0411 & $60 \underline{\overline{3}}$ & \multirow{2}{*}{2.254} & \multirow{2}{*}{6} & \multirow{2}{*}{.1969} & $\int .1968$ & \multirow{2}{*}{$\begin{array}{r}315 \\
10,0,10\end{array}$} \\
\hline 4.701 & 45 & .0453 & .0453 & $60 \overline{4}$ & & & & \multirow{5}{*}{$\begin{array}{l}. .1970 \\
.2111 \\
.2260 \\
.2340 \\
.2413\end{array}$} & \\
\hline 4.652 & 10 & .0462 & .0462 & 004 & \multirow{3}{*}{$\begin{array}{l}2.177 \\
2.104 \\
2.067\end{array}$} & \multirow{2}{*}{$\begin{array}{l}6 \\
8\end{array}$} & \multirow{4}{*}{$\begin{array}{l}.2111 \\
.2260 \\
.2340 \\
.2413\end{array}$} & & \multirow{2}{*}{$\begin{array}{r}910 \\
519\end{array}$} \\
\hline 4.512 & 35 & .0491 & .0491 & 203 & & & & & \\
\hline 3.771 & 130 & .0703 & .07 & 110 & & $\begin{array}{r}8 \\
52\end{array}$ & & & $\begin{array}{l}11,1,2 \\
14,0,9\end{array}$ \\
\hline 3.767 & 142 & .0705 & .07 & $11 \overline{1}$ & & \multirow[b]{2}{*}{36} & & & \multirow{2}{*}{$\begin{array}{r}14,0,9 \\
407\end{array}$} \\
\hline 3.717 & 26 & .0724 & .0722 & $00 \underline{5}$ & & & \multirow{2}{*}{$\begin{array}{l}.2448 \\
.2642\end{array}$} & .2448 & \\
\hline 3.705 & 22 & .0729 & .0731 & $80 \overline{4}$ & \multirow{4}{*}{$\begin{array}{l}1.9453 \\
1.9099 \\
1.8827 \\
1.8783\end{array}$} & \multirow{2}{*}{$\begin{array}{r}4 \\
37\end{array}$} & & \multirow{2}{*}{$\begin{array}{l}.2638 \\
.2742\end{array}$} & 606 \\
\hline 3.660 & 250 & .0747 & $\{.0743$ & $80 \overline{3}$ & & & \multirow{3}{*}{$\begin{array}{l}.2642 \\
.2742 \\
.2821 \\
.2835\end{array}$} & & 020 \\
\hline & & .0147 & $\{.0747$ & 204 & & 6 & & .2823 & $10,0,3$ \\
\hline 3.630 & 40 & .0759 & .0759 & 111 & & 6 & & .2837 & $12,0, \overline{12}$ \\
\hline 3.587 & 222 & .0777 & .0777 & $80 \overline{5}$ & 1.8602 & 17 & .2890 & .2890 & $0.0,10$ \\
\hline 3.539 & 25 & .0798 & $\begin{array}{r}.0799 \\
\end{array}$ & 312 & 1.8503 & 7 & .2921 & .2919 & $16,0, \overline{7}$ \\
\hline & 8 & & .0813 & 802 & 1.8329 & 18 & .2977 & $\int .2972$ & $16,0,6$ \\
\hline 3.406 & 8 & .0862 & .0863 & 403 & 1.8294 & 26 & .2988 & $\begin{array}{r}.2978 \\
.2988\end{array}$ & $2,0,11$ \\
\hline 3.386 & 10 & .0872 & .08 & 112 & $\begin{array}{l}1.8294 \\
1.8007\end{array}$ & 12 & $\begin{array}{l}.2988 \\
.3084\end{array}$ & $\begin{array}{l}.2988 \\
.3083\end{array}$ & $\begin{array}{r}408 \\
160 \frac{5}{6}\end{array}$ \\
\hline 3.252 & 6 & .0946 & .0947 & $40 \overline{\overline{7}}$ & & & & & $16,0,5$ \\
\hline 3.188 & 16 & .0984 & .0984 & $51 \overline{3}$ & 1.7944 & 18 & .3106 & .3107 & $16,0, \overline{10}$ \\
\hline & 12 & .1044 & .1044 & 113 & 1.7800 & 4 & .3156 & $\{.3153$ & $62 \overline{3}$ \\
\hline 3.072 & 10 & .1060 & .1061 & 205 & 1.7691 & 7 & .3195 & $\begin{array}{r}.3157 \\
3195\end{array}$ & $4,0,12$ \\
\hline 2.980 & 7 & .1126 & .1126 & 800 & & & & $\begin{array}{r}.3195 \\
3233\end{array}$ & 624 \\
\hline 2.897 & 8 & .1192 & .1192 & $10,0, \overline{6}$ & 1.7580 & 25 & .3236 & $\left\{\begin{array}{l}.3233 \\
.3238\end{array}\right.$ & $\begin{array}{r}223 \\
120\end{array}$ \\
\hline $\begin{array}{l}2.832 \\
2.817\end{array}$ & $\begin{array}{l}16 \\
47\end{array}$ & $\begin{array}{l}.1246 \\
.1260\end{array}$ & $\begin{array}{l}.1245 \\
.1260\end{array}$ & $\begin{array}{l}71 \overline{3} \\
71 \overline{4}\end{array}$ & 1.7452 & 8 & .3283 & $\begin{array}{l}.0280 \\
.3285\end{array}$ & $\begin{array}{r}12,15 \\
119\end{array}$ \\
\hline 2.804 & 40 & .1272 & .1273 & 114 & 1.7421 & 14 & .3295 & .3292 & $12,0,2$ \\
\hline & & & (.1274 & & 1.7311 & 24 & .333 & & 318 \\
\hline $\begin{array}{l}2.775 \\
2.740\end{array}$ & $\begin{array}{r}5 \\
54\end{array}$ & .1299 & .1300 & $10,0, \overline{\overline{7}}$ & 1.7229 & 12 & .3369 & .3368 & $15,1, \overline{9}$ \\
\hline $\begin{array}{l}2.740 \\
2.732\end{array}$ & 54 & .1332 & .1332 & 715 & 1.6943 & 28 & 3484 & $\int .3479$ & 517 \\
\hline $\begin{array}{l}2.732 \\
2.727\end{array}$ & $\begin{array}{l}65 \\
71\end{array}$ & .1340 & .1339 & $10,0, \overline{2}$ & & & & $\{.3489$ & 224 \\
\hline & 71 & .1345 & .1345 & 313 & 1.6862 & 57 & .3517 & $\left\{\begin{array}{l}.3515 \\
2515\end{array}\right.$ & $15,1,1 \underline{0}$ \\
\hline $\begin{array}{l}2.615 \\
2.574\end{array}$ & 8 & .1462 & .1463 & $71 \overline{6}$ & & & & & 825 \\
\hline 2.574 & 16 & .1509 & .15 & 512 & 1.6303 & 12 & .3763 & .3762 & $12,0,3$ \\
\hline 2.562 & 84 & .1524 & .15 & $10,0, \overline{1}$ & 1.6284 & 13 & .3771 & .3772 & $4,0,13$ \\
\hline & & & & 609 & 1.6101 & 10 & .3857 & .3861 & $14,0, \overline{14}$ \\
\hline 2.549 & 22 & .1539 & $\begin{array}{l}.1538 \\
.1540\end{array}$ & $\begin{array}{l}809 \\
405\end{array}$ & 1.5888 & 12 & .3962 & .3958 & $11,1, \frac{3}{2}$ \\
\hline 2.533 & 8 & .1558 & .1559 & 115 & 1.5783 & 14 & .4014 & $\left\{\begin{array}{l}.4015 \\
.4015\end{array}\right.$ & $\begin{array}{r}13,1,3 \\
428\end{array}$ \\
\hline 2.479 & 6 & .1627 & $\left\{\begin{array}{l}.1627 \\
.1628\end{array}\right.$ & $\begin{array}{l}915 \\
314\end{array}$ & 1.5732 & 26 & .4041 & .4041 & $3,1,12$ \\
\hline 2.425 & 11 & .1701 & .1699 & $12,0, \overline{\overline{7}}$ & 1.5654 & 30 & .4081 & & $17,1, \frac{6}{2}$ \\
\hline & & & 0.1704 & $91 \overline{6}$ & & & & .4081 & $10,2,2$ \\
\hline
\end{tabular}

a Interplaner spacing.
b Observed intensity.

c Indexed on the basis of a monoclinic unit cell (space group $\mathrm{C}_{2}$ ) $a=29.638 \AA, b=3.820 \AA$ $c=23.126 \AA, \beta=126^{\circ} 27^{\prime}$ and the previously reported observed structure factors from the single erystal data $[2]$.

TABLE 3. X-ray diffraction powder data for the compound $\mathrm{W}_{3} \mathrm{Nb}_{14} \mathrm{O}_{44}\left(\right.$ CuK $K_{\alpha}$ radiation $)$

\begin{tabular}{|c|c|c|c|c|c|c|c|c|c|c|c|c|c|c|}
\hline $\begin{array}{l}d^{a} \\
\text { obs }\end{array}$ & $\begin{array}{l}I^{b} \\
\text { obs }\end{array}$ & $\frac{1}{d^{2}}$ obs & $\frac{1}{d^{2}}$ calc & $h k l^{c}$ & $\begin{array}{l}d^{a} \\
\text { obs }\end{array}$ & $\begin{array}{l}I^{b} \\
\text { obs }\end{array}$ & $\frac{1}{d^{2}}$ obs & $\frac{1}{d^{2}}$ calc & $h k l^{c}$ & $\begin{array}{l}d^{a} \\
\text { obs }\end{array}$ & $\begin{array}{l}I^{b} \\
\text { obs }\end{array}$ & $\frac{1}{d^{2}}$ obs & $\frac{1}{d^{2}}$ calc & $h k l^{c}$ \\
\hline 14.87 & 25 & $0.0045^{\circ}$ & 0.0045 & 110 & 2.560 & 22 & 0.1526 & 0.1524 & 611 & 1.8620 & 6 & 0.2884 & 0.2884 & 941 \\
\hline 10.52 & 5 & .0090 & .0091 & 200 & 2.546 & 122 & .1543 & .1542 & 820 & 1.8564 & 15 & .2902 & .2902 & 880 \\
\hline 7.42 & 33 & .0181 & .0181 & 220 & 2.488 & 10 & .1616 & .1615 & 541 & 1.8419 & 28 & .2948 & .2947 & $11,3,0 / 970$ \\
\hline 6.65 & 8 & .0226 & 0227 & 310 & 2.441 & 7 & 1679 & 1678 & 750 & 1.8367 & 15 & .2964 & .2967 & 312 \\
\hline 4.951 & 23 & .0408 & .0408 & 330 & 2.421 & 16 & .1706 & .1705 & 631 & 1.8007 & 54 & .3084 & .3083 & $10,6,0$ \\
\hline 4.694 & 82 & .0454 & .0453 & 420 & 2.358 & 9 & .1799 & .1796 & 701 & 1.7694 & 16 & .3194 & .3194 & 422 \\
\hline 4.118 & 5 & 0590 & 0589 & 510 & 2.347 & 8 & 1814 & .1815 & 840 & 1.7546 & 10 & 3248 & 3247 & 871 \\
\hline 3.757 & 142 & .0708 & .0708 & 101 & 2.319 & 13 & .1860 & .1859 & 910 & 1.7499 & 40 & .3266 & .3265 & $12,0,0$ \\
\hline 3.712 & 37 & .0726 & .0725 & 440 & 2.302 & 41 & .1887 & .1887 & 721 & 1.7305 & 43 & .3339 & .3338 & 961 \\
\hline 3.598 & 480 & .0772 & .0771 & 530 & 2.215 & 2 & .2037 & .2040 & 930 & 1.6874 & 65 & .3512 & .3511 & 532 \\
\hline 3.539 & 52 & .0798 & .0799 & 211 & 2.152 & 9 & .2159 & .2159 & $811 / 741$ & 1.6857 & 85 & .3519 & .3519 & $11,2,1 / 10,5,1$ \\
\hline 3.321 & 20 & .0907 & .0907 & 620 & 2.121 & 3 & .2222 & .2222 & 770 & 1.6612 & 4 & .3624 & .3627 & $12,4,0$ \\
\hline 3.195 & 42 & 0980 & 0980 & 321 & 2.100 & 11 & .2268 & .2267 & $10.0 .0 / 860$ & 1.6559 & 5 & 3647 & 3647 & 622 \\
\hline 2.968 & 8 & .1135 & .1134 & $710 / 550$ & 2.067 & 18 & .2341 & .2340 & 831 & 1.6396 & 5 & .3720 & .3718 & $10,8,0$ \\
\hline 2.912 & 22 & .1179 & .1179 & 640 & 2.039 & 130 & .2405 & .2403 & 950 & 1.6239 & 4 & .3792 & .3791 & $11,4,1$ \\
\hline 2.826 & 80 & .1252 & .1253 & $501 / 431$ & 1.9497 & 3 & .2631 & .2630 & 10.4 .0 & 1.6103 & 35 & .3856 & .3854 & $13,1,0 / 11,7,0$ \\
\hline 2.756 & 21 & .1317 & .1315 & 730 & 1.9102 & 40 & .274 .1 & .2743 & 002 & 1.5861 & 40 & .3975 & .3973 & $12,1,1 / 981$ \\
\hline 2.649 & 105 & .1344 & .1343 & 521 & 1.9004 & 10 & .2769 & .2766 & 11.1 .0 & 1.5686 & 62 & .4064 & .4063 & $10,7.1$ \\
\hline 2.623 & 4 & .1453 & .1451 & 800 & 1.8797 & 5 & .2830 & .2831 & 202 & & & & & \\
\hline
\end{tabular}

${ }^{a}$ Interplaner spacings.

c Indexed on the basis of a tetragonal unit cell (space group $\mathrm{I} 4 / \mathrm{m}$ or $\bar{\Gamma}$ ) $a=21.002 \AA, c=3.820 \AA$. 
TABLE 5. X-ray diffraction powder data for the compound $\mathrm{W}_{8} \mathrm{Nb}_{18} \mathrm{O}_{69}\left(\right.$ CuK $K_{\alpha}$ radiation $)$

\begin{tabular}{|c|c|c|c|c|c|c|c|c|c|c|c|c|c|c|}
\hline$d^{a}$ & $I^{b}$ & $\frac{1}{d^{2}}$ obs & $\frac{1}{d^{2}}$ calc & $h k l^{c}$ & $d^{a}$ & $I^{b}$ & $\frac{1}{d^{2}}$ obs & $\frac{1}{d^{2}}$ calc & $h k^{c}$ & $d^{n}$ & $I^{b}$ & $\frac{1}{d^{2}}$ obs & $\frac{1}{d^{2}}$ calc & $h k l^{c}$ \\
\hline $\begin{array}{l}18.43 \\
9.33 \\
6.20 \\
5.88 \\
4.640\end{array}$ & $\begin{array}{r}4 \\
8 \\
10 \\
10 \\
8\end{array}$ & $\begin{array}{r}0.0029 \\
.0115 \\
.0260 \\
.0290 \\
.0464\end{array}$ & $\begin{array}{r}0.0029 \\
.0116 \\
.0261 \\
.0290 \\
.0464\end{array}$ & $\begin{array}{l}110 \\
220 \\
330 \\
420 \\
440\end{array}$ & $\begin{array}{l}2.731 \\
2.626 \\
2.622 \\
2.575 \\
2.522\end{array}$ & $\begin{array}{r}119 \\
12 \\
18 \\
96 \\
11\end{array}$ & $\begin{array}{l}0.1341 \\
.1450 \\
.1455 \\
.1508 \\
.1573\end{array}$ & $\begin{array}{r}0.1340 \\
.1449 \\
.1456 \\
.1507 \\
.1572\end{array}$ & $\begin{array}{r}631 \\
10,0,0 / 860 \\
721 \\
10,2,0 \\
651\end{array}$ & $\begin{array}{l}1.9340 \\
1.9068 \\
1.8578 \\
1.8482 \\
1.8216\end{array}$ & $\begin{array}{r}4 \\
40 \\
10 \\
12 \\
48\end{array}$ & $\begin{array}{r}0.2674 \\
.2750 \\
.2898 \\
.2928 \\
.3014\end{array}$ & $\begin{array}{r}0.2673 \\
.2750 \\
.2893 \\
.2927 \\
.3014\end{array}$ & $\begin{array}{r}11,4,1 \\
002 \\
14,2,0 / 10,10,0 \\
11,9,0 \\
12,8,0\end{array}$ \\
\hline $\begin{array}{l}4.5 \theta 3 \\
4.375 \\
3.771 \\
3.712 \\
3.642\end{array}$ & $\begin{array}{r}80 \\
5 \\
146 \\
25 \\
372\end{array}$ & $\begin{array}{l}.0493 \\
.0522 \\
.0703 \\
.0726 \\
.0753\end{array}$ & $\begin{array}{l}.0493 \\
.0522 \\
.0702 \\
.0725 \\
.0754\end{array}$ & $\begin{array}{r}530 \\
600 \\
101 \\
710 / 550 \\
640\end{array}$ & $\begin{array}{l}2.477 \\
2.458 \\
2.378 \\
2.304 \\
2.283\end{array}$ & $\begin{array}{r}15 \\
7 \\
9 \\
6 \\
38\end{array}$ & $\begin{array}{l}.1630 \\
.1744 \\
.1769 \\
.1885 \\
.1918\end{array}$ & $\begin{array}{l}.1629 \\
.1745 \\
.1768 \\
.1884 \\
.1919\end{array}$ & $\begin{array}{r}811 / 741 \\
831 \\
11,1,0 \\
11,3,0 / 970 \\
921 / 761\end{array}$ & $\begin{array}{l}1.7791 \\
1.7561 \\
1.7474 \\
1.7384 \\
1.7235\end{array}$ & $\begin{array}{r}12 \\
15 \\
26 \\
7 \\
28\end{array}$ & $\begin{array}{l}.3160 \\
.3243 \\
.3275 \\
.3309 \\
.3366\end{array}$ & $\begin{array}{r}.3159 \\
.3243 \\
.3275 \\
.3310 \\
.3362 \\
3368\end{array}$ & $\begin{array}{r}13,7,0 \\
532 \\
15,1,0 \\
10,9,1 \\
14,6,0\end{array}$ \\
\hline $\begin{array}{l}3.450 \\
3.378 \\
3.185\end{array}$ & $\begin{array}{l}15 \\
17 \\
12\end{array}$ & $\begin{array}{l}.0840 \\
.0876 \\
.0986\end{array}$ & $\begin{array}{l}.0840 \\
.0876 \\
.0985\end{array}$ & $\begin{array}{l}730 \\
321 \\
820\end{array}$ & $\begin{array}{l}2.253 \\
2.248 \\
2.242\end{array}$ & $\begin{array}{r}11 \\
7 \\
6\end{array}$ & $\begin{array}{l}.1970 \\
.1978 \\
.2086\end{array}$ & $\begin{array}{l}.1971 \\
.1977 \\
.2087\end{array}$ & $\begin{array}{r}10,6,0 \\
851 \\
12,0,0\end{array}$ & 1.6943 & 57 & .3484 & .3484 & $12,7,1$ \\
\hline $\begin{array}{l}3.088 \\
3.054\end{array}$ & 15 & $\begin{array}{l}.1049 \\
.1072\end{array}$ & 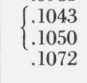 & $\begin{array}{r}660 \\
501 / 431 \\
750\end{array}$ & $\begin{array}{l}2.156 \\
2.100\end{array}$ & $\begin{array}{r}9 \\
12\end{array}$ & $\begin{array}{l}.2000 \\
.2151 \\
.2267\end{array}$ & $\begin{array}{r}.2004 \\
.2145 \\
.2151 \\
.2267\end{array}$ & $\begin{array}{l}12,0,0 \\
12,2,0 \\
10,1,1 \\
10,3,1\end{array}$ & $\begin{array}{l}1.6897 \\
1.6614 \\
1.6532 \\
1.6292\end{array}$ & $\begin{array}{r}65 \\
5 \\
6 \\
22\end{array}$ & $\begin{array}{l}.3503 \\
.3623 \\
.3659 \\
.3768\end{array}$ & $\begin{array}{l}.3504 \\
.3624 \\
.3658 \\
.3768\end{array}$ & $\begin{array}{r}642 \\
15,5,0 / 13,9,0 \\
14,3,1 / 13,6,1 \\
16,2,0 / 14,8,0\end{array}$ \\
\hline $\begin{array}{l}2.901 \\
2.793 \\
2.769\end{array}$ & $\begin{array}{r}5 \\
82 \\
26\end{array}$ & $\begin{array}{l}.1189 \\
.1282 \\
.1304\end{array}$ & $\begin{array}{l}.1188 \\
.1282 \\
.1304\end{array}$ & $\begin{array}{l}910 \\
541 \\
930\end{array}$ & $\begin{array}{l}2.077 \\
2.015 \\
1.9584\end{array}$ & $\begin{array}{r}5 \\
85 \\
5\end{array}$ & $\begin{array}{l}.2319 \\
.2463 \\
.2607\end{array}$ & $\begin{array}{l}.2319 \\
.2463 \\
.2608\end{array}$ & $\begin{array}{r}12,4,0 \\
13,1,0 / 11,7,0 \\
12,6,0\end{array}$ & $\begin{array}{l}1.5801 \\
1.5688\end{array}$ & $\begin{array}{l}28 \\
48\end{array}$ & $\begin{array}{l}.4005 \\
.4063\end{array}$ & $\begin{array}{l}.4006 \\
.4064\end{array}$ & $\begin{array}{l}15,2,1 \\
13,8,1\end{array}$ \\
\hline
\end{tabular}

${ }^{a}$ Interplaner spacing.

${ }^{b}$ Observed intensity.

${ }^{c}$ Indexed on the basis of a tetragonal unit cell (space group I $\overline{4}$ ) $a=26.270 \AA, c=3.814 \AA$.

Due to the very large monoclinic cell this powder pattern can only be indexed unambiguously with the aid of single crystal data. By utilizing the published $\mathrm{F}_{\text {obs }}$ values for $\mathrm{W}_{5} \mathrm{Nb}_{16} \mathrm{O}_{55}$ [2] it was possible to assign indices to the observed peaks in the powder pattern with reasonable certainty up to about $60^{\circ} 2 \theta$ (Cu radiation). $\quad \mathrm{W}_{5} \mathrm{Nb}_{16} \mathrm{O}_{55}$ is probably not stable below about $1090{ }^{\circ} \mathrm{C}$ and melts incongruently at about $1385{ }^{\circ} \mathrm{C}$.

\section{e. $9 \mathrm{Nb}_{2} \mathrm{O}_{5} \cdot 8 \mathrm{WO}_{3}\left(\mathrm{~W}_{8} \mathrm{Nb}_{18} \mathrm{O}_{69}\right)$}

The unit cell of the last compound observed in this structural series has $n=5, m=5$ [3] and is bodycentered tetragonal with the most probable space group $I \overline{4}$. The indexed $\mathrm{x}$-ray diffraction powder pattern is given in table 5 and the unit cell dimensions derived from this pattern are $a=26.270 \AA, c=3.814 \AA$. This compound is not stable below about $1265{ }^{\circ} \mathrm{C}$ and melts incongruently at about $1375{ }^{\circ} \mathrm{C}$.

It should be noted that the binary equilibrium stability regions of this structural group of compounds tend to decrease with increasing $\mathrm{W}^{+6}$ content. This phenomenon is probably due to the increasing size of the basic "building block" unit, and therefore to the greater energy needed to maintain long range ordering.

\section{f. Other Compounds Structurally Related to $\mathrm{Nb}_{2} \mathrm{O}_{5}$}

In addition to the compounds with structures described by the homologous series formula $\mathrm{B}_{m n p+1}$ $\mathrm{O}_{3 n m p-(n+m) p+4}$, the existence of several other phases has been reported, but their structures are, as yet, unknown. Notable among these is the $\mathrm{NbO}_{2.482}$ (and $\left.(\mathrm{Ti}, \mathrm{Nb}) \mathrm{O}_{2.482}\right)$ reported by Gruehn and Schäfer [22] which is apparently similar to the ' $X$ ' phase reported by Waring and Roth in the system vanadiumoxide-niobium oxide [23]. There are several stable phases in the $\mathrm{Nb}_{2} \mathrm{O}_{5}-\mathrm{WO}_{3}$ system which fall in this category.

\section{g. $13 \mathrm{Nb}_{2} \mathrm{O}_{5} \cdot 4 \mathrm{WO}_{3}\left(\mathrm{~W}_{4} \mathrm{Nb}_{26} \mathrm{O}_{77}\right)$}

Another phase, previously unreported, was found to occur between the known $6: 1$ and $7: 3$ compounds. The composition of this phase was deduced by Schäfer (private communication) to be 13:4 based on oxygen analyses. It was postulated that this compound might have an $\mathrm{Nb}_{2} \mathrm{O}_{5}: \mathrm{WO}_{3}$ ratio of $16: 5$ and the structure would then be related to that of $\mathrm{Nb}_{2} \mathrm{O}_{5}$ by increas ing the size of the basic "building blocks" from $3 \times 4$ and $3 \times 5$ (reported for $\mathrm{Nb}_{2} \mathrm{O}_{5}$ [18]) to $4 \times 4$ and $4 \times 5$. However, single crystals obtained from a 16:5 composition were found by A. D. Wadsley (private communication) to have a unit cell which is $\mathrm{C}$-centered monoclinic $a=29.74 \AA, b=3.823 \AA, c=26.02 \AA$, $\beta=92^{\circ} 18^{\prime}$. These values are not in agreement with the primitive space group and approximate unit cell dimensions which can be derived for the postulated 16:5 structure. The partially indexed powder pattern is given in table 6 . It cannot be fully indexed without the aid of single crystal intensity data due to the very large size of the unit cell. The structure of this phase, is actually a mixture of blocks of $3 \times 4$ and $4 \times 4$ occurring in alternate sequence to make an "ordered intergrowth" structure [24]. The composition can be arrived at by adding the homologous series twice (as for $\mathrm{Nb}_{2} \mathrm{O}_{5}$ [4]) $n=3, m=4, p=1\left(\mathrm{~B}_{13} \mathrm{O}_{33}\right)$ plus $n=4, m=4, p=1\left(\mathrm{~B}_{17} \mathrm{O}_{44}\right)=\mathrm{B}_{30} \mathrm{O}_{77}\left(13 \mathrm{Nb}_{2} \mathrm{O}_{5} \cdot 4 \mathrm{WO}_{3}\right)$. Although this compound does not form in short-time experiments (about $\mathrm{l} \mathrm{hr}$ ) it can be prepared readily by heating for several days at about $1400{ }^{\circ} \mathrm{C}$. This phase was observed to decompose at about $1435{ }^{\circ} \mathrm{C}$ into $6 \mathrm{Nb}_{2} \mathrm{O}_{5} \cdot \mathrm{WO}_{3}$ and $7 \mathrm{Nb}_{2} \mathrm{O}_{5} \cdot 3 \mathrm{WO}_{3}$.

\section{h. "3ONb $\mathrm{O}_{5}: \mathrm{WO}_{3}{ }^{2}$}

Another phase was found to occur in the $\mathrm{Nb}_{2} \mathrm{O}_{5}$ $\mathrm{WO}_{3}$ system at a ratio of approximately 3.5 mole

${ }^{2}$ Quotation marks are used around a composition whenever the phase referred to has not been completely characterized. 
TABLE 6. X-ray diffraction powder data for the compound $\mathrm{W}_{4} \mathrm{Nb}_{26} \mathrm{O}_{77}$ $\left(\right.$ CuK $K_{\alpha}$ radiation $)$

\begin{tabular}{|c|c|c|c|c|c|}
\hline$d^{\mathrm{a}}$ & $I^{\mathrm{b}}$ & $h k l^{c}$ & $d^{\mathrm{a}}$ & $I^{\mathrm{b}}$ & $h k l^{c}$ \\
\hline 14.82 & 10 & 200 & 2.418 & 10 & \\
\hline 12.93 & 8 & 002 & 2.346 & 7 & \\
\hline 7.43 & 12 & 400 & 2.309 & 22 & \\
\hline 6.55 & 5 & & 2.277 & 5 & \\
\hline 5.19 & 7 & & 2.113 & 7 & \\
\hline 4.951 & 14 & 600 & 2.067 & 13 & \\
\hline 4.833 & 15 & 205 & 2.055 & 42 & \\
\hline 4.686 & 60 & $60 \overline{2}$ & 2.042 & 88 & \\
\hline 3.786 & 18 & 110 & 1.912 & 68 & 020 \\
\hline 3.748 & 200 & $\left\{\begin{array}{l}11 \overline{1} \\
111\end{array}\right.$ & 1.857 & 12 & \\
\hline & & $\int 007$ & $\begin{array}{l}1.854 \\
1.848\end{array}$ & $\begin{array}{l}15 \\
25\end{array}$ & \\
\hline 3.711 & 40 & $\{800$ & $\begin{array}{l}1.040 \\
1.835\end{array}$ & 5 & \\
\hline 3.657 & 15 & $\left\{\begin{array}{l}112 \\
207\end{array}\right.$ & 1.816 & 5 & \\
\hline 3.610 & 250 & $80 \frac{1}{20}$ & 1.805 & 33 & \\
\hline 3.564 & 190 & 207 & 1.781 & 16 & \\
\hline 3.542 & 50 & $\left\{\begin{array}{l}802 \\
313\end{array}\right.$ & 1.763 & 15 & \\
\hline & & [311 & 1.756 & 19 & $i$ \\
\hline $\begin{array}{l}3.463 \\
3.367\end{array}$ & 10 & 113 & $\begin{array}{l}1.744 \\
1.736\end{array}$ & $\begin{array}{l}13 \\
16\end{array}$ & \\
\hline $\begin{array}{l}3.367 \\
3.264\end{array}$ & $\begin{array}{l}10 \\
10\end{array}$ & & & & \\
\hline $\begin{array}{l}3.264 \\
3.199\end{array}$ & $\begin{array}{l}10 \\
16\end{array}$ & 114 & 1.731 & 15 & \\
\hline 2.966 & 5 & & $\begin{array}{l}1.706 \\
1689\end{array}$ & $\begin{array}{r}5 \\
25\end{array}$ & \\
\hline 2.919 & 15 & & $\begin{array}{l}1.009 \\
1.684\end{array}$ & 68 & \\
\hline 2.845 & 32 & & 1.675 & 12 & \\
\hline 2.832 & 48 & & & & \\
\hline 2.777 & 10 & & 1.610 & 25 & \\
\hline 2.731 & 52 & & $\begin{array}{l}1.597 \\
1.592\end{array}$ & $\begin{array}{r}8 \\
20\end{array}$ & \\
\hline \multirow{3}{*}{$\begin{array}{l}2.724 \\
2.529\end{array}$} & 55 & & 1.585 & 18 & \\
\hline & 90 & & & 25 & \\
\hline & & & $\mid \begin{array}{l}1.5 / 1 \\
1.565\end{array}$ & 38 & \\
\hline
\end{tabular}

a Interplaner spacing.

b Observed in

c Partially indexed on the basis of a C-centered monoclinic unit cell with $a=29.74 \AA$ $b=3.823 \AA, c=26.02 \AA, \beta=92^{\circ} 18^{\prime}$ by comparison with the single crystal intensities observed from a film taken with a Weisingberg camera [A. D. Wadsley, private communication].

percent $\mathrm{WO}_{3}$. This phase is apparently the same as that previously reported to occur at about $15: 1$, the structure of which could not be verified [1]. The $30: 1$ ratio is that deduced by Schäfer (private communication). It is apparently composed of some complex packing of the "building blocks" previously described. One possible structure might be $n=3$, $m=4, p=2\left(\mathrm{~B}_{25} \mathrm{O}_{62}\right)$ plus two blocks of $n=3, m=4$, $p=1 \quad\left(\mathrm{~B}_{25} \mathrm{O}_{66}\right)=\mathrm{B}_{51} \mathrm{O}_{128} \quad\left(25 \mathrm{Nb}_{2} \mathrm{O}_{5} \cdot \mathrm{WO}_{3}\right)$. Another possibility might be $n=3, m=4, p=2\left(\mathrm{~B}_{25} \mathrm{O}_{62}\right)+n=3$, $m=5, \quad p=1 \quad\left(\mathrm{~B}_{16} \mathrm{O}_{41}\right)=\mathrm{B}_{41} \mathrm{O}_{105} \quad\left(20 \mathrm{Nb}_{2} \mathrm{O}_{5} \cdot \mathrm{WO}_{3}\right)$. The exact structure and composition remain to be proven because no good single crystal data is yet available. The "30:1" phase was found to melt incongruently at about $1470{ }^{\circ} \mathrm{C}$. Its unindexed x-ray diffraction powder pattern is listed in table 7 .

\subsection{Compounds Containing Pentagonal Bipyramid (Sevenfold) Coordinated Cations}

\section{a. $\mathrm{Nb}_{2} \mathrm{O}_{5} \cdot \mathrm{WO}_{3}\left(\mathrm{WNb}_{2} \mathrm{O}_{8}\right)$}

A phase isostructural with $\mathrm{Nb}_{3} \mathrm{O}_{7} \mathrm{~F}$ [25] might be thought likely to occur in this system, but no such structure has ever been reported in pure oxide systems.
TABLE 7. X-ray diffraction powder data for the composition $30 \mathrm{Nb}_{2} \mathrm{O}_{5}: \mathrm{WO}_{3}\left(\right.$ CuK $K_{\alpha}$ radiation $)$

\begin{tabular}{|c|c|c|c|}
\hline$d^{a}$ & $I^{b}$ & $d^{a}$ & $I^{b}$ \\
\hline 16.20 & 10 & 2.435 & 9 \\
\hline 13.93 & 4 & 2.376 & 40 \\
\hline 10.73 & 16 & 2.212 & 6 \\
\hline 8.96 & 7 & 2.100 & 8 \\
\hline 8.10 & 5 & 2.076 & 74 \\
\hline 6.37 & 14 & 2.041 & 90 \\
\hline 5.36 & 8 & 1.999 & 5 \\
\hline 5.10 & 50 & 1.936 & 8 \\
\hline 4.797 & 7 & 1.913 & 48 \\
\hline 4.645 & 60 & 1.871 & 10 \\
\hline 3.742 & 132 & 1.866 & 10 \\
\hline 3.638 & 312 & 1.857 & 12 \\
\hline 3.579 & 10 & 1.825 & 18 \\
\hline 3.556 & 10 & 1.819 & 42 \\
\hline 3.493 & 228 & 1.796 & 8 \\
\hline 3.354 & 37 & 1.791 & 8 \\
\hline 3.173 & 16 & 1.782 & 23 \\
\hline 3.093 & 7 & 1.769 & 7 \\
\hline 2.984 & 14 & 1.746 & 14 \\
\hline 2.845 & 55 & 1.743 & 25 \\
\hline 2.836 & 47 & 1.731 & 30 \\
\hline 2.763 & 38 & 1.716 & 10 \\
\hline 2.707 & 46 & 1.693 & 22 \\
\hline 2.693 & 18 & 1.684 & 30 \\
\hline 2.647 & 8 & 1.680 & 60 \\
\hline 2.614 & 7 & 1.623 & 15 \\
\hline 2.597 & 6 & 1.603 & 8 \\
\hline 2.538 & 65 & 1.595 & 30 \\
\hline 2.503 & 45 & 1.581 & 44 \\
\hline 2.476 & 10 & 1.558 & 28 \\
\hline 2.458 & 10 & 1.548 & 8 \\
\hline
\end{tabular}

Instead a new phase was found with unit cell dimensions related to the tetragonal tungsten bronze-type structures. Single crystals of the 1:1 compound were prepared by decomposing the 9:8 phase (made with the less pure end members) at about $1100^{\circ} \mathrm{C}$. Single crystal precession patterns of this phase made by $\mathrm{A}$. Perloff of the National Bureau of Standards staff showed these crystals to be orthorhombic, probable space groups Pmab or $\mathrm{P} 2_{1} \mathrm{ab}$. From these data the $\mathrm{x}$-ray diffraction powder pattern given in table 8 was indexed with $a=16.615 \AA, b=17.616 \AA, c=3.955 \AA$. The compound $\mathrm{Nb}_{2} \mathrm{O}_{5} \cdot \mathrm{WO}_{3}$ could not be made as a single phase in the quenching experiments. X-ray diffraction powder patterns of these specimens always showed a trace of a metastable bronze-type phase plus some $7 \mathrm{Nb}_{2} \mathrm{O}_{5} \cdot 3 \mathrm{WO}_{3}$. However, high temperature $\mathrm{x}$-ray powder patterns showed that the extra phases disappeared quickly above about $900{ }^{\circ} \mathrm{C}$. The $1: 1$ compound was found to dissociate at about $1115^{\circ} \mathrm{C}$ to $8 \mathrm{Nb}_{2} \mathrm{O}_{5} \cdot 5 \mathrm{WO}_{3}$ plus a bronze-like phase, probably $4 \mathrm{Nb}_{2} \mathrm{O}_{5} \cdot 9 \mathrm{WO}_{3}$.

$\mathrm{WNb}_{2} \mathrm{O}_{8}$ is related to $\mathrm{WTa}_{2} \mathrm{O}_{8} \quad(a=16.701 \AA$, $b=8.864 \AA, c=3.877 \AA$ ) by a doubled $b$-axis. The crystal structure of $\mathrm{LiNb}_{6} \mathrm{O}_{15} \mathrm{~F}$ which is apparently isostructural with $\mathrm{WTa}_{2} \mathrm{O}_{8}$ was found by $\mathrm{S}$. Andersson and M. Lundberg [26] to be made up of octahedrally coordinated cations. These octahedra are corner shared to form a ring of five octahedra. Within this ring is a cation in sevenfold coordination, in a penta- 
TABLE 8. X-ray diffraction powder data for the compound $\mathrm{WNb}_{2} \mathrm{O}_{8}\left(C u K_{\alpha}\right.$ radiation)

\begin{tabular}{|c|c|c|c|c|c|c|c|c|c|c|c|c|c|c|}
\hline$d^{\mathrm{a}}$ & $I^{\mathrm{b}}$ & $\frac{1}{d^{2}}$ obs & $\frac{1}{d^{2}}$ calc & $h k l^{c}$ & $d^{\mathrm{a}}$ & $I^{\mathrm{b}}$ & $\frac{1}{d^{2}}$ obs & $\frac{1}{d^{2}}$ calc & $h k l^{c}$ & $d^{n}$ & $I^{\mathrm{b}}$ & $\frac{1}{d^{2}}$ obs & $\frac{1}{d^{2}}$ calc & $h k l^{\mathrm{c}}$ \\
\hline 8.83 & 6 & 0.0128 & 0.0129 & 020 & 2.250 & 8 & .1976 & .1975 & 611 & 1.7546 & 16 & .3248 & $\left\{\begin{array}{l}3246 \\
3240\end{array}\right.$ & 831 \\
\hline 8.30 & 25 & .0145 & .0145 & 200 & 2.223 & 8 & .2023 & .2024 & 451 & 1.7496 & 20 & .3267 & $\begin{array}{r}.3249 \\
3266\end{array}$ & $\begin{array}{l}091 \\
422\end{array}$ \\
\hline 4.689 & 6 & .0455 & .0455 & 320 & 2.203 & 19 & .2061 & $\left\{\begin{array}{r}.2060 \\
.066\end{array}\right.$ & 541 & $\begin{array}{l}1.7490 \\
1.7464\end{array}$ & $\begin{array}{l}20 \\
23\end{array}$ & $\begin{array}{l}.3207 \\
.3279\end{array}$ & .3281 & 481 \\
\hline 4.403 & 5 & .0516 & .0516 & 040 & 2.200 & 13 & .2001 & |. 2062 & 080 & 10. & 15 & 3362 & $\int .3363$ & 052 \\
\hline 4.259 & 12 & .0551 & .0552 & 140 & $\begin{array}{l}2.198 \\
2.117\end{array}$ & $\begin{array}{r}19 \\
4\end{array}$ & $\begin{array}{l}.2070 \\
.2232\end{array}$ & $\begin{array}{l}.2072 \\
.2233\end{array}$ & $\begin{array}{l}621 \\
631\end{array}$ & 1.7247 & 15 & .3362 & $\begin{array}{l}.3366 \\
.3394\end{array}$ & $\begin{array}{l}680 \\
291\end{array}$ \\
\hline 3.952 & 272 & .0640 & .0639 & 001 & 2.091 & 8 & .2288 & .2290 & 740 & 1.7154 & 16 & .3398 & $\left\{\begin{array}{l}.3399 \\
.3399\end{array}\right.$ & 342 \\
\hline 3.894 & 46 & .0660 & .0660 & 240 & & & & & & 10.107 & 10 & & .3399 & 152 \\
\hline 3.759 & 112 & .0708 & .0708 & 420 & 2.078 & 14 & .2316 & $\begin{array}{r}.2317 \\
2370\end{array}$ & 800 & & & & & \\
\hline 3.608 & 4 & .0768 & .0768 & 021 & 2.050 & 6 & .2381 & .2379 & 461 & 16969 & & & $\{.3472$ & 841 \\
\hline 3.570 & 9 & .0785 & .0785 & 201 & 2.023 & 18 & .2443 & $\left\{\begin{array}{l}.2445 \\
.2446\end{array}\right.$ & $\begin{array}{l}711 \\
820\end{array}$ & 1.6969 & 40 & $\begin{array}{r}.3473 \\
3507\end{array}$ & $\begin{array}{r}.3477 \\
3508\end{array}$ & $\begin{array}{l}860 \\
252\end{array}$ \\
\hline 3.449 & 202 & .0841 & .0841 & 340 & 1.9774 & 50 & .2558 & .2557 & 002 & $\begin{array}{l}1.0005 \\
1.6740\end{array}$ & 7 & .3569 & $\begin{array}{l}.0300 \\
.3573\end{array}$ & 761 \\
\hline 3.279 & 20 & .0930 & .0929 & 031 & 1.9652 & 7 & .2589 & .2590 & 012 & 1.6684 & 22 & .3593 & .3591 & 522 \\
\hline 3.215 & 8 & .0967 & .0966 & 131 & & & & & & 1.6662 & 28 & .3602 & .3604 & 911 \\
\hline 3.165 & 22 & .0998 & $\begin{array}{l}.0997 \\
1034\end{array}$ & 311 & 1.9473 & 18 & .2637 & $\begin{array}{r}.2642 \\
2702\end{array}$ & $\begin{array}{l}480 \\
081\end{array}$ & & & & & \\
\hline 3.110 & 98 & .1034 & .1034 & & 1.9247 & 13 & .2699 & $\left\{\begin{array}{l}.2702 \\
.2702\end{array}\right.$ & $\begin{array}{l}001 \\
202\end{array}$ & $\begin{array}{l}1.6651 \\
1.6625\end{array}$ & $\begin{array}{l}27 \\
26\end{array}$ & $\begin{array}{l}.3607 \\
.3618\end{array}$ & $\begin{array}{l}.3607 \\
.3620\end{array}$ & $\begin{array}{r}581 \\
10,0,0\end{array}$ \\
\hline 3.051 & 16 & .1075 & .1074 & 231 & & & & .2703 & 731 & 1.6548 & 5 & .3652 & .3652 & $\begin{array}{r}442 \\
\end{array}$ \\
\hline 2.937 & 4 & .1159 & .1160 & 060 & 1.8797 & 16 & .2830 & $\{.2831$ & 222 & 1.6434 & 4 & .3703 & .3701 & 921 \\
\hline 2.893 & 80 & .1195 & .1196 & 160 & & & & .2833 & 840 & 1.6396 & 4 & .3720 & 3717 & 062 \\
\hline 2.825 & 11 & .1253 & $\left\{\begin{array}{l}.1251 \\
.1255\end{array}\right.$ & $\begin{array}{l}411 \\
331\end{array}$ & 1.8743 & 15 & .2847 & $\left\{\begin{array}{r}.2846 \\
2847\end{array}\right.$ & $\begin{array}{l}281 \\
032\end{array}$ & 1 6326 & 12 & 2752 & $\mid \begin{array}{l}.3749 \\
375 ?\end{array}$ & $10,2,0$ \\
\hline 2.771 & 78 & 1303 & .1303 & 600 & 1.8521 & 18 & .2915 & .2915 & 312 & 1.0320 & 12 & .3152 & $\left\{\begin{array}{l}.0702 \\
.3754\end{array}\right.$ & $\begin{array}{l}502 \\
162\end{array}$ \\
\hline 2.725 & 50 & 1347 & $\left\{\begin{array}{l}.1305 \\
1348\end{array}\right.$ & 260 & 8475 & 24 & 2030 & $\int .2929$ & 741 & & & & & \\
\hline 2.654 & 16 & .1420 & .1421 & 540 & 1.078 & 29 & .2900 & .2934 & 760 & 1.6234 & 14 & .3795 & $\begin{array}{r}.5802 \\
.3861\end{array}$ & $\begin{array}{r}4,10,0 \\
602\end{array}$ \\
\hline 2.644 & 18 & .1430 & .1432 & 620 & 1.8373 & 30 & .2962 & $\left\{\begin{array}{r}.2956 \\
2968\end{array}\right.$ & $\begin{array}{l}801 \\
580\end{array}$ & & & & $\{.3861$ & $0.10,1$ \\
\hline 2.633 & 12 & .1443 & .1445 & 051 & & & & $\begin{array}{l}.2908 \\
.2988\end{array}$ & $\begin{array}{l}580 \\
811\end{array}$ & 1.6096 & 13 & .3860 & .3862 & 931 \\
\hline 2599 & & & $\int .1481$ & 341 & 1.8284 & 15 & .2991 & $\{.2992$ & 232 & & & & $\begin{array}{r}.3862 \\
.3893\end{array}$ & $\begin{array}{l}262 \\
612\end{array}$ \\
\hline 2.599 & 86 & .1481 & $\{.1481$ & 151 & $\begin{array}{l}1.8178 \\
1.8081\end{array}$ & 6 & $\begin{array}{r}.3026 \\
3059\end{array}$ & $\begin{array}{r}.3027 \\
3061\end{array}$ & $\begin{array}{l}381 \\
920\end{array}$ & 1.6029 & 9 & .3892 & $\left\{\begin{array}{l}.3898 \\
\text { S }\end{array}\right.$ & $1,10,1$ \\
\hline 2.573 & 8 & .1511 & .1509 & 431 & 1.8081 & & & .3001 & 920 & 1.5928 & 6 & .3942 & .3942 & 452 \\
\hline 2.518 & 8 & .1577 & .1577 & 511 & & & & $\int .3073$ & 042 & 1.5853 & 8 & .3979 & .3978 & 542 \\
\hline $\begin{array}{l}2.510 \\
2.445\end{array}$ & 8 & .1587 & $\begin{array}{r}.1590 \\
1673\end{array}$ & 251 & 1.8017 & 8 & .3081 & $\{.3085$ & 821 & & & & & \\
\hline & 44 & $.16 / 3$ & $.10 / 3$ & & 1.7908 & 7 & .3118 & .3109 & 142 & 1.5826 & 8 & .3993 & $\left\{\begin{array}{l}3990 \\
3992\end{array}\right.$ & $\begin{array}{l}622 \\
771\end{array}$ \\
\hline 2.398 & 7 & .1739 & .1739 & 460 & 1.7852 & 6 & .3138 & $\begin{array}{r}.3123 \\
.3137\end{array}$ & $\begin{array}{l}571 \\
402\end{array}$ & 15808 & 10 & 4002 & .4005 & 681 \\
\hline 2.375 & 8 & .1773 & .1771 & 351 & $\begin{array}{l}1.7852 \\
1.7762\end{array}$ & 9 & $\begin{array}{l}.0130 \\
.3170\end{array}$ & .3169 & 412 & 1.5808 & 10 & $.400 \mathrm{Z}$ & $\{.4006$ & $2,10,1$ \\
\hline 2.334 & 40 & .1835 & $\left\{\begin{array}{l}.1834 \\
.1836\end{array}\right.$ & $\begin{array}{l}531 \\
161\end{array}$ & & & & .3218 & 242 & 1.5640 & 14 & .4088 & $\left\{\begin{array}{r}.4087 \\
4093\end{array}\right.$ & 941 \\
\hline 2.293 & 5 & .1901 & .1903 & 720 & 1.7637 & 14 & .3215 & $\left\{\begin{array}{r}3219 \\
3223\end{array}\right.$ & $\begin{array}{r}751 \\
0.10 .0\end{array}$ & 1.5592 & 26 & .4114 & .4116 & 861 \\
\hline 2.268 & 23 & .1943 & $\left\{\begin{array}{l}.1943 \\
.1944\end{array}\right.$ & $\begin{array}{l}601 \\
261\end{array}$ & & & & & & 1.5326 & 12 & .4257 & .4260 & $10,0,1$ \\
\hline
\end{tabular}

gonal bipyramid configuration. These rings are connected by further corner sharing to form double chains. The doubled cell in $\mathrm{WNb}_{2} \mathrm{O}_{8}$ is probably due to an alternate puckering of the positions occupied by the sevenfold coordinated ions, but the proof of this must await a complete single crystal structure analysis.

\section{b. $4 \mathrm{Nb}_{2} \mathrm{O}_{5} \cdot 9 \mathrm{WO}_{3}\left(\mathrm{Nb}_{8} \mathrm{~W}_{9} \mathrm{O}_{47}\right)$}

A compound having a general diffraction pattern similar to tetragonal potassium tungsten bronze $\left(\mathrm{K}_{x} \mathrm{WO}_{3}\right)$ was reported by Roth and Wadsley [1] to occur at the composition $4 \mathrm{Nb}_{2} \mathrm{O}_{5} \cdot 9 \mathrm{WO}_{3}$. Sleight and Magneli [27] described the structure of this phase from a crystal obtained from a $1: 3$ composition which had been heated to a temperature of $1200-1400{ }^{\circ} \mathrm{C}$. The structure of this crystal was found to be made up of three tetragonal-bronze-like unit cells with four out of the twelve possible five-fold rings being occupied by cations, with oxygens above and below forming pentagonal bipyramid coordination polyhedra. This structure represents the composition $\mathrm{Nb}_{16} \mathrm{~W}_{18} \mathrm{O}_{94}$ and has orthorhombic symmetry. The unit cell dimensions found in the present work are $a=36.692 \AA$, $b=12.191 \AA, c=3.945 \AA$, and were obtained from the indexed x-ray diffraction powder pattern given in table 9. This compound was found to be stable from at least $1150{ }^{\circ} \mathrm{C}$ to the congruent melting point of about $1380{ }^{\circ} \mathrm{C}$.

The presence of cations in the pentagonal holes of the tetragonal tungsten bronze structure suggests a possible homologous series which can be expressed as:

$$
n \mathrm{~B}_{10} \mathrm{O}_{30}+m \mathrm{BO} \text { or } \mathrm{B}_{10 n+m} \mathrm{O}_{30 n+m}
$$

where $n$ is the number of subcells of the tetragonal bronze type in the true unit cell and $m$ is the number of pentagonal bipyramid polyhedra occupied by a cation. Each bronze-type subcell has only four pentagonal holes, therefore the maximum value of $m$ must be equal to or less than $4 n$.

\section{c. $2 \mathrm{Nb}_{2} \mathrm{O}_{5} \cdot \mathbf{T} \mathrm{WO}_{3}\left(\mathrm{Nb}_{4} \mathbf{W}_{7} \mathrm{O}_{31}\right)$}

One method of reducing the general formula $\mathrm{B}_{10 n+m} \mathrm{O}_{30 n+m}$ to include the $4: 9$ compound and to predict a minimum number of other phases is to assume $m=4$. This results in the formula $\mathrm{B}_{10 n+4} \mathrm{O}_{30 n+4}$. 
TABLE 9. X-ray diffraction powder data for the compound $\mathrm{W}_{9} \mathrm{Nb}_{8} \mathrm{O}_{47}$ (CuK $\mathrm{K}_{\alpha}$ radiation)

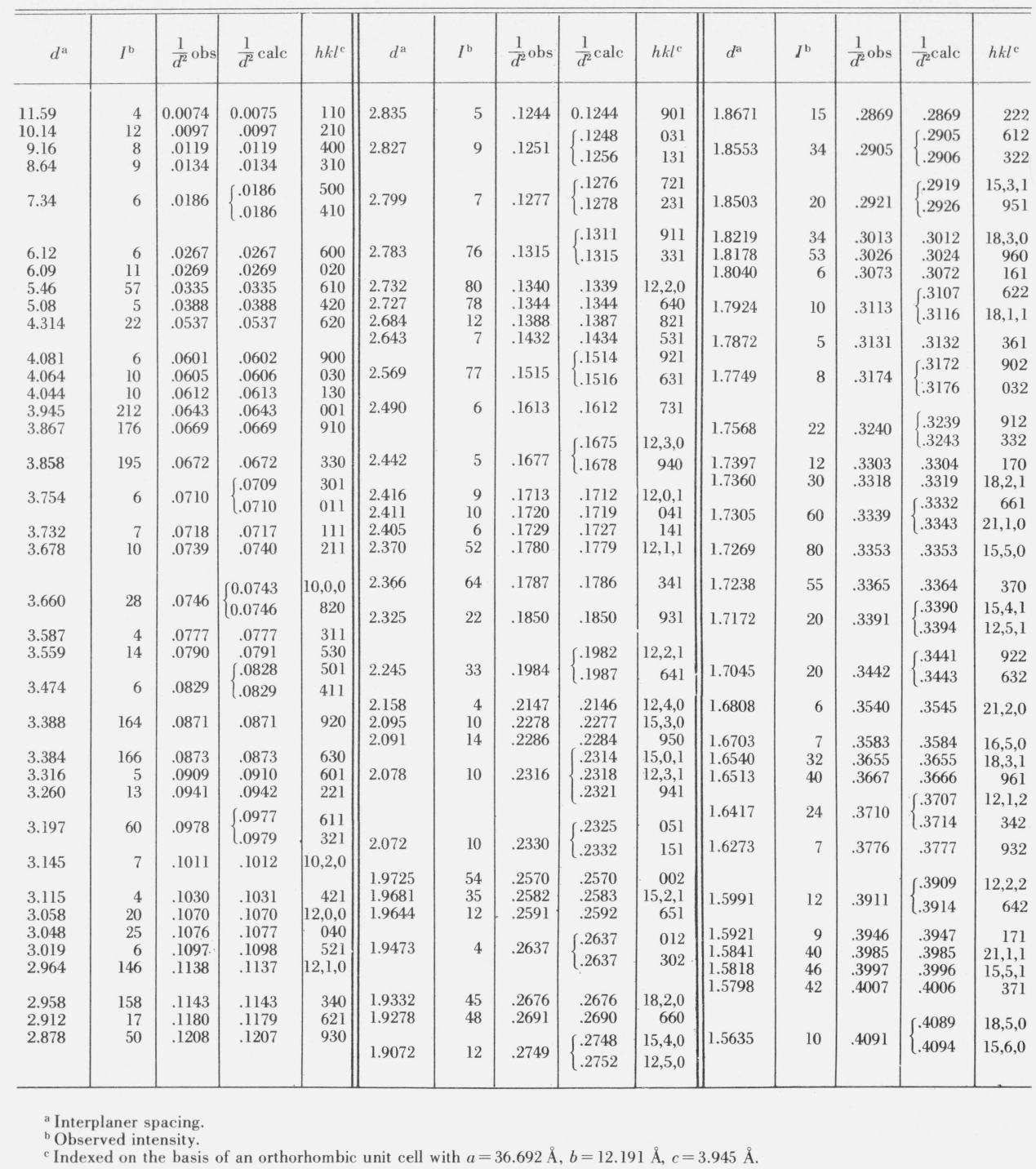

For the $4: 9$ compound $n=3$. The composition of $n=2$ is $\mathrm{Nb}_{2} \mathrm{O}_{5} \cdot \mathrm{WO}_{3}$ and the composition of $n=4$ would be $2 \mathrm{Nb}_{2} \mathrm{O}_{5}: 7 \mathrm{WO}_{3}$. The $1: 1$ compound does not belong to the general homologous series but has a unique structure only vaguely related to the tetragonal tungsten bronze type, as previously described. The structure predicted for $2 \mathrm{Nb}_{2} \mathrm{O}_{5} \cdot 7 \mathrm{WO}_{3}$ would be either orthorhombic with one $a$-axis multiplied by four or tetragonal with both $a$-axes doubled. The latter structure, which exhibits a higher symmetry than the former, was found to occur for the $2: 7 \mathrm{com}$ position between a minimum temperature of about $1245{ }^{\circ} \mathrm{C}$ and the probable congruent melting point of $1357{ }^{\circ} \mathrm{C}$. A crystal structure analysis of this compound is currently being conducted by N. Stephensen [28]. The unit cell dimensions of $\mathrm{Nb}_{4} \mathrm{~W}_{7} \mathrm{O}_{31}$ are $a=24.264 \AA, c=3.924 \AA$, and were obtained from the indexed x-ray diffraction powder pattern listed in table 10.

\section{d. "6 $6 \mathrm{Nb}_{2} \mathrm{O}_{5} \cdot 11 \mathrm{WO}_{3}$ " $\left(\mathrm{Nb}_{12} \mathrm{~W}_{11} \mathrm{O}_{63}\right)$}

Roth and Wadsley [1] reported another compound in this system occurring at a ratio of about $13 \mathrm{Nb}_{2} \mathrm{O}_{5}: 24 \mathrm{WO}_{3}$. Kovba and Trunov [9] described a phase to which they assigned the composition $4 \mathrm{Nb}_{2} \mathrm{O}_{5}: 7 \mathrm{WO}_{3}$. The only logical ratio, near this composition, which could belong to the general formula $\mathrm{B}_{10 n+m} \mathrm{O}_{30 n+m}$ is $6 \mathrm{Nb}_{2} \mathrm{O}_{5}: 11 \mathrm{WO}_{3}$. In order to include this composition with the $4: 9$ and $2: 7$ compounds in one simplified formula, it is sufficient to postulate a series limited to the case $m=n+1$. The new homologous series formula then becomes $\mathrm{B}_{11 n+1} \mathrm{O}_{31 n+1}$. In this case for the $2: 7$ compound, $n=\infty$ and for $4 \mathrm{Nb}_{2} \mathrm{O}_{5} \cdot 9 \mathrm{WO}_{3}, n=3$. When $n=2$ the composition is $\mathrm{B}_{23} \mathrm{O}_{63}$ and the predicted structure has two bronze-like subcells in the unit cell, with three filled pentagonal holes. Such a structure would have a very low symmetry and is apparently not formed 
Table 10. X-ray diffraction powder data for the compound $\mathrm{W}_{7} \mathrm{Nb}_{4} \mathrm{O}_{31}\left(\right.$ CuK $K_{\alpha}$ radiation $)$

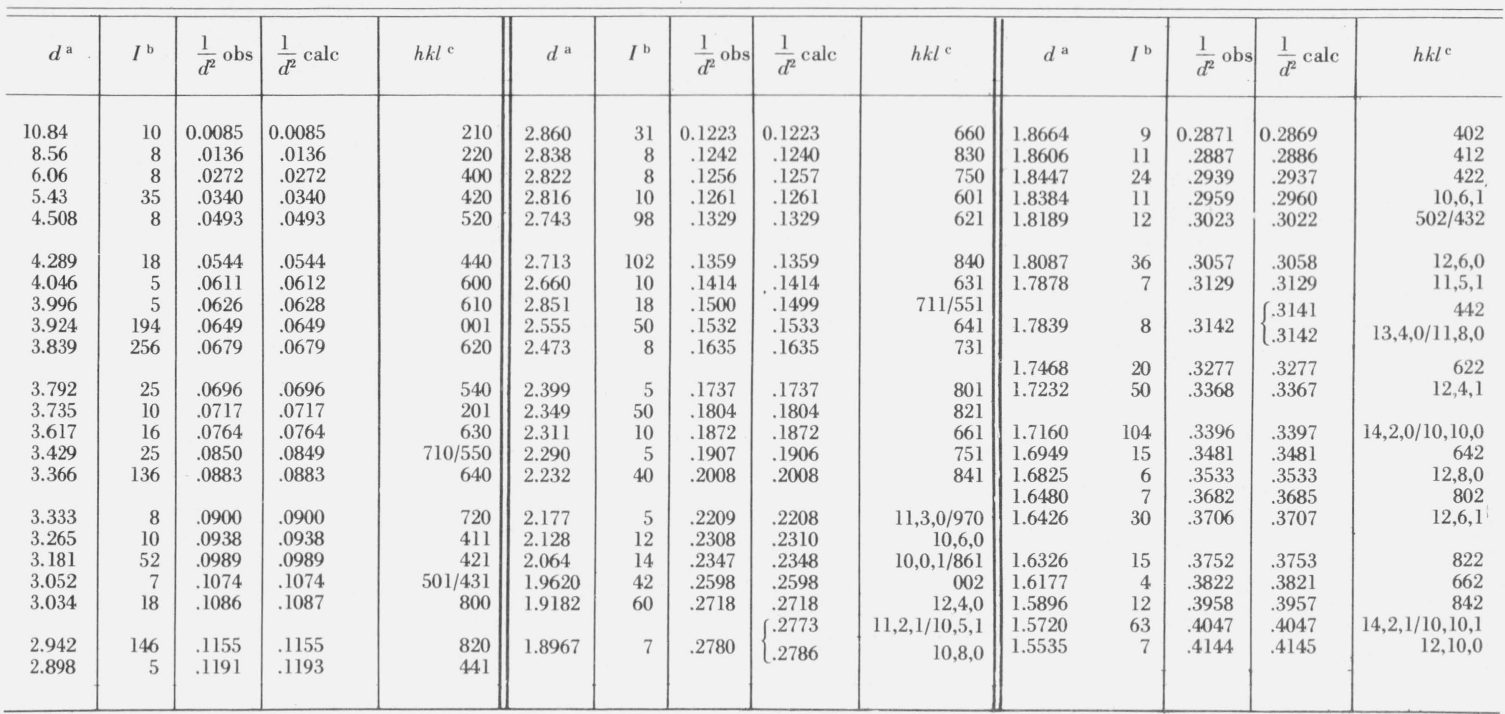

a Interplanar spacing

b Observed intensity.

c Indexed on the basis of a tetragonal unit cell with $a=24.264 \AA, c=3.924 \AA$.

in this system. Experimentally the compound " $6 \mathrm{Nb}_{2} \mathrm{O}_{5} \cdot 11 \mathrm{WO}_{3}$ " was found to be orthorhombic, probably with the $a$-axis of the tetragonal bronze subcell tripled. It is stable from about $1210{ }^{\circ} \mathrm{C}$ to the apparently congruent melting point of $1378{ }^{\circ} \mathrm{C}$. The x-ray diffraction powder pattern listed in table 11 is indexed with $a=36.740 \AA, b=12.195 \AA, c=3.951 \AA$. It may be concluded from the size and symmetry of the unit cell, that the homologous series formula $\mathrm{B}_{11 n+1} \mathrm{O}_{31 n+1}$ is not correct for this compound. Therefore, " $6 \mathrm{Nb}_{2} \mathrm{O}_{5} \cdot 11 \mathrm{WO}_{3}$ " either has a defect structure or belongs to some other structural homologous series, as yet unknown. A single crystal structure analysis is currently under study by N. Stephenson [28].

\section{e. Metastable Tetragonal Bronze-Type Solid Solution ("3:8")}

In addition to the ordered bronze-like phases which have been found in the $\mathrm{Nb}_{2} \mathrm{O}_{5}-\mathrm{WO}_{3}$ system, another, apparently disordered, phase has also been reported at about the $\mathrm{Nb}_{2} \mathrm{O}_{5}: 3 \mathrm{WO}_{3}$ composition [5, 6, 7]. In the present study a tetragonal bronze-type phase without any indication of superstructure in the powder pattern was found to occur from about 72 to 74 mole percent $\mathrm{WO}_{3}$ in a temperature range from about 1100 to $1250{ }^{\circ} \mathrm{C}$. The unit cell dimensions of this phase were found to vary from about $a=12.190 \AA$, $c=3.968 \AA$ for the composition containing 72 mole percent $\mathrm{WO}_{3}$ to $a=12.178 \AA, c=3.930 \AA$ for the composition containing 75 mole percent $\mathrm{WO}_{3}$. It must be concluded that the lower temperature phase is only metastable, because it decomposes at high temperatures into the two ordered compounds, $4 \mathrm{Nb}_{2} \mathrm{O}_{5} \cdot 9 \mathrm{WO}_{3}$ and $2 \mathrm{Nb}_{2} \mathrm{O}_{5} \cdot 7 \mathrm{WO}_{3}$.

\subsection{Compounds Related to the $\mathrm{ReO}_{3}$ Structure-Type. The Magneli "Shear Phases"}

In the temperature interval from about 1270 to $1358{ }^{\circ} \mathrm{C}$, at least two phases have been found to occur with $\mathrm{x}$-ray diffraction powder patterns suggestive of the Magneli "shear phases" [29], which are structurally related to $\mathrm{WO}_{3}$. These phases have been found to occur between about 91 and 94 mole percent $\mathrm{WO}_{3}$. Due to the difficulty of obtaining equilibrium and the complex nature of the diffraction patterns, it is very difficult to decipher the exact composition of the phases by powder data alone. Although no single crystals have been examined, the best interpretation of the data suggests that there are two equilibrium phases having the $\mathrm{Nb}_{2} \mathrm{O}_{5}: \mathrm{WO}_{3}$ ratios of 1:11 and 1:15. These compositions would correspond to the members $n=13$ and $n=17$ of the homologous series $\mathrm{B}_{n} \mathrm{O}_{3 n-1}$. It is possible that other structurally related phases are formed in this compositional region which have very little or no thermal stability. Unindexed x-ray diffraction powder patterns for the " $1: 11$ " and " $1: 15$ " phases are given in tables 12 and 13 , respectively.

\subsection{Polymorphs of $\mathbf{W O}_{3}$ and $\mathbf{W O}_{3}$ Solid Solutions}

$\mathrm{WO}_{3}$ has been reported to occur in many different polymorphs [30-32]. None of the various high- or low-temperature polymorphs have been previously reported to be quenchable to room temperature. However, many of these reported phases have been found at room temperature in the $\mathrm{Nb}_{2} \mathrm{O}_{5}-\mathrm{WO}_{3}$ system. 
TABLE 11. X-ray diffraction powder data for the phase $" 6 \mathrm{Nb}_{2} \mathrm{O}_{5} \cdot 11 \mathrm{WO}_{3} "\left(\right.$ CuK $K_{a}$ radiation $)$

\begin{tabular}{|c|c|c|c|c|c|c|c|c|c|}
\hline$d^{\mathrm{a}}$ & $I^{\mathrm{b}}$ & $\frac{1}{d^{2}}$ obs & $\frac{1}{d^{2}}$ calc & $h k l^{\mathrm{c}}$ & $d^{\text {a }}$ & $I^{\mathrm{b}}$ & $\frac{1}{d^{2}}$ obs & $\frac{1}{d^{2}}$ calc & $h k l^{\mathrm{c}}$ \\
\hline 11.62 & 5 & 0.0074 & 0.0075 & 110 & 2.328 & 15 & .1846 & .1846 & 931 \\
\hline 10.18 & 14 & .0097 & .0097 & 210 & 2.248 & 25 & 1978 & .1977 & $12,2,1$ \\
\hline 9.16 & 8 & .0119 & .0119 & 400 & 2.246 & 22 & .1983 & .1983 & 641 \\
\hline 8.62 & 8 & .0135 & .0134 & 310 & 2.097 & 7 & .2273 & .2272 & $15,3,0$ \\
\hline 5.47 & 36 & .0334 & .0334 & 610 & 2.095 & 8 & .2282 & .2281 & 950 \\
\hline 4.320 & 16 & .0536 & .0536 & 620 & 2.072 & 6 & .2330 & .2329 & 151 \\
\hline \multirow[b]{2}{*}{4.070} & \multirow[b]{2}{*}{8} & \multirow[b]{2}{*}{.0604} & $\int .0600$ & 900 & 1.9754 & 85 & .2563 & .2563 & 002 \\
\hline & & & $\left\{\begin{array}{l}.0605 \\
\text { S }\end{array}\right.$ & 030 & 1.93 & 22 & .2669 & .2669 & $18,2,0$ \\
\hline 3.952 & 236 & .0640 & .0641 & 001 & & \multirow[b]{2}{*}{8} & \multirow[b]{2}{*}{.2745} & .2000 & $\begin{array}{r}600 \\
154\end{array}$ \\
\hline \multirow{2}{*}{3.865} & \multirow{2}{*}{140} & \multirow{2}{*}{.0669} & $\{.0667$ & 910 & & & & $\left\{\begin{array}{l}27 \\
.2748\end{array}\right.$ & $12,5,0$ \\
\hline & & & 1.0672 & 330 & 1.8696 & 7 & .2861 & .2861 & 222 \\
\hline \multirow[t]{2}{*}{3.742} & \multirow[t]{2}{*}{7} & \multirow[t]{2}{*}{.0714} & $\left\{\begin{array}{l}0707 \\
.0708 \\
.0715\end{array}\right.$ & $\begin{array}{l}301 \\
011 \\
111\end{array}$ & 35 & 13 & .2895 & $\left\{\begin{array}{l}.2897 \\
.2898\end{array}\right.$ & $\begin{array}{l}612 \\
322\end{array}$ \\
\hline & & & & & 1.8528 & 15 & .2913 & .2913 & $15,3,1$ \\
\hline 3.682 & 8 & .0738 & .0738 & 211 & 1.8496 & 11 & .2923 & .2922 & 951 \\
\hline \multirow[t]{2}{*}{3.663} & \multirow[t]{2}{*}{19} & \multirow[t]{2}{*}{.0745} & $\{.0741$ & $10,0,0$ & \multirow{3}{*}{$\begin{array}{l}1.8239 \\
1.8195\end{array}$} & \multirow{3}{*}{$\begin{array}{l}25 \\
32\end{array}$} & \multirow{3}{*}{$\begin{array}{l}.3006 \\
.3021\end{array}$} & .3006 & $18,3,0$ \\
\hline & & & L.0743 & 820 & & & & .3021 & 960 \\
\hline 3.596 & 6 & .0774 & .0775 & 311 & & & & $\int .3230$ & 912 \\
\hline 3.562 & 10 & .0788 & .0790 & 530 & 1.7593 & 23 & .3231 & $\left\{\begin{array}{l}.3235 \\
\text { s }\end{array}\right.$ & 332 \\
\hline \multirow[t]{2}{*}{3.388} & 156 & .0871 & $\left\{\begin{array}{r}.0869 \\
0872\end{array}\right.$ & $\begin{array}{l}920 \\
630\end{array}$ & & & & (.3302 & 170 \\
\hline & & & 6.0872 & 630 & 1.7384 & 22 & .3309 & $\{.3310$ & $18,2,1$ \\
\hline 3.223 & 12 & .0962 & .0964 & $11,1,0$ & 1.7332 & 33 & .3329 & .3328 & 661 \\
\hline 3201 & 24 & 0078 & $\int .0975$ & 611 & & & & & \\
\hline 3.201 & 24 & .0976 & $\{.0976$ & 321 & $\begin{array}{l}1.7317 \\
1.7284\end{array}$ & $\begin{array}{l}35 \\
52\end{array}$ & $\begin{array}{l}.3335 \\
3348\end{array}$ & $\begin{array}{l}.3334 \\
.3348\end{array}$ & $\begin{array}{l}21,1,0 \\
15,5,0\end{array}$ \\
\hline 3.061 & 14 & .1067 & .1067 & $12,0,0$ & 1.7244 & 51 & $\begin{array}{l}.3540 \\
.3363\end{array}$ & .3362 & 370 \\
\hline 3.049 & 22 & .1076 & .1076 & 040 & & & & .3384 & $15,4,1$ \\
\hline 2.968 & 100 & .1135 & .1134 & 12,10 & 1.7184 & 8 & .3386 & $\left\{\begin{array}{l}.3389 \\
\text { s }\end{array}\right.$ & $12,5,1$ \\
\hline 2.959 & 106 & 0.1142 & 0.1143 & 340 & & & & & \\
\hline 2.917 & 10 & .1175 & .1176 & 621 & & & & $\int .3432$ & 922 \\
\hline $\begin{array}{l}2.880 \\
2.803\end{array}$ & 33 & .1205 & .1205 & 930 & 1.7072 & 22 & .3431 & $\{.3435$ & 631 \\
\hline & & & $\begin{array}{r}.1273 \\
.1308\end{array}$ & 911 & 1.6559 & 15 & .3647 & .3646 & $18,3,1$ \\
\hline 2.763 & 76 & .1310 & $\left\{\begin{array}{l}.1300 \\
.1313\end{array}\right.$ & 331 & 1.6524 & 25 & .3663 & .3662 & 961 \\
\hline & & & & & 1.6442 & 22 & 3699 & & $12,1,2$ \\
\hline 2.735 & 45 & .1337 & .1336 & $12,2,0$ & & & & 1.3705 & 342 \\
\hline 2.730 & 49 & .1342 & .1343 & $\begin{array}{r}12,0 \\
640\end{array}$ & 1.6294 & 8 & .3766 & .3768 & 932 \\
\hline 2.687 & 12 & .1385 & .1384 & 821 & & & & & \\
\hline 2.644 & 5 & .1431 & .1431 & 531 & & & & .3899 & $12,2,2$ \\
\hline 2.573 & 62 & .1511 & $\{.1510$ & 921 & 1.6012 & 12 & .3901 & $\{.3905$ & 642 \\
\hline & & & $\{.1513$ & 631 & 1.5926 & 5 & .3943 & .3943 & 171 \\
\hline 2.492 & 5 & .161 & .1609 & 731 & $\begin{array}{l}1.58 \\
1.58\end{array}$ & $\begin{array}{l}25 \\
32\end{array}$ & .3977 & .3975 & $21,1,1$ \\
\hline 2.421 & 6 & .1706 & .1708 & $12,0,1$ & 1.5801 & $\begin{array}{l}32 \\
24\end{array}$ & .3989 & .3989 & $15,5,1$ \\
\hline & 7 & .1716 & .1717 & 041 & & & .4005 & .4002 & 371 \\
\hline 2.373 & 49 & .1776 & .1775 & $12,1,1$ & 1.5654 & 5 & 4081 & 4081 & \\
\hline 2.368 & 59 & .1783 & :1783 & 341 & 1.5640 & 7 & .4089 & $\begin{array}{l}.4001 \\
.4088\end{array}$ & $\begin{array}{l}18,5,0 \\
15,6,0\end{array}$ \\
\hline
\end{tabular}

a Interplanar spacing.

b Observed intensity.

c Indexed on the basis of an orthorhombic unit cell with $a=36.740 \AA, b=12.195 \AA$ $c=3.951 \AA$.

\section{a. Room Temperature Monoclinic Polymorph}

A monoclinic polymorph of $\mathrm{WO}_{3}$ was reported by Tanisaki $\lceil 32\rceil$ to have a monoclinic unit cell with $a$ $=7.30 \AA, b=7.53 \AA, c=7.68 \AA, \beta=90^{\circ} 54^{\prime}$ at room temperature. However, the x-ray diffraction powder pattern can be completely indexed with the $c$-axis equal to one-half that of the true unit cell. The presence of superstructure requiring the doubled cell can apparently only be found with single crystal data. For this reason, the x-ray diffraction powder pattern listed in table 14 has been indexed on the basis of one-half the real $c$-axis value. The unit cell dimensions obtained from this pattern were found to be $a=7.299 \AA, b=7.535 \AA, c=7.688$ (3.844) $\AA, \beta$ $=90^{\circ} 54^{\prime}$. With 1 mole percent solid solution of $\mathrm{Nb}_{2} \mathrm{O}_{5}$ in $\mathrm{WO}_{3}$, the parameters are changed to $a$
$=7.317 \AA, b=7.532 \AA, c=7.684$ (3.842) $\AA, \beta=90^{\circ} 55^{\prime}$. For pure $\mathrm{WO}_{3}$ this monoclinic polymorph is apparently stable from about $17^{\circ} \mathrm{C}[32]$ to about $310^{\circ} \mathrm{C}$.

TABLE 12. X-ray diffraction powder data for the composition $\mathrm{Nb}_{2} \mathrm{O}_{5}: 11 \mathrm{WO}_{3}($ CuK radiation $)$

\begin{tabular}{l|r||r|r}
\hline \hline$d^{\mathrm{a}}$ & $I^{\mathrm{b}}$ & $d^{\mathrm{a}}$ & $I^{\mathrm{b}}$ \\
\hline & & & \\
4.638 & 5 & 1.927 & 90 \\
4.308 & 7 & 1.910 & 30 \\
3.966 & 350 & 1.865 & 58 \\
3.850 & 230 & 1.845 & 90 \\
3.726 & 350 & $1.812 \mathrm{~B}$ & 18 \\
& 15 & 1.712 & 32 \\
3.690 & 10 & 1.700 & 14 \\
$3.116 \mathrm{~B}$ & 178 & 1.677 & 50 \\
$2.764 \mathrm{~B}$ & 190 & 1.664 & 8 \\
2.677 & 28 & 1.569 & 18 \\
2.670 & 25 & 1.541 & 25 \\
2.579 & 25 & 1.530 & 10 \\
2.190 & 12 & $1.493 \mathrm{~B}$ & \\
2.139 & 10 & & \\
$1.998 \mathrm{~B}$ & & & \\
\hline
\end{tabular}

$\mathrm{B}=$ broad.

anterplaner spacing.

${ }^{\mathrm{b}}$ Observed intensity.

TABLE 13. X-ray diffraction powder data for the composition $\mathrm{Nb}_{2} \mathrm{O}_{5}: 15 \mathrm{WO}_{3}\left(C u K_{a}\right.$ radiation $)$

\begin{tabular}{l|r||c|c}
\hline \hline$d^{\mathrm{a}}$ & $I^{\mathrm{b}}$ & $d^{\mathrm{a}}$ & $I^{\mathrm{b}}$ \\
\hline & & & \\
3.931 & 25 & 1.898 & 27 \\
3.839 & 284 & 1.869 & 65 \\
3.760 & 116 & 1.855 & 20 \\
3.682 & 336 & 1.841 & 40 \\
3.134 & 10 & $1.812 \mathrm{~B}$ & 15 \\
& & & 25 \\
3.089 & 23 & 1.799 & 23 \\
2.763 & 7 & 1.711 & 20 \\
2.676 & 140 & 1.695 & 65 \\
2.647 & 96 & $1.671 \mathrm{~B}$ & 15 \\
2.584 & 18 & 1.652 & 8 \\
2.214 & 14 & 1.573 & 10 \\
2.181 & 30 & 1.555 & 15 \\
2.152 & 12 & 1.546 & 34 \\
$2.006 \mathrm{~B}$ & 10 & 1.529 & 30 \\
1.922 & 58 & 1.525 & \\
& & & \\
\hline
\end{tabular}

$\mathrm{B}=$ broad.

anterplaner spacing.

${ }^{\mathrm{b}}$ Observed intensity.

\section{b. Low-Temperature Triclinic Polymorph}

According to Tanisaki [32] the room temperature polymorph of $\mathrm{WO}_{3}$ transforms to a triclinic form at about $17{ }^{\circ} \mathrm{C}$, on cooling. However, on reheating there is still some of the triclinic form remaining at room temperature, indicating some hysteresis in the phase transition. It was found in the present study, that the room temperature monoclinic polymorph could be partially transformed to the triclinic modification by grinding the specimen in a mortar and pestle, without lowering the temperature. The unit cell dimensions of the triclinic form were reported [32] as $a=7.30 \AA, b=7.52 \AA, c=7.69 \AA, \alpha=88^{\circ} 50^{\prime}$, $\beta=90^{\circ} 55^{\prime}, \gamma=90^{\circ} 56^{\prime}$. The indexed x-ray diffraction 
TABLE 14. X-ray diffraction powder data for the room temperature (monoclinic) form of $\mathrm{WO}_{3}($ CuK $a$ radiation $)$

\begin{tabular}{|c|c|c|c|c|c|c|c|c|c|}
\hline$d^{\mathrm{a}}$ & $I^{\mathrm{b}}$ & $\frac{1}{d^{2}}$ obs & $\frac{1}{d^{2}}$ calc & $h k^{\prime}{ }^{c}$ & $d^{\mathrm{a}}$ & $I^{\mathrm{b}}$ & $\frac{1}{d^{2}}$ obs & $\frac{1}{d^{2}}$ calc & $h k^{\prime c}$ \\
\hline 3.840 & 154 & 0.0678 & 0.0677 & 001 & 1.8111 & 45 & 0.3049 & 0.3050 & $11 \overline{2}$ \\
\hline 3.761 & 160 & .0707 & .0705 & 020 & 1.7977 & 42 & .3094 & .3094 & 112 \\
\hline 3.646 & 268 & .0752 & .0751 & 200 & & & & 3413 & 022 \\
\hline 3.419 & 5 & .0856 & .0853 & 011 & 1.7116 & 48 & .3414 & $\left\{\begin{array}{r}.5415 \\
345\end{array}\right.$ & 022 \\
\hline 3.344 & 65 & .0894 & .0892 & 120 & & & & (.3414 & 202 \\
\hline 3.114 & 70 & .1031 & .1030 & $11 \overline{1}$ & 1.6914 & 40 & .3495 & .3495 & 041 \\
\hline 3.083 & 50 & .1052 & .1052 & 111 & 1.6894 & 32 & .3504 & .3504 & 202 \\
\hline 2.689 & 132 & .1383 & .1382 & 021 & & & & & \\
\hline 2.667 & 72 & .1407 & .1406 & 210 & 1.6740 & 35 & $\begin{array}{l}.3569 \\
3627\end{array}$ & .3569 & 240 \\
\hline 2.629 & 54 & .1447 & .1450 & $20 \overline{1}$ & $\begin{array}{l}1.6581 \\
1.6504\end{array}$ & $\begin{array}{l}34 \\
24\end{array}$ & $\begin{array}{l}.3637 \\
3671\end{array}$ & $\begin{array}{l}3636 \\
.3672\end{array}$ & $40 \frac{1}{14}$ \\
\hline & & & & & 1.6455 & 42 & .3693 & .3694 & 141 \\
\hline 2.620 & 152 & .1457 & .1455 & 220 & 1.6423 & 80 & .3708 & .3708 & 420 \\
\hline $\begin{array}{l}2.533 \\
2.514\end{array}$ & $\begin{array}{l}22 \\
27\end{array}$ & $\begin{array}{l}.1559 \\
.1582\end{array}$ & $\begin{array}{l}.1558 \\
.1580\end{array}$ & $\begin{array}{l}121 \\
121\end{array}$ & & & & & \\
\hline 2.176 & 38 & .2112 & .2110 & 221 & 1.6385 & 50 & .3725 & .3725 & 401 \\
\hline \multirow[t]{2}{*}{2.154} & 36 & .2156 & .2155 & $\begin{array}{l}221 \\
221\end{array}$ & 1.6231 & 5 & .3919 & .3918 & $33 \overline{1}$ \\
\hline & & & & 221 & 1.5843 & 5 & .3984 & .3985 & 331 \\
\hline 2.103 & & 2262 & 2262 & & 1.5584 & 30 & .4117 & .4119 & $22 \overline{2}$ \\
\hline 2.043 & 17 & 2395 & .2304 & 031 & 1.5419 & 32 & .4206 & .4208 & 222 \\
\hline 2.025 & 12 & .2438 & 2439 & & & & & & \\
\hline 2.016 & 14 & 2461 & 2461 & 131 & 1.5384 & 27 & .4225 & .4224 & $24 \overline{1}$ \\
\hline \multirow{2}{*}{1.9955} & 20 & 2511 & $\begin{array}{r}2401 \\
2509\end{array}$ & $13 \frac{1}{1}$ & 1.5312 & 10 & .4265 & -4268 & $24 \underline{1}$ \\
\hline & & & & 311 & 1.5179 & 15 & .4341 & .4341 & $42 \overline{1}$ \\
\hline 1.9709 & 12 & 2574 & & & 1.5029 & 20 & .4427 & .4430 & 421 \\
\hline 1.9216 & 44 & 2708 & 2708 & 311 & 1.4981 & 22 & .4456 & .4459 & 132 \\
\hline 1.8838 & 60 & .2818 & & 002 & & & & & \\
\hline \multirow{3}{*}{1.8246} & \multirow{3}{*}{133} & \multirow{3}{*}{.3004} & & & 14899 & 42 & 4505 & .4500 & $31 \frac{2}{2}$ \\
\hline & & & $\left\{\begin{array}{r}.5007 \\
3006\end{array}\right.$ & 400 & & & & .4508 & 340 \\
\hline & & & & & 1.4679 & 14 & .4641 & .4641 & 312 \\
\hline
\end{tabular}

a Interplaner spacing.

Indexed on the basis of a monoclinic unit cell with $a=7.299 \AA, b=7.535 \AA, c=3.844 \AA$ $\left(\frac{1}{2} \times 7.688 \AA\right), \beta=90^{\circ} 54$

powder pattern of the triclinic polymorph obtained at room temperature from a specimen which had been removed from liquid nitrogen, is listed in table 15 . The value of the $c$-axis was again halved, as the larger value is not necessary to index the powder pattern. According to Tanisaki, this phase is stable from about $-40{ }^{\circ} \mathrm{C}$ to about $17{ }^{\circ} \mathrm{C}$. The triclinic polymorph was never observed in any of the $\mathrm{Nb}_{2} \mathrm{O}_{5}-\mathrm{WO}_{3}$ solid solutions.
From about $-40{ }^{\circ} \mathrm{C}$ to below liquid nitrogen temperature, another monoclinic polymorph occurs in pure $\mathrm{WO}_{3}$ which was reported by Tanisaki [32] to have $a=5.27 \AA, b=5.16 \AA, c=7.67 \AA, \beta=91^{\circ} 43^{\prime}$. This polymorph can be obtained essentially single phase at room temperature by quenching a solid solution of $2 \mathrm{Nb}_{2} \mathrm{O}_{5}: 98 \mathrm{WO}_{3}$ from the temperature interval $1230{ }^{\circ} \mathrm{C}$ to about $1385{ }^{\circ} \mathrm{C}$, the solidus temperature. This polymorph also occurs as a mixture with other polymorphs in specimens containing 1 and 3 mole percent $\mathrm{Nb}_{2} \mathrm{O}_{5}$. The unit cell dimensions obtained from the powder pattern for the 2 mole percent $\mathrm{Nb}_{2} \mathrm{O}_{5}$ specimen are $a=5.305 \AA, b=5.192 \AA$. $c=7.671 \mathrm{~A}, \beta=91^{\circ} 33^{\prime}$. The indexed x-ray diffraction powder pattern is given in table 16 , utilizing the $7.671 \AA$ value for $c$ as several peaks necessitating this doubled value are observed in the powder pattern. Hightemperature $\mathrm{x}$-ray patterns show this phase to be apparently stable in the 2 mole percent $\mathrm{Nb}_{2} \mathrm{O}_{5}$ composition up to a temperature of about $735^{\circ} \mathrm{C}$, at which temperature it transforms to a tetragonal polymorph. If the temperature is not raised above about $750{ }^{\circ} \mathrm{C}$, this transition is reversible. However, if the temperature is raised to $900{ }^{\circ} \mathrm{C}$ the low-temperature monoclinic polymorph is not recovered and the tetragonal phase transforms to the orthorhombic polymorph.

Tungsten trioxide has been reported to be ferroelectric below about $-40{ }^{\circ} \mathrm{C}$ [33]. Specimens of sintered $\mathrm{WO}_{3}$ containing 2 to 4 mole percent $\mathrm{Ta}_{2} \mathrm{O}_{5}$ were reported to exhibit ferroelectric behavior at room temperature [34]. The low-temperature monoclinic polymorph formed by quenching a specimen containing 2 mole percent $\mathrm{Nb}_{2} \mathrm{O}_{5}$ was examined for evidence of ferroelectricity [35]. However, no conclusive ferroelectric properties could be found.

TABLE 15. X-ray diffraction powder data for the triclinic form of $\mathrm{W} \mathrm{O}_{3}\left(C u K_{\text {" }}\right.$ radiation $)$

\begin{tabular}{|c|c|c|c|c|c|c|c|c|c|c|c|c|c|c|}
\hline$d^{\mathrm{a}}$ & $I^{\mathrm{b}}$ & $\frac{1}{d^{2}}$ obs & $\frac{1}{d^{2}}$ calc & $h k l^{c}$ & $d^{\mathrm{a}}$ & $I^{\mathrm{b}}$ & $\frac{1}{d^{2}}$ obs & $\frac{1}{d^{2}}$ calc & $h k l^{c}$ & $d^{\mathrm{a}}$ & $I^{\mathrm{b}}$ & $\frac{1}{d^{2}}$ obs & $\frac{1}{d^{2}}$ calc & $h h^{\prime c}$ \\
\hline 3.840 & 284 & 0.0678 & 0.0677 & 001 & 2.159 & 20 & 0.2145 & 0.2145 & $\overline{2} 21$ & 1.8040 & 26 & 0.3073 & 0.3068 & $\overline{1} 12$ \\
\hline 3.760 & 224 & .0707 & .0707 & 020 & 2.154 & 53 & .2155 & .2158 & 221 & 1.8007 & 43 & .3084 & .3086 & $1 \overline{1} 2$ \\
\hline 3.652 & 350 & .0750 & .0749 & 200 & 2.150 & 51 & .2163 & .2163 & 221 & 1.7931 & 26 & .3110 & .3113 & $11 \overline{2}$ \\
\hline 3.362 & 20 & .0885 & .0885 & 120 & 2.12 & 3 & .2224 & .2227 & $03 \overline{1}$ & 1.7244 & 17 & .3363 & .3360 & $02 \overline{2}$ \\
\hline 3.322 & 25 & .0906 & .0906 & 120 & 2.082 & 5 & .2306 & .2310 & 031 & 1.7113 & 24 & .3415 & .3420 & $20 \overline{2}$ \\
\hline 3.143 & 42 & .1012 & .1013 & $\overline{111}$ & 2.059 & 4 & .2358 & .2360 & $\underline{\overline{3} 20}$ & 1.7013 & 23 & .3455 & $\left\{\begin{array}{l}.3451 \\
.3457\end{array}\right.$ & $\begin{array}{l}041 \\
202\end{array}$ \\
\hline 3.099 & 20 & .1041 & .1042 & $\overline{1} 11$ & 2.046 & 8 & .2388 & .2389 & 131 & 16975 & 22 & .3470 & $\begin{array}{r}.3457 \\
.3470\end{array}$ & $\begin{array}{l}202 \\
022\end{array}$ \\
\hline 3.085 & 65 & .1051 & .1051 & $1 \overline{1} 1$ & 2.032 & 5 & .2422 & .2423 & 320 & $\begin{array}{l}1.0915 \\
1.6897\end{array}$ & 17 & .3503 & .3506 & 041 \\
\hline 3.070 & 42 & .1061 & .1059 & $11 \overline{1}$ & 2.025 & 10 & .2439 & .2439 & $\frac{131}{211}$ & $\begin{array}{l}1.0091 \\
1.6825\end{array}$ & 13 & .3533 & .3536 & $\underline{240}$ \\
\hline 2.714 & 55 & .1359 & .1358 & $02 \overline{1}$ & 2.011 & 9 & .2474 & .2481 & $\overline{311}$ & 1.6653 & 16 & .3606 & .3608 & $\overline{141}$ \\
\hline 2.667 & 108 & .1407 & .1407 & $20 \overline{1}$ & 2.005 & 12 & .2487 & .2490 & $13 \overline{1}$ & & & & & \\
\hline 2.660 & 102 & .1413 & .1413 & 021 & 1.9985 & 10 & .2504 & .2503 & $1 \overline{3} 1$ & 1.6612 & 52 & .3624 & .3620 & $24 \underline{0}$ \\
\hline 2.640 & 80 & .1435 & .1435 & $\overline{2} 20$ & 1.9844 & 9 & .2540 & .2540 & $3 \overline{1} 1$ & 1.6570 & 31 & .3642 & .3635 & $40 \overline{1}$ \\
\hline 2.632 & 93 & .1443 & .1443 & 201 & 1.9746 & 12 & .2565 & .2564 & $\frac{3}{2} 1 \overline{1}$ & 1.6537 & 28 & .3657 & .3660 & $\underline{4} 20$ \\
\hline 2.600 & 58 & .1480 & .1477 & 220 & 1.9713 & 14 & .2573 & .2568 & 311 & 1.6502 & 33 & .3672 & $\{.3669$ & $\overline{1} 41$ \\
\hline 2.562 & 13 & .1524 & .1524 & $\overline{121}$ & 1.9216 & 48 & .2708 & .2708 & 002 & 16358 & 30 & .3738 & $\begin{array}{l}.3672 \\
.3736\end{array}$ & 401 \\
\hline 2.527 & 11 & .1567 & .1564 & 121 & 1.8801 & 52 & .2829 & .2829 & 040 & & & & & \\
\hline $\begin{array}{l}2.501 \\
2.500\end{array}$ & $\begin{array}{l}15 \\
17\end{array}$ & $\begin{array}{l}.1598 \\
.1600\end{array}$ & $\begin{array}{l}.1598 \\
.1600\end{array}$ & 121 & 1.8273 & 148 & .2995 & $\left\{\begin{array}{l}.2995 \\
2995\end{array}\right.$ & $\frac{400}{140}$ & 1.6337 & 35 & .3747 & .3744 & 420 \\
\hline 2.202 & 20 & .2063 & .2066 & $\overline{221}$ & 1.8198 & 44 & .3020 & .3021 & 112 & 1.6303 & 18 & .3763 & .3760 & 141 \\
\hline
\end{tabular}

a Interplaner spacing.

b Observed intensity.

' Indexed on the basis of a triclinic unit cell with $a=7.30 \AA, b=7.52 \AA, c=3.845 \AA\left(\frac{1}{2} \times 7.69 \AA\right), \alpha=88^{\circ} 50^{\prime}, \beta=90^{\circ} 55^{\prime}, \gamma=90^{\circ} 56^{\prime}$. 
TABLE 16. X-ray diffraction powder data for the low-temperature monoclinic form of $\mathrm{WO}_{3}$ taken from $2 \mathrm{Nb}_{2} \mathrm{O}_{5}: 98 \mathrm{WO}_{3}$ solid solution $(\mathrm{CuK}$ a radiation $)$

\begin{tabular}{|c|c|c|c|c|c|c|c|c|c|}
\hline$d^{\text {a }}$ & $I^{\mathrm{b}}$ & $\frac{1}{d^{2}}$ obs & $\frac{1}{d^{2}}$ calc & $h k l^{\mathrm{C}}$ & $d^{\text {a }}$ & $I^{\mathrm{b}}$ & $\frac{1}{d^{2}}$ obs & $\frac{1}{d^{2}}$ calc & $h k l^{\mathrm{C}}$ \\
\hline 3.834 & 400 & 0.0680 & 0.0680 & 002 & 1.7990 & 54 & 0.3090 & 0.3090 & 014 \\
\hline 3.703 & 380 & .0729 & .0729 & 110 & 1.7875 & 28 & .3130 & .3130 & 104 \\
\hline 3.145 & 25 & .1011 & 1011 & $10 \overline{2}$ & $1.7163 \mid$ & 37 & . 3395 & .3394 & $11 \overline{4}$ \\
\hline 3.083 & 50 & .1052 & .1051 & 012 & 1.6900 & 25 & .3501 & .3501 & 114 \\
\hline 3.067 & 32 & .1063 & .1064 & 102 & 1.6805 & 37 & .3541 & .3542 & $22 \overline{2}$ \\
\hline 2.691 & 138 & . 1381 & .1382 & 112 & 1.6692 & 41 & 列. .3589 & . 3592 & 310 \\
\hline 2.641 & 144 & . 1433 & $\left\{\begin{array}{l}1432 \\
i 145\end{array}\right.$ & 200 & 1.6557 & 28 & . 3648 & . 3648 & 222 \\
\hline 2.594 & 40 & .1486 & $\left\{\begin{array}{l}\{.1435 \\
1484\end{array}\right.$ & 112 & 1.6448 & 26 & .3697 & .3696 & 130 \\
\hline 2.458 & 6 & .1655 & $\begin{array}{l}.1484 \\
1654\end{array}$ & 020 . & 1.6179 & 4 & . 3820 & . 3821 & 302 \\
\hline 2.294 & 10 & .1900 & $\begin{array}{l}.1054 \\
.1900\end{array}$ & $\begin{array}{l}021 \\
013\end{array}$ & 1.5851 & 4 & 年. .3900 & . 3981 & 302 \\
\hline & & & & & 1.5776 & 5 & .4018 & .4018 & 032 \\
\hline 2.236 & 5 & .2000 & $\left\{\begin{array}{l}.1998 \\
1000\end{array}\right.$ & 121 & 1.5729 & 10 & . 4042 & . 4044 & $20 \overline{4}$ \\
\hline 2206 & 22 & 2056 & {$[.1999$} & 211 & 1.5464 & 18 & .4195 & .4192 & $31 \overline{2}$ \\
\hline 2.200 & 22 & .2006 & $\begin{array}{r}2058 \\
2163\end{array}$ & 202 & 1.5422 & 21 & .4205 & .4202 & 024 \\
\hline 2.148 & 70 & .2167 & $\left\{\begin{array}{l}.2163 \\
.2165\end{array}\right.$ & $\begin{array}{l}022 \\
202\end{array}$ & 1.5326 & 6 & .4257 & . 4257 & 204 \\
\hline 2.120 & 12 & .2429 & .2429 & $21 \overline{2}$ & & & & $\int .4349$ & $13 \overline{2}$ \\
\hline 2.002 & 14 & .2496 & .2495 & $12 \overline{2}$ & 1.5161 & 28 & .4351 & $\{.4352$ & 312 \\
\hline 1.9860 & 17 & .2535 & . 2536 & 212 & 1.5056 & 26. & .4412 & $\left\{\begin{array}{c}.4402 \\
4445\end{array}\right.$ & $\begin{array}{l}132 \\
214\end{array}$ \\
\hline 1.9815 & 18 & .2547 & .2548 & 122 & 14895 & 13 & .4508 & $\begin{array}{l}-.4415 \\
4507\end{array}$ & $\begin{array}{l}214 \\
124\end{array}$ \\
\hline 1.9178 & 68 & 2719 & .2719 & 004 & 1.4895 & 15 & & $\int .4614$ & 124 \\
\hline 1.8521 & 100 & 2915 & 2915 & 220 & 1.4700 & 24 & .4621 & $\{.4628$ & 214 \\
\hline 1.8192 & 27 & .3022 & .3023 & $10 \overline{4}$ & & & & & \\
\hline
\end{tabular}

a Interplaner spacing.

${ }^{\mathrm{b}}$ Observed intensity.

c Indexed on the basis of a monoclinic unit cell with $a=5.305 \AA . b=5.192 \AA, c=7.671 \AA$, $\beta=91^{\circ} 33^{\prime}$.

\section{d. High-Temperature Orthorhombic Polymorph}

Although no thermal effect was indicated with DTA, the room-temperature monoclinic polymorph of pure $\mathrm{WO}_{3}$ is observed in the high temperature $\mathrm{x}$-ray furnace to transform reversibly at $310{ }^{\circ} \mathrm{C}$ to an orthorhombic phase in agreement with Wyart and Foex [31]. This orthorhombic form is observed at room temperature in the solid solutions whenever the low-temperature monoclinic polymorph is heated to about $900{ }^{\circ} \mathrm{C}$ and cooled by removing from the furnace. The indexed x-ray diffraction powder pattern of a specimen of this polymorph containing 2 mole percent $\mathrm{Nb}_{2} \mathrm{O}_{5}$ is listed in table 17. It shows no indication of a doubled $c$-axis and is indexed on the basis of the smaller orthorhombic cell. The unit cell dimensions obtained for this composition, at room temperature, are $a=7.384 \AA, b=7.512 \AA, c=7.692(3.846) \AA$. The orthorhombic phase is observed in this composition to transform to tetragonal in the high temperature x-ray furnace at $510{ }^{\circ} \mathrm{C}$ and the reverse transition was observed to take place at $440{ }^{\circ} \mathrm{C}$.

\section{e. Tetragonal High-Temperature Polymorph(s)}

In pure $\mathrm{WO}_{3}$ the orthorhombic polymorph was found to undergo a transition to the tetragonal form at about $750{ }^{\circ} \mathrm{C}$ in the high-temperature $\mathrm{x}$-ray furnace. The DTA data indicated that the phase transition occurred at a temperature of $740{ }^{\circ} \mathrm{C}$ on heating and $730{ }^{\circ} \mathrm{C}$ on cooling. These results are in reasonable agreement with those of previous workers [7, 31]. The tetragonal polymorph is found as a single phase as low as $440{ }^{\circ} \mathrm{C}$ in the specimen containing 2 mole percent $\mathrm{Nb}_{2} \mathrm{O}_{5}$. However, in the 3 mole percent $\mathrm{Nb}_{2} \mathrm{O}_{5}$ specimen, the low-temperature monoclinic polymorph transforms after heating at about $900{ }^{\circ} \mathrm{C}$ into a mixture consisting predominantly of a tetragonal modification with only a trace of the orthorhombic form. The unit cell dimensions obtained at room temperature from this composition are $a=5.265 \AA$, $c=3.846 \AA$, and the $\mathrm{x}$-ray diffraction powder pattern from which the data were obtained, indexed on the basis of the smaller cell, is listed in table 18.

In addition to the transformation at $730-740{ }^{\circ} \mathrm{C}$, another transition is observed at about $900{ }^{\circ} \mathrm{C}$ in pure $\mathrm{WO}_{3}$, as indicated by a very small peak in the DTA pattern. High-temperature x-ray diffraction powder patterns indicate that $\mathrm{WO}_{3}$ is tetragonal both above and below this minor thermal discontinuity. It is possible that the unit cell has a doubled $c$-axes below $900{ }^{\circ} \mathrm{C}$ and only above this temperature does the powder pattern yield the correct unit cell. However, this hypothesis cannot be verified without single crystal data.

Although $\mathrm{WO}_{3}$ might be expected to have a cubic polymorph of the $\mathrm{ReO}_{3}$-type, there is no evidence in either the present work or previously reported studies that such a polymorph exists.

TABLE 17. X-ray diffraction powder data for the orthorhombic form of $\mathrm{WO}_{3}$ taken from $2 \mathrm{Nb}_{2} \mathrm{O}_{5}: 98 \mathrm{WO}_{3}$ solid solution $\left(C u K_{a}\right.$ radiation)

\begin{tabular}{|c|c|c|c|c|}
\hline$d^{\mathrm{a}}$ & $I^{\mathrm{b}}$ & $\frac{1}{d^{d}}$ obs & $\frac{1}{d^{2}}$ calc & $h k l^{\mathrm{c}}$ \\
\hline 3.845 & 248 & 0.0676 & 0.0676 & 001 \\
\hline & 156 & .0710 & .0709 & 020 \\
\hline 3.691 & 232 & .0734 & .0734 & 200 \\
\hline 3.427 & 5 & .0852 & .0853 & 011 \\
\hline 3.346 & 18 & .0893 & .0892 & 120 \\
\hline 3.104 & 60 & . 1038 & .1037 & 111 \\
\hline & 85 & . 1386 & .1385 & 021 \\
\hline 2.662 & 80 & .1411 & .1409 & 201 \\
\hline 2.633 & 126 & .1443 & .1442 & 220 \\
\hline 2.525 & 10 & .1568 & .1568 & 121 \\
\hline 2.173 & 42 & .2119 & .2118 & 221 \\
\hline & 5 & .2270 & & 031 \\
\hline 2.05 & 5 & .2365 & .2359 & 320 \\
\hline 2.018 & 20 & .2455 & .2454 & 131 \\
\hline 1.9976 & 14 & .2506 & .2504 & 311 \\
\hline 1.9232 & 48 & .2704 & .2704 & 002 \\
\hline 1.87 & 33 & .283 & .283 & 040 \\
\hline & 46 & .293 & & 400 \\
\hline & 21 & .3020 & .30 & 140 \\
\hline 1.8064 & 37 & .3065 & .3064 & 112 \\
\hline 1.7119 & 24 & .3412 & .3413 & 022 \\
\hline & 2 & & & \\
\hline & 3 & .35 & .35 & 041 \\
\hline & 26 & .3569 & .356 & 240 \\
\hline 1.6645 & 32 & .3609 & .3610 & 401 \\
\hline 1.6568 & 34 & .3643 & .3643 & 420 \\
\hline 1.645 & 14 & & & 141 \\
\hline 1.59 & 5 & & .392 & 331 \\
\hline 1.553 & 10 & .4147 & .4146 & 222 \\
\hline & 17 & .4244 & .4245 & 241 \\
\hline 1.5217 & 20 & .4319 & .4319 & 421 \\
\hline 1.4933 & 16 & .4484 & $\left\{\begin{array}{l}4482 \\
4486\end{array}\right.$ & \\
\hline 1.4858 & 13 & .4530 & .4531 & $\begin{array}{l}340 \\
312\end{array}$ \\
\hline
\end{tabular}

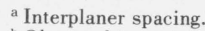

Observed intensity.

c Indexed on the basis of an orthorhombic unit cell with $a=7.384 \AA, b=7.512 \AA, c=3.846$ $\AA\left(\frac{1}{2} \times 7.692 \AA\right)$. 
TABLE 18. X-ray diffraction powder data for the tetragonal form of $\mathrm{WO}_{3}$ taken from $3 \mathrm{Nb}_{2} \mathrm{O}_{5}: 97 \mathrm{WO}_{3}$ solid solution $\left(C u K_{\alpha}\right.$ radiation)

\begin{tabular}{l|r|r|r|r}
\hline \hline$d^{\mathrm{a}}$ & \multicolumn{1}{|c|}{$I^{\mathrm{b}}$} & $\frac{1}{d^{2}} \mathrm{obs}$ & $\frac{1}{d^{2}}$ calc & $h k l^{\mathrm{c}}$ \\
& & & & \\
\hline & & & & \\
3.847 & 280 & 0.0676 & 0.0676 & 001 \\
3.725 & 252 & .0721 & .0722 & 110 \\
3.106 & 58 & .1037 & .1037 & 101 \\
2.674 & 116 & .1398 & .1398 & 111 \\
2.638 & 96 & .1438 & .1443 & 200 \\
& & & & \\
2.173 & 40 & .2118 & .2119 & 201 \\
2.008 & 25 & .2481 & .2480 & 211 \\
1.923 & 45 & .2705 & .2705 & 002 \\
1.861 & 48 & .2886 & .2886 & 220 \\
1.806 & 42 & .3067 & .3066 & 102 \\
& & & & \\
1.707 & 26 & .3430 & .3426 & 112 \\
1.675 & 40 & .3566 & .3563 & 201 \\
1.665 & 48 & .3606 & .3608 & 310 \\
1.597 & 5 & .3922 & .3923 & 301 \\
1.552 & 8 & .4150 & .4148 & 202 \\
1.528 & 28 & .4284 & .4284 & 311 \\
1.489 & 15 & .4510 & .4509 & 212 \\
\hline
\end{tabular}

a Interplanar spacing.

Indexed on the basis of a tetragonal unit cell with $a=5.265 \AA, c=3.846 \AA$.

\section{Discussion of Phase Equilibria}

The postulated phase equilibrium diagram of the binary system $\mathrm{Nb}_{2} \mathrm{O}_{5}-\mathrm{WO}_{3}$ is shown in figure 1 . The experimental data from which this diagram was constructed are given in table 19 . The system contains four compounds which are shown as melting congruently, $\quad 6 \mathrm{Nb}_{2} \mathrm{O}_{5} \cdot \mathrm{WO}_{3}, \quad " \quad 6 \mathrm{Nb}_{2} \mathrm{O}_{5} \cdot 11 \mathrm{~W} \mathrm{O}_{3}$,", $4 \mathrm{Nb}_{2} \mathrm{O}_{5} \cdot 9 \mathrm{WO}_{3}$, and $2 \mathrm{Nb}_{2} \mathrm{O}_{5} \cdot 7 \mathrm{WO}_{3}$. Six compounds melt incongruently " $30 \mathrm{Nb}_{2} \mathrm{O}_{5} \cdot \mathrm{WO}_{3}, " 7 \mathrm{Nb}_{2} \mathrm{O}_{5} \cdot 3 \mathrm{WO}_{3}$, $8 \mathrm{Nb}_{2} \mathrm{O}_{5} \cdot 5 \mathrm{WO}_{3}, 9 \mathrm{Nb}_{2} \mathrm{O}_{5} \cdot 8 \mathrm{WO}_{3}$, and the two Magneli phases estimated to be " $\mathrm{Nb}_{2} \mathrm{O}_{5} \cdot 11 \mathrm{WO}_{3}$ " and " $\mathrm{Nb}_{2} \mathrm{O}_{5} \cdot 15 \mathrm{WO}_{3} . "$ In addition, two stable phases, $13 \mathrm{Nb}_{2} \mathrm{O}_{5} \cdot 4 \mathrm{WO}_{3}$ and $\mathrm{Nb}_{2} \mathrm{O}_{5} \cdot \mathrm{WO}_{3}$ dissociate before melting as does the apparently metastable disordered bronze type solid solution labeled " $3: 8$ " in the phase diagram. Six of the compounds $(8: 5,9: 8$, "6:11", $2: 7, " 1: 11, "$ and " $1: 15$ ") are shown on the phase diagram as having minimum stability temperatures. In many of these cases, it is impossible to determine whether or not the minimum temperature represents a stable transition. The existence of solid solution could neither be definitely established in any of the compounds nor in $\mathrm{Nb}_{2} \mathrm{O}_{5}$. However, $\mathrm{WO}_{3}$ accepts a maximum of about 3 mole percent $\mathrm{Nb}_{2} \mathrm{O}_{5}$ in solid solution.

The solidus temperature between the " $30: 1$ " phase and $6 \mathrm{Nb}_{2} \mathrm{O}_{5} \cdot \mathrm{WO}_{3}$ has been found to be about $1464{ }^{\circ} \mathrm{C}$. The exact eutectic composition has not been determined but probably exists between about 7 and 10 mole percent $\mathrm{WO}_{3}$. The peritectic corresponding to the incongruent melting point of $7 \mathrm{Nb}_{2} \mathrm{O}_{5} \cdot 3 \mathrm{WO}_{3}$ occurs at $1440{ }^{\circ} \mathrm{C}$ and about 40 mole percent $\mathrm{WO}_{3}$. However, the composition of the two peritectics at 1385 and $1375{ }^{\circ} \mathrm{C}$ and of the eutectic at $1364{ }^{\circ} \mathrm{C}$, were not determined exactly. They probably occur between about 60 and 63 mole percent $\mathrm{WO}_{3}$.

Both the 8:5 and 9:8 compounds apparently have minimum temperatures of stability. However, all efforts to locate accurately the temperature of these minimums proved inconclusive (table 19). For both compounds, the experimental temperature at which the phase begins to form on heating and the temperature at which it begins to dissociate on cooling had a wide range of overlap. Furthermore, the actual temperature of the minimum stability seemed to be strongly dependent on the purity content (table 19, parts I and II). For these reasons the minimum stability temperatures of the $8: 5$ and $9: 8$ are indicated on the phase diagram by dashed lines and the temperatures assigned to each, $\sim 1090$ and $\sim 1265{ }^{\circ} \mathrm{C}$, respectively, represent the best compromise between the inconsistencies in the experimental data.

None of the ordered bronze-type phases can be prepared in reasonable laboratory time at $1100{ }^{\circ} \mathrm{C}$. The "6:11" phase was not found to be well crystallized below about $1210{ }^{\circ} \mathrm{C}$. The minimum line at this temperature is dashed to indicate uncertainty in both the temperature value and the stability relation. The same discussion also is relative for the minimum value of the $2: 7$. In this case, however, the superstructure lines characteristic of this phase do not begin to appear below about $1245{ }^{\circ} \mathrm{C}$. The three ordered bronze-type phases, " $6: 11$ ", $4: 9$, and $2: 7$, all appear to melt congruently at 1378,1380 and 1357 ${ }^{\circ} \mathrm{C}$, respectively. The solidus temperatures between these phases at 1365 and $1335^{\circ} \mathrm{C}$ appear to be measurably lower than the observed melting temperature of the compounds. However, no eutectic compositions could be experimentally determined, possibly due to reduction and, therefore, the liquidus curves are necessarily dashed (see discussion on reduction in sec. 5).

The ability to distinguish a two-phase region between the two bronze-type phases $4 \mathrm{Nb}_{2} \mathrm{O}_{5} \cdot 9 \mathrm{WO}_{3}$ and $2 \mathrm{Nb}_{2} \mathrm{O}_{5}$ - $7 \mathrm{WO}_{3}$ was greatly increased by a separation of those phases in the experiments of long-time duration. The top of the specimens within the two-phase region showed only $4: 9$ in the $x$-ray diffraction pattern while the bottom of the specimen showed mostly $2: 7$. This apparent gravity separation is probably enhanced by an appreciable vapor phase in the sealed $\mathrm{Pt}$ tube and is considered to be due mainly to vapor transport. A similar gravity separation takes place throughout most of the system and adds more evidence to the probability of a two-phase region between " $6 \mathrm{Nb}_{2} \mathrm{O}_{5}$ $\cdot 11 \mathrm{WO}_{3}$ " and $4 \mathrm{Nb}_{2} \mathrm{O}_{5} \cdot 9 \mathrm{WO}_{3}$. This two-phase region is based mostly on the different morphology and color of these phases, as well as the slight differences in the superstructure exhibited by single crystal patterns. Almost all of the high-temperature phases in the $\mathrm{Nb}_{2} \mathrm{O}_{5}-\mathrm{WO}_{3}$ system are needle formers. Most of those which contain appreciable $\mathrm{WO}_{3}$ are yellowgreen or blue-green in color. However, the "6:11" phase is white and forms tabular crystals rather than needles.

A eutectic exists between the 2:7 compound and the Magneli "shear" phases at about $1340{ }^{\circ} \mathrm{C}$ and 83 mole percent $\mathrm{WO}_{3}$. However, the exact compositions of the peritectics at 1356 and $1358{ }^{\circ} \mathrm{C}$, corresponding to the decomposition temperatures have not been determined. 


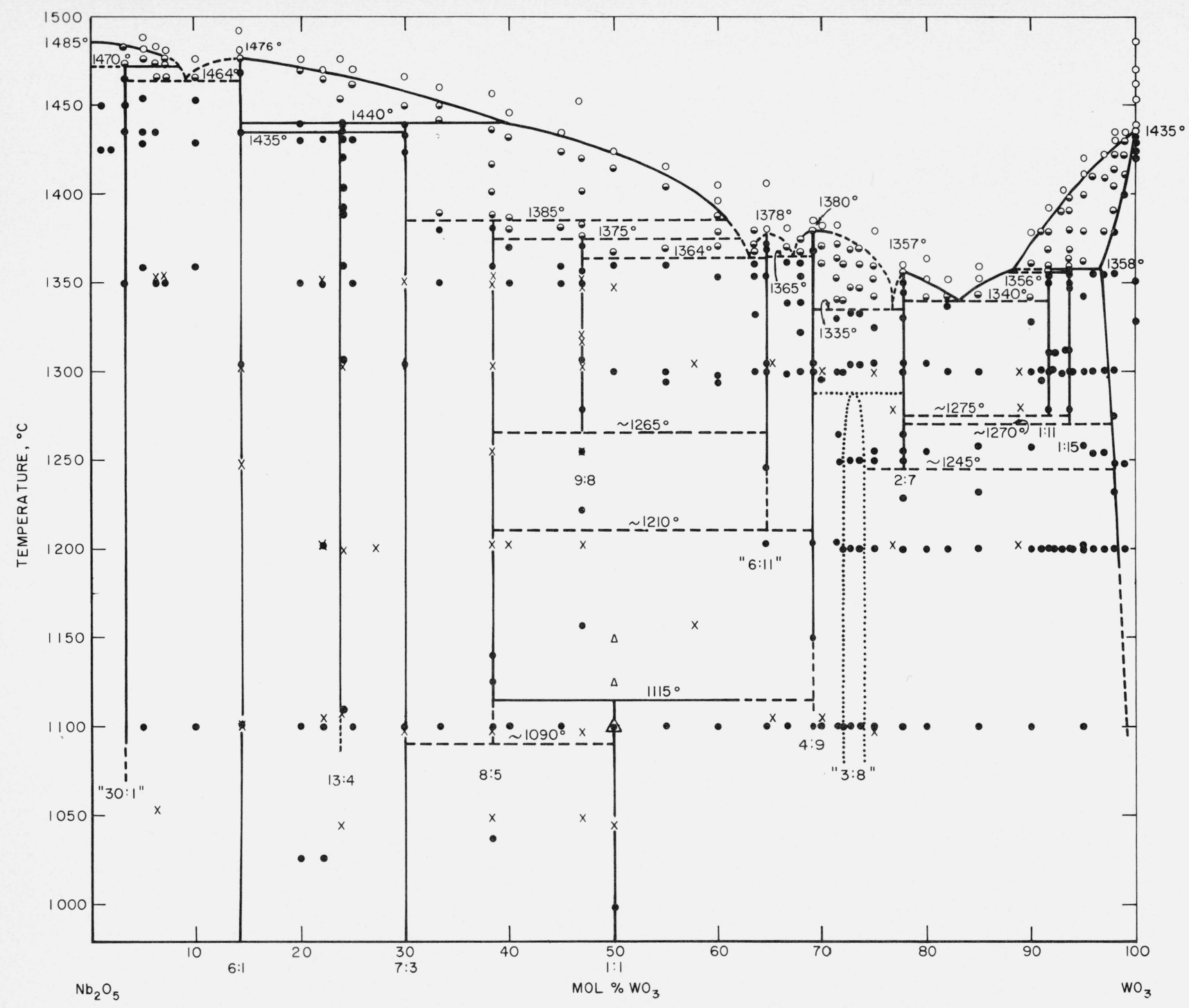

Figure 1. Postulated phase equilibrium diagram of the binary system $\mathrm{Nb}_{2} \mathrm{O}_{5}-\mathrm{WO}_{3}$.

Heavy lines represent well-established portions of the system and dashed lines represent not as well-established portions. Dotted lines represent persistent metastable equilibrium. - not melted.

- partially melted.

- completely melted.

$\times$-experimental data obtained from "less-pure" materials.

$\triangle$ - high-temperature x-ray data.

Tungsten trioxide has been found to accept a maximum of about 3 mole percent $\mathrm{Nb}_{2} \mathrm{O}_{5}$ in solid solution at the solidus temperature of $1358{ }^{\circ} \mathrm{C}$. The amount of solid solution decreases with decreasing temperature to about 2 mole percent at $1230{ }^{\circ} \mathrm{C}$ and less than 1 mole percent at $1100{ }^{\circ} \mathrm{C}$. The melting point of $\mathrm{WO}_{3}$ was found, in the present study, to be $1435{ }^{\circ} \mathrm{C}$. This value was independently confirmed by $\mathrm{E}$. M. Levin [36] and is in disagreement with the previously published value of $1472{ }^{\circ} \mathrm{C}$ (Jaeger and Germs) [37]. In a discussion of the $1472{ }^{\circ} \mathrm{C}$ value for the melting point of $\mathrm{WO}_{3}$, Phillips and Chang [38] concluded that this temperature was probably too low and might actually represent the eutectic between $\mathrm{WO}_{3}$ and $\mathrm{W}_{20} \mathrm{O}_{58}$. However, they did not attempt to experi- mentally determine the melting points of the compounds $\mathrm{WO}_{3}$ and $\mathrm{W}_{20} \mathrm{O}_{58}$ or the solidus temperature between them. The $1435{ }^{\circ} \mathrm{C}$ value for the melting point of $\mathrm{WO}_{3}$ is internally consistent with the experimental phase diagram of both the $\mathrm{Nb}_{2} \mathrm{O}_{5}-\mathrm{WO}_{3}$ and $\mathrm{WO}_{3}-\mathrm{B}_{2} \mathrm{O}_{3}$ systems [36]. This value apparently represents the practical melting point for the experimental conditions utilized: sealed $\mathrm{Pt}$ tube, heated for very short intervals of time $(\leqq 5 \mathrm{~min})$. This method is preferable to that of Jaeger and Germs [37] who utilized an open $\mathrm{Pt}$ crucible and much longer heating time. However, the true melting point of $\mathrm{WO}_{3}$ can probably only be established by utilizing an oxygen pressure furnace and extrapolating to one atmosphere pressure. 
TABLE 19. Experimental data for compositions in the binary system $\mathrm{Nb}_{2} \mathrm{O}_{5}-\mathrm{WO}_{3}$. Part I

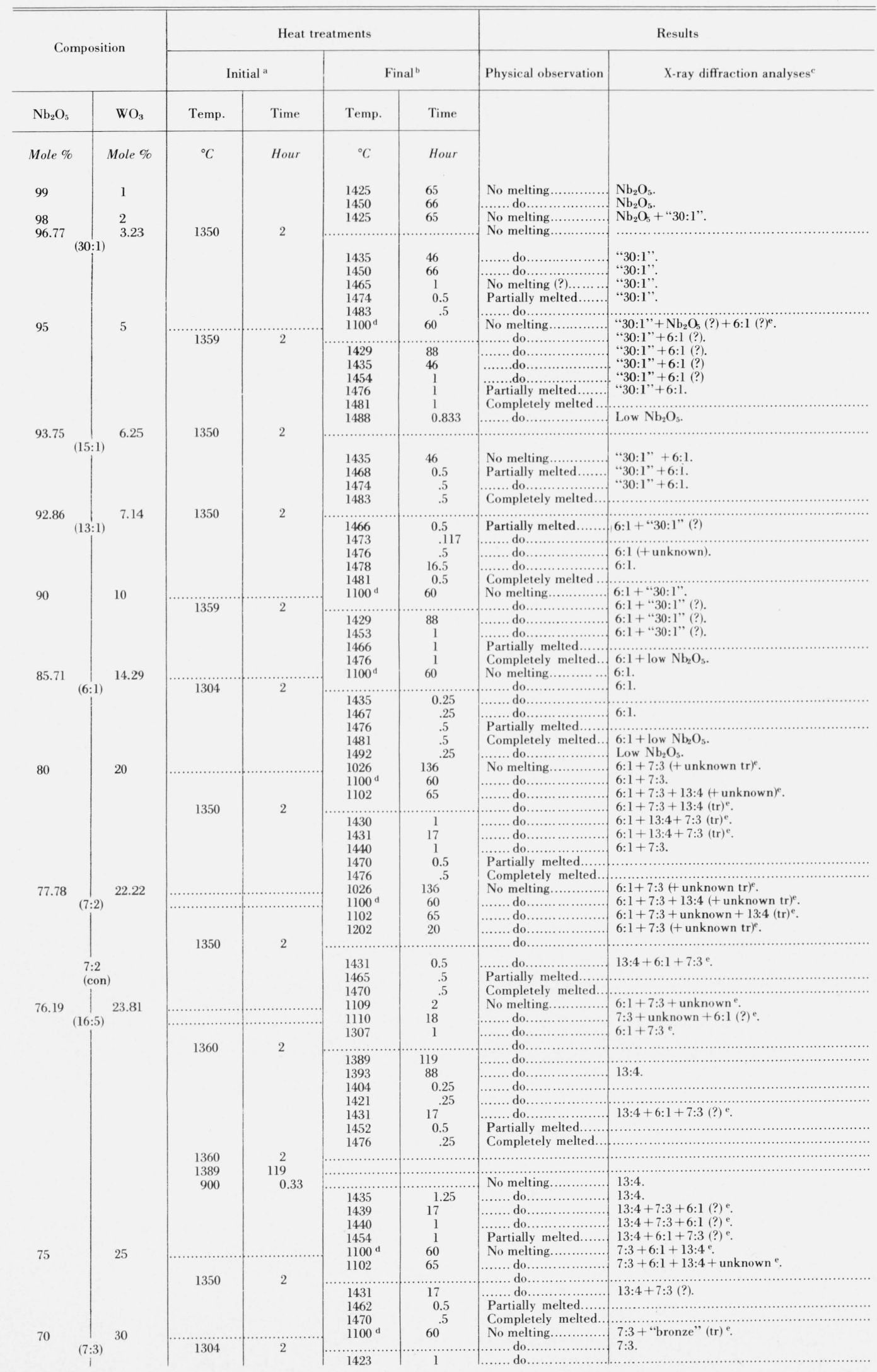


TABLE 19. Experimental data for compositions in the binary system $\mathrm{Nb}_{2} \mathrm{O}_{5}-\mathrm{WO}_{3}$. Part I-Continued.

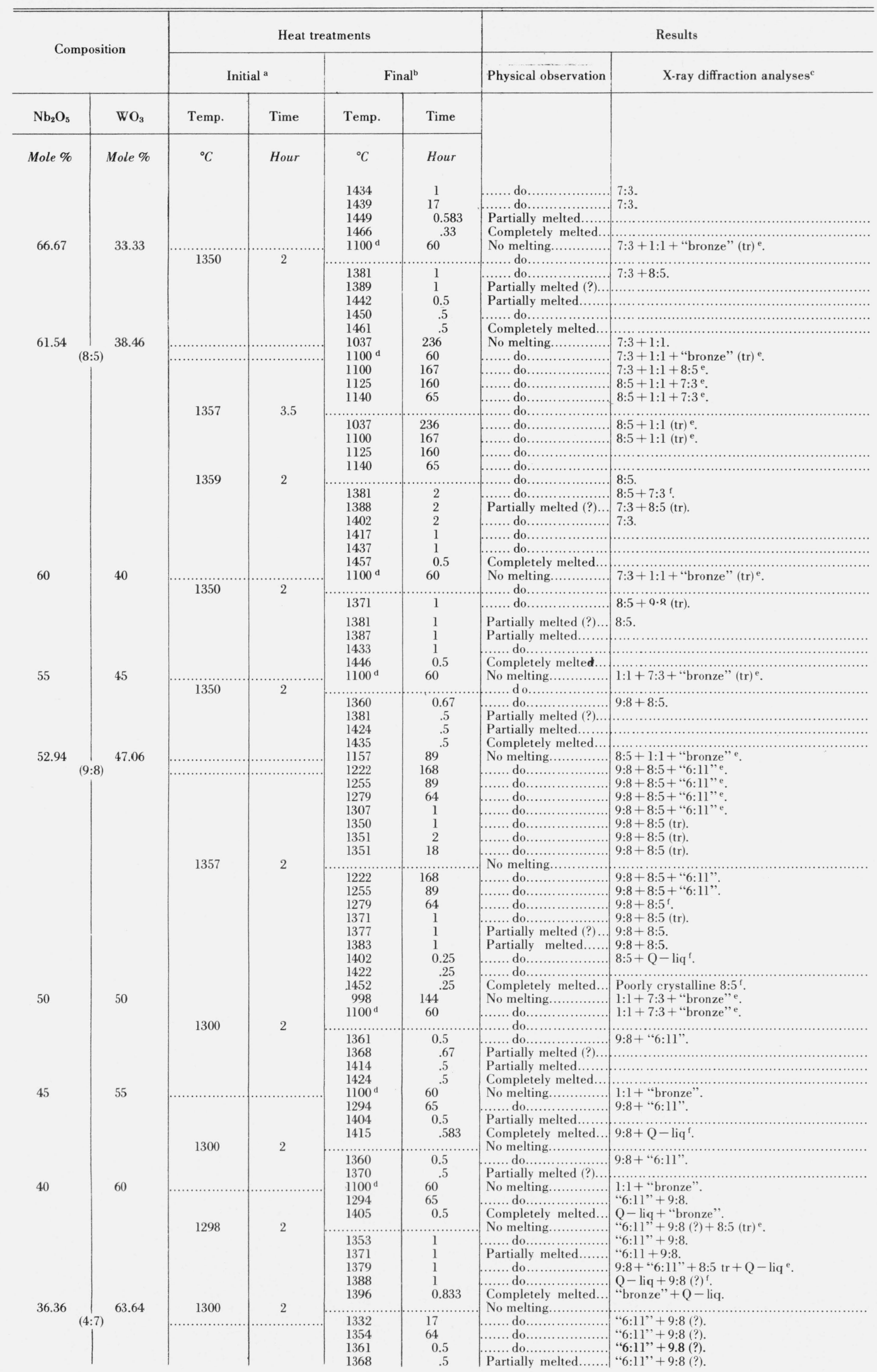


TABLE 19. Experimental data for compositions in the binary system $\mathrm{Nb}_{2} \mathrm{O}_{5}-\mathrm{WO}_{3}$. Part I-Continued.

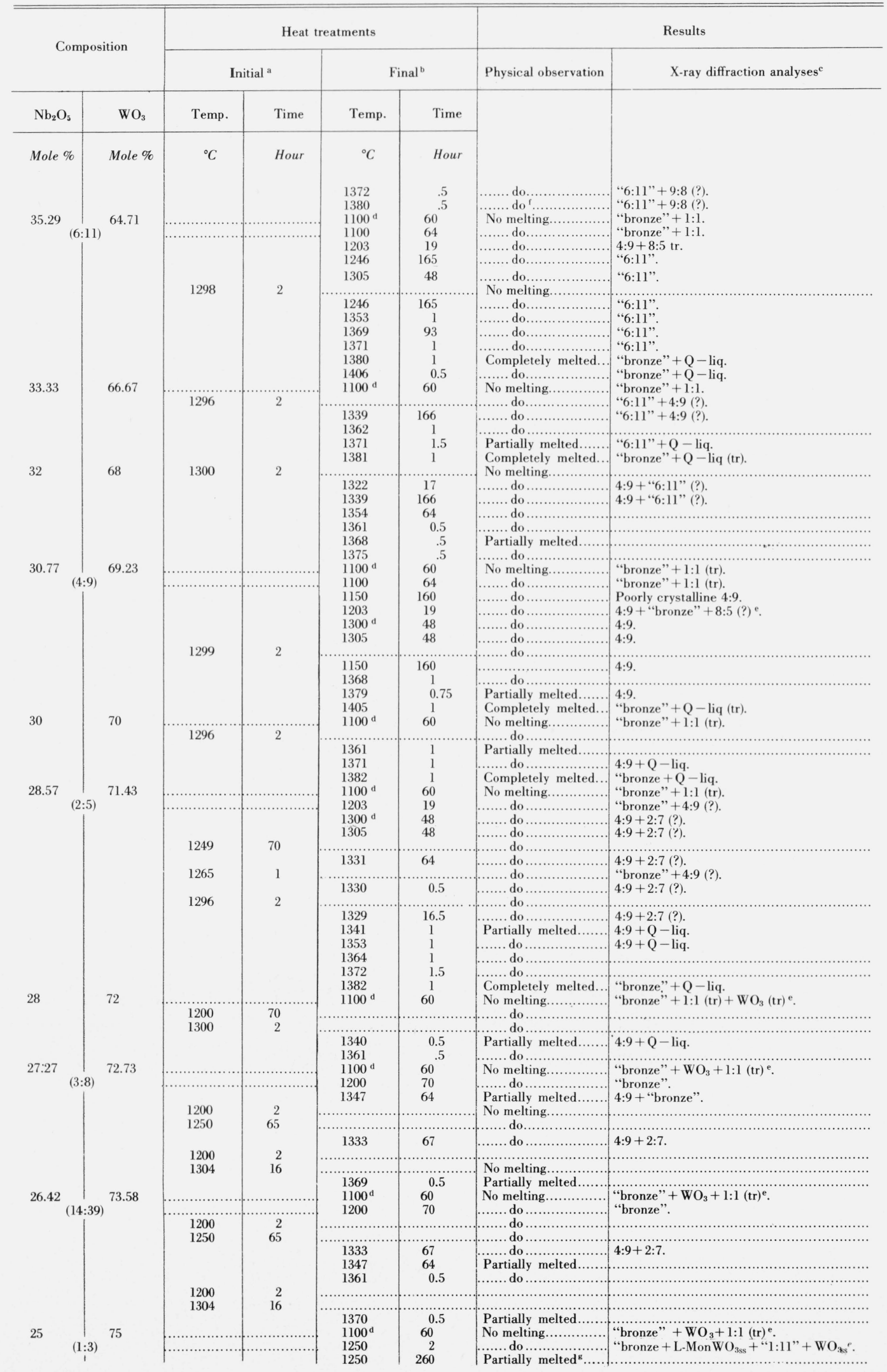


TABLE 19. Experimental data for compositions in the binary system $\mathrm{Nb}_{2} \mathrm{O}_{5}-\mathrm{WO}_{3}$. Part I-Continued.

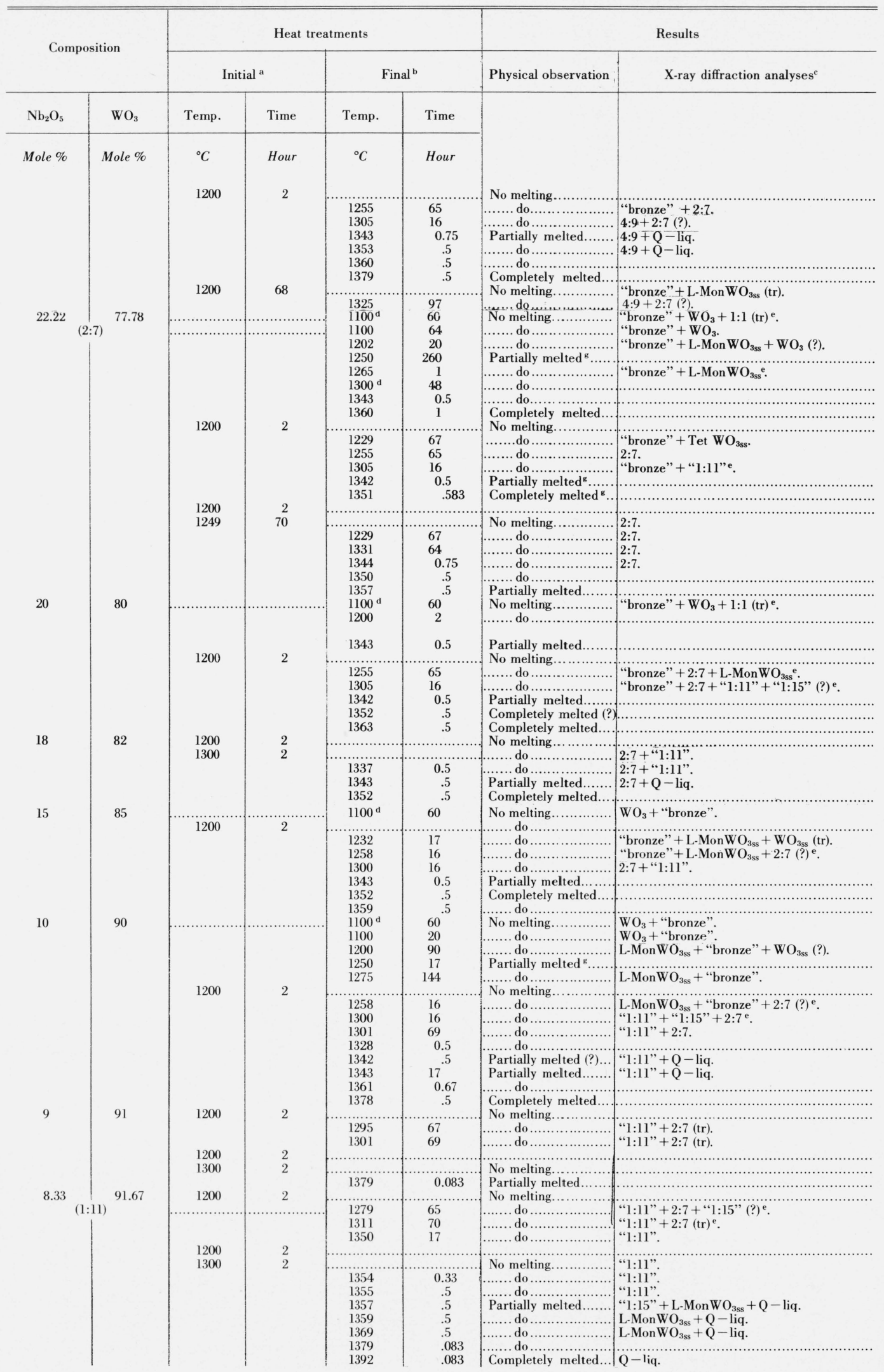


TABLE 19. Experimental data for compositions in the binary system $\mathrm{Nb}_{2} \mathrm{O}_{5}-\mathrm{WO}_{3}$. Part I-Continued.

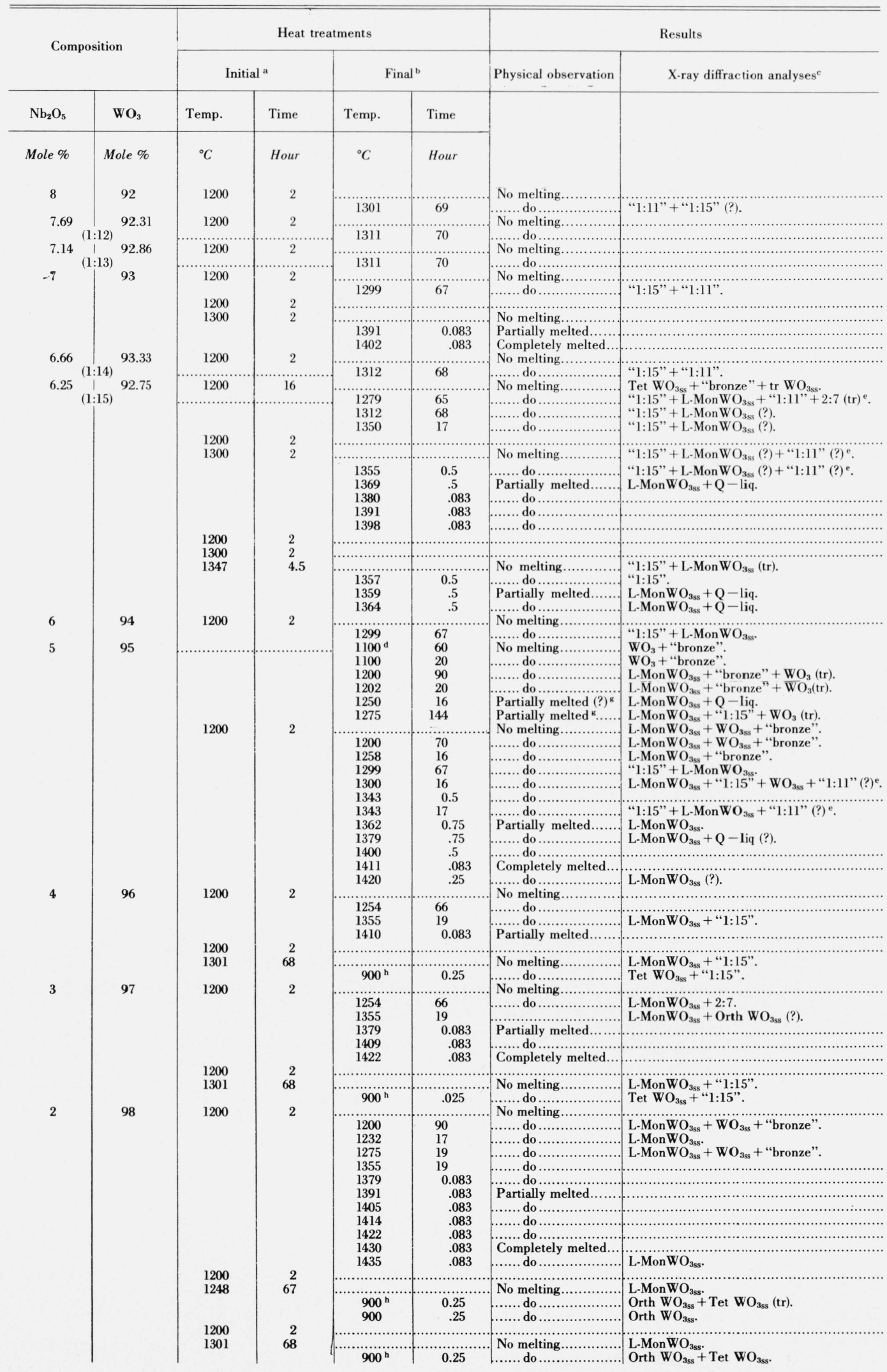


TABLE 19. Experimental data for compositions in the binary system $\mathrm{Nb}_{2} \mathrm{O}_{5}-\mathrm{WO}_{3}$. Part I-Continued.

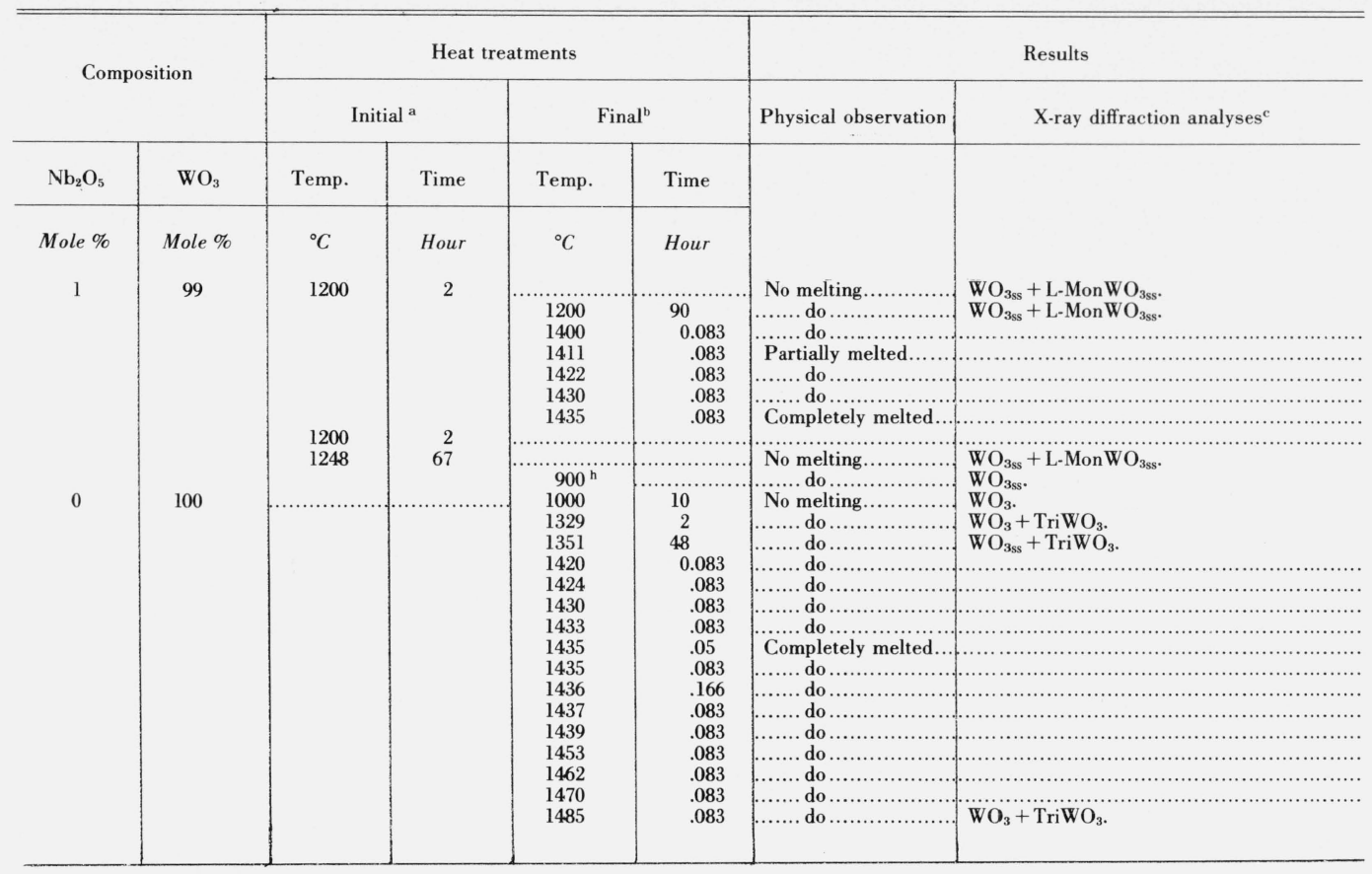

Experimental data for compositions in the binary system $\mathrm{Nb}_{2} \mathrm{O}_{5}-\mathrm{WO}_{3}$. Part II (less pure end members)

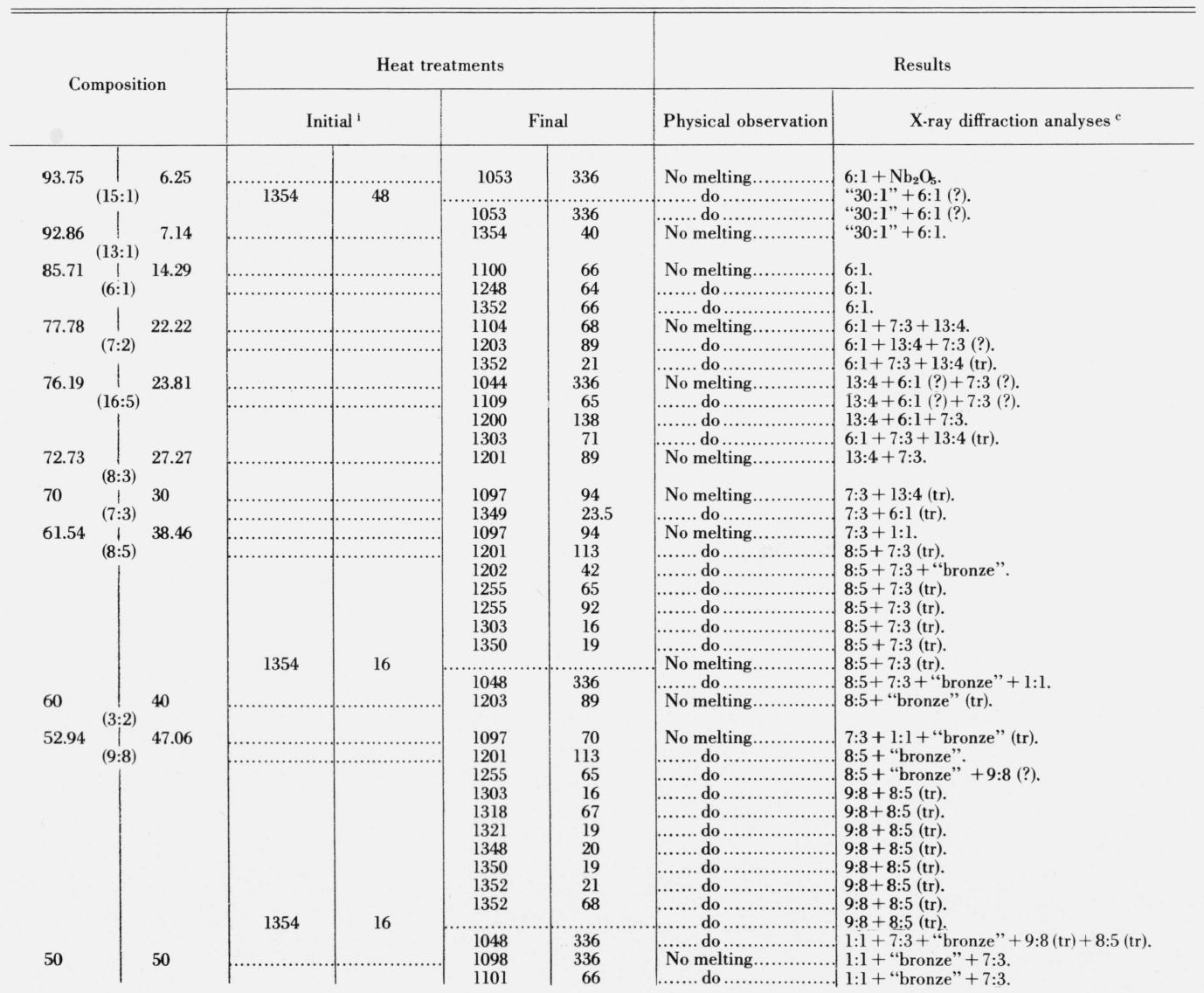


Experimental data for compositions in the binary system $\mathrm{Nb}_{2} \mathrm{O}_{5}-\mathrm{WO}_{3}$. Part II (less pure end members) Continued.

\begin{tabular}{|c|c|c|c|c|c|c|c|}
\hline \multirow{2}{*}{\multicolumn{2}{|c|}{ Composition }} & \multicolumn{4}{|c|}{ Heat treatments } & \multicolumn{2}{|r|}{ Results } \\
\hline & & \multicolumn{2}{|c|}{ Initial $^{i}$} & \multicolumn{2}{|c|}{ Final } & Physical observation & X-ray diffraction analyses ${ }^{c}$ \\
\hline \multirow{3}{*}{\multicolumn{2}{|c|}{$42.31 \underset{(11: 15)}{\mid} 57.69$}} & 1348 & 20 & & & ....... do ................... & \multirow{11}{*}{ 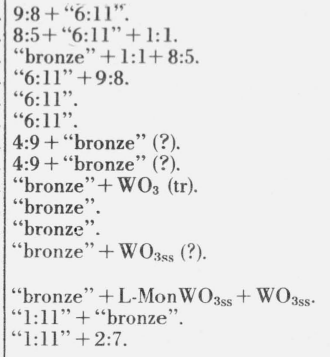 } \\
\hline & & & & $\begin{array}{l}1044 \\
1157\end{array}$ & $\begin{array}{r}350 \\
89\end{array}$ & No melting.............. & \\
\hline & & & & 1305 & 22 & ....... do ...................... & \\
\hline 35.14 & 64.86 & & & 1105 & 67 & No melting.................. & \\
\hline & & & & 1305 & 22 & ....... do ..................... & \\
\hline 30 & 70 & & & $\begin{array}{l}1105 \\
1301\end{array}$ & $\begin{array}{l}67 \\
70\end{array}$ & $\begin{array}{r}\text { No melting } \ldots \ldots \ldots \ldots \ldots \\
\ldots \ldots \ldots \text { do } \ldots \ldots \ldots \ldots \ldots \ldots \ldots \ldots\end{array}$ & \\
\hline & 75 & & & 1097 & 70 & 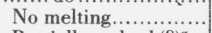 & \\
\hline & & & & $\begin{array}{l}1300 \\
1300\end{array}$ & $\begin{array}{l}44 \\
70\end{array}$ & $\begin{array}{c}\text { Partially melted }(?)^{\mathrm{g}} \ldots \\
\ldots \ldots \ldots \text { do }\end{array}$ & \\
\hline 23.17 & 76.83 & & & 1202 & 24 & No melting............... & \\
\hline & & & & 1279 & 46 & Partially melted $(?)^{\mathrm{g}}$ & \\
\hline 11.11 & 88.89 & & & $\begin{array}{l}1202 \\
1279 \\
1300\end{array}$ & $\begin{array}{l}24 \\
46 \\
44\end{array}$ & $\begin{array}{l}\text { No melting, } \ldots \ldots \ldots \ldots \\
\text { Partially melted }(?)^{g} . \ldots \\
\ldots \ldots \ldots \text { do } \ldots \ldots \ldots \ldots \ldots \ldots \ldots\end{array}$ & \\
\hline
\end{tabular}

a All specimens were initially calcined at $700{ }^{\circ} \mathrm{C}$ for $19 \mathrm{hr}$ with heating and cooling rates of approximately $4{ }^{\circ} \mathrm{C} / \mathrm{min}$. Small portions of this calcine were then reheated for the designated time at one or more designated higher temperatures and generally quenched in sealed $\mathrm{Pt}$ tubes.

${ }^{\mathrm{b}}$ After the initial heat treatment(s) all specimens were reheated at the indicated temperature and quenched in sealed $\mathrm{Pt}$ tubes, unless otherwise specified.

${ }^{c}$ The phases identified are given in the order of amount present at room temperature. The phases are not necessarily those present at the temperature to which the specimen was heated.

$(?)=$ interpretation is not certain

ss = solid solution.

$\begin{aligned} \mathrm{s} & =\text { solid solution. } \\ (\mathrm{tr}) & =\text { trace just barely discernible in } \mathrm{x} \text {-ray pattern }\end{aligned}$

" $30: 1 "$ = a phase of unknown structure occurring at a mole ratio of approximately $30: 1 \mathrm{Nb}_{2} \mathrm{O}_{5}: \mathrm{WO}_{3}$.

(unknown) = extra peaks in the $\mathrm{x}$-ray pattern which cannot be assigned to any recognized phase.

$6: 1=6 \mathrm{Nb}_{2} \mathrm{O}_{5} \cdot \mathrm{WO}_{3}\left(\mathrm{WNb}_{12} \mathrm{O}_{33}\right)$.

low $\mathrm{Nb}_{2} \mathrm{O}_{5}=$ nonequilibrium phase (similar to the low temperature form of $\mathrm{Nb}_{2} \mathrm{O}_{5}$ ), when quenched from above the liquidus and examined at room temperature.

$7: 3=7 \mathrm{Nb}_{2} \mathrm{O}_{5} \cdot 3 \mathrm{WO}_{3}\left(\mathrm{~W}_{3} \mathrm{Nb}_{14} \mathrm{O}_{44}\right)$.

$13: 4=13 \mathrm{Nb}_{2} \mathrm{O}_{5} \cdot 4 \mathrm{WO}_{3}\left(\mathrm{~W}_{4} \mathrm{Nb}_{38} \mathrm{O}_{77}\right)$

"bronze" = a metastable disordered phase having a variable composition and an undistorted tetragonal-tungsten-bronze type x-ray pattern (desig. nated as " $3: 8$ " on the phase diagram).

$8: 5=8 \mathrm{Nb}_{2} \mathrm{O}_{5} \cdot 5 \mathrm{WO}_{3}\left(\mathrm{~W}_{5} \mathrm{Nb}_{16} \mathrm{O}_{55}\right)$

$8: 5=8 \mathrm{Nb}_{2} \mathrm{O}_{5} \cdot 5 \mathrm{WO}_{3}\left(\mathrm{~W}_{5} \mathrm{Nb}_{16} \mathrm{O}_{55}\right)$
$9: 8=9 \mathrm{Nb}_{2} \mathrm{O}_{5} \cdot 8 \mathrm{WO}_{3}\left(\mathrm{~W}_{8} \mathrm{Nb}_{18} \mathrm{O}_{69}\right)$

$9: 8=9 \mathrm{Nb}_{2} \mathrm{O}_{5} \cdot 8 \mathrm{WO}_{3}\left(\mathrm{WN}_{8} \mathrm{Ob}_{18}\right)$
$1: 1=\mathrm{Nb}_{2} \mathrm{O}_{5} \cdot \mathrm{WO}_{3}\left(\mathrm{WNb}_{2} \mathrm{O}_{8}\right)$

$\begin{aligned} 1: 1 & =\mathrm{Nb}_{2} \mathrm{O}_{5} \cdot \mathrm{WO}_{3}\left(\mathrm{WNb}_{2} \mathrm{O}_{8}\right) \text {. } \\ 6: 11 \text { " } & \text { a tungsten bronze type phase of orthorhombic symmetry and with superstructure lines indicative of the unit cell being tripled in the }\end{aligned}$ $a$ and $b$ directions.

$\mathrm{Q}-\mathrm{liq}=$ metastable phase or phases which form only from the quenched liquid and give very poorly defined $\mathrm{x}$-ray powder patterns, having the general appearance of the $\mathrm{ReO}_{3}$ subcell characteristic of most of the compounds in this system.

$4: 9=4 \mathrm{Nb}_{2} \mathrm{O}_{5} \cdot 9 \mathrm{WO}_{3}\left(\mathrm{Nb}_{8} \mathrm{~W}_{9} \mathrm{O}_{47}\right)$

$\mathrm{L}-\mathrm{Mon} \mathrm{WO}_{3 \mathrm{ss}}=$ the monoclinic polymorph of $\mathrm{WO}_{3}$ reported [30] to occur below $-40{ }^{\circ} \mathrm{C}$ "stabilized" by a small amount of solid solution of $\mathrm{Nb}_{2} \mathrm{O}_{5}$ in $\mathrm{WO}_{3}$. $2: 7=2 \mathrm{Nb}_{2} \mathrm{O}_{5} \cdot 7 \mathrm{WO}_{3}\left(\mathrm{~W}_{7} \mathrm{Nb}_{4} \mathrm{O}_{31}\right)$.

"1:11" = Magneli "shear" phase having the approximate composition $1 \mathrm{Nb}_{2} \mathrm{O}_{5}: 11 \mathrm{WO}_{3}$

"1:15" = Magneli "shear" phase having the approximate composition $1 \mathrm{Nb}_{2} \mathrm{O}_{5}: 15 \mathrm{WO}_{3}$.

Tet $\mathrm{WO}_{3 \mathrm{ss}}=$ the high temperature polymorph of $\mathrm{WO}_{3}$ stabilized by solid solution of $\mathrm{Nb}_{2} \mathrm{O}_{5}$ in $\mathrm{WO}_{3}$.

Orth $\mathrm{WO}_{3 \mathrm{ss}}=$ the orthorhombic polymorph of $\mathrm{WO}_{3}$ ordinarily stable between about $320^{\circ} \mathrm{C}$ and $\sim 745{ }^{\circ} \mathrm{C}$, "stabilized" by addition of $\mathrm{Nb}_{2} \mathrm{O}_{5}$ in solid solution.

$\mathrm{Tri} \mathrm{WO}_{3}=$ the polymorph of $\mathrm{WO}_{3}$ which is stable just below room temperature and occurs at room temperature when pure $\mathrm{WO}_{3}$ is ground in a mortar and pestle for a short time.

d Specimen heated and cooled at about $4{ }^{\circ} \mathrm{C} / \mathrm{min}$ rather than quenched.

e Nonequilibrium, probably due to incomplete reaction.

${ }^{\mathrm{f}}$ Nonbinary equilibrium, postulated as being due to reduction.

${ }^{\mathrm{g}}$ Metastable melting.

h $\mathrm{Pt}$ tube not sealed.

i Did not receive preliminary $700^{\circ}$ heat treatment. 


\section{Reduction}

As has been previously mentioned there is some tendency for $\mathrm{WO}_{3}$ to reduce on heating to high temperatures, even in the sealed $\mathrm{Pt}$ tubes. This tendency is greatly enhanced by subjecting the specimens to high temperatures for extended periods of time. The problem of reduction of $\mathrm{WO}_{3}$ is apparently carried into the $\mathrm{Nb}_{2} \mathrm{O}_{5}-\mathrm{WO}_{3}$ system to such an extent that the system may not be completely binary at high temperatures.

The experimental data indicate that the system probably remains essentially binary as long as no melting occurs. However, above the solidus, reduction very likely takes place and the measured liquidus values may not represent binary equilibrium. The melting relations around the ordered bronze-type phases, from about 60 to 80 mole percent $\mathrm{WO}_{3}$, were particularly difficult to obtain experimentally. The three compounds at " $6: 11 ", 4: 9$ and $2: 7$ all appeared to melt congruently with binary solidus relations between them. However, the liquidus values appear to form smooth curves between these congruent melting points without any indication of a change in slope toward a eutectic composition. The phenomena is characteristic of a nonbinary system. For the sake of simplicity, the phase diagram has been drawn to indicate the most likely binary relationships and liquidus curves have been dashed to illustrate the uncertainty in values.

Another indication of reduction occurs in the melting relations of the $\mathrm{B}_{n m+1} \mathrm{O}_{3 n m-(n+m)+4}$ homologous series compounds. The phases $\mathrm{W}_{3} \mathrm{Nb}_{14} \mathrm{O}_{44}, \mathrm{~W}_{5} \mathrm{Nb}_{16} \mathrm{O}_{55}$, and $\mathrm{W}_{8} \mathrm{Nb}_{18} \mathrm{O}_{69}$ were all observed to melt incongruently. Experimentally these compound compositions were observed to begin to form a second phase at about $1440^{\circ}, 1385^{\circ}$, and $1375^{\circ}$, respectively. However, compositions in two-phase regions between these compounds did not begin to form the new phase until considerably higher temperatures, even though the specimen may have shown an appearance of partial melting. This apparent increase in the stability temperature of the phases is dependent on the amount of time the specimen is held at temperature, and binary melting relationships can be approximated by only holding the experimental specimens for relatively short periods of time. It may therefore be concluded that reduction takes place in these specimens, when held above the solidus temperatures. However, it has previously been concluded [1-4] that the crystal structure of these compounds precludes any appreciable nonstoichiometry. Therefore, it appears likely that, as the $\mathbb{W}^{+6}$ ion is reduced, the $\mathrm{Nb} / \mathrm{W}$ ratio of these phases shifts towards higher tungsten content, maintaining an essentially constant cation: anion ratio.

In addition to the relatively large amount of reduction which apparently takes place above melting, there is possibly some tendency towards reduction at lower temperatures. The amount of reduction which can take place experimentally below melting is probably several orders of magnitude less than that which occurs above melting because it seems in no way to affect the equilibrium relationships. The only indication of this minor reduction is in a tendency for discoloration of the specimens. Almost any composition in the system, held for long periods of time in a sealed Pt tube will show a much darker green or blue color than does the same composition either held for shorter periods or not sealed. This darker color can always be lightened by annealing the specimen in an open Pt tube, at about $1000^{\circ} \mathrm{C}$.

It is intriguing to postulate that the $\mathrm{Nb}_{2} \mathrm{O}_{5}-\mathrm{WO}_{3}$ system might more closely approach binary conditions if the experiments were performed under conditions of high oxygen pressure, such as that utilized by Van Hook [39]. Such studies will probably become more common in the near future.

\section{Metastable Phases and Nonequilibrium}

\subsection{Metastable Melting}

It can be seen from table 19, that all compositions containing more than about 72 mole percent $\mathrm{WO}_{3}$ were calcined for about $2 \mathrm{hr}$ at $1200{ }^{\circ} \mathrm{C}$ prior to attempting to obtain equilibrium data. This was because the few preliminary heat treatments, performed at temperatures above $1250{ }^{\circ} \mathrm{C}$, without the $1200{ }^{\circ} \mathrm{C}$ calcine, exhibited some indication of melting, whereas those with a prior $1200{ }^{\circ} \mathrm{C}$ calcine did not show melting until much higher temperatures.

The metastable melting apparently is due to a eutectic type reaction between the metastable bronzetype solid solution (labeled " $3: 8$ " on fig. 1) and pure $\mathrm{WO}_{3}$. It must be emphasized that in order to obtain equilibrium products in the region 72 to 100 mole percent $\mathrm{WO}_{3}$, this preliminary calcine at $1200{ }^{\circ} \mathrm{C}$ is imperative. Without such preliminary heat treatment, neither the 2:7 compound nor the "Magnelishear" structures can be prepared as single phases.

\subsection{Metastable Phases}

The bronze-type solid solution previously mentioned (" $3: 8$ " of fig. 1) has been interpreted as a metastable phase for several reasons. It appears to be responsible for very low temperature metastable melting which takes place between about 72 and 100 mole percent $\mathrm{WO}_{3}$. The x-ray powder pattern of the bronzetype solid solution shows no superstructure and the phase must contain disordered pentagonal bypyramid polyhedra. It must be concluded that the disordered phase is metastable because it dissociates on heating to two ordered phases. All compositions between about the $6: 11$ and 2:7 ratios, when quenched from above the liquidus exhibit the tetragonal bronze-type "disordered" phase with no indication of any superstructure peaks in the x-ray diffraction powder pattern. In addition, many specimens also show a small amount of a quenched liquid phase which exhibits a poorly defined x-ray pattern characteristic of a disordered $\mathrm{ReO}_{3}$ structure, the basic building unit of all the compounds in the system. 
From about 5 mole percent $\mathrm{WO}_{3}$ to at least 15 mole percent $\mathrm{WO}_{3}$ another metastable phase is formed from the quenched liquid. This phase has an x-ray pattern, characteristic of the orthorhombic lowtemperature form of $\mathrm{Nb}_{2} \mathrm{O}_{5}$. The diffraction pattern shows diffuse peaks and little or no superstructure lines. This phase never appears below the melting point and only forms as a single phase if the quenching temperature is considerably above the liquidus. The ability of a specimen to be quenched as a phase with a powder pattern similar to the low-temperature form of $\mathrm{Nb}_{2} \mathrm{O}_{5}$ seems to be dependent on cooling rate and composition. This phenomena has been previously reported for other niobate systems [23, 40].

As shown in table 19, another unknown phase has been found occasionally in nonequilibrium mixtures between about 7 and 25 mole percent $\mathrm{Nb}_{2} \mathrm{O}_{5}$. All efforts to establish an equilibrium region for this phase have proved negative. It is possible that the unknown compound may represent a complex structure involving a mixture of $3 \times 3$ blocks along with the $3 \times 4$ and/or $4 \times 4$ blocks normally found in other compounds occurring in this composition range. Such a structure would contain a considerable number of $\mathrm{Nb}^{+5}$ ions in tetrahedral coordination and would not be expected to form a stable assemblage.

It has been previously mentioned [1] that $\mathrm{WNb}_{12} \mathrm{O}_{33}$ appears to have a small region of homogeneity, in that the 8: 1 composition showed only a single phase. However, no change in lattice parameters could be detected. This apparent region of solid solution is most likely due to the very similar powder patterns of the $6: 1$ compound and the " $30: 1$ " phase. A solid solution structure containing either a considerable number of oxygen vacancies or cation interstitials (in either the tetrahedral or octahedral position) would be expected to cause some measurable change in the lattice parameters. One other possible mechanism which has been suggested for solid solution [41] is again raised by the appearance of the "ordered intergrowth" structure of the $13: 4$ compound, and the suggested possible structure of a $25: 1$ or $20: 1 \mathrm{com}$ pound. It is possible that a "disordered intergrowth" of double $3 \times 4$ blocks might occur in association with the isolated $3 \times 4$ blocks of the $\mathrm{WNb}_{12} \mathrm{O}_{33}$ compound [24]. Such a disordered structure or "compositional stacking fault" would cause only a slight broadening of some of the lines of an x-ray diffraction powder pattern, as would a mixture of a small amount of the ordered second phase. It is problematical as to whether such a picture of solid solution would represent a stable or only a metastable condition. Nevertheless, proof of such a postulated disorder would necessarily depend upon other data, outside the scope of this project. A careful electron diffraction and/or electron microscope study might prove of value in deciphering the nature of any possible disorder of this type.

\section{References}

[1] R. S. Roth and A. D. Wadsley, Acta Cryst. 19, 26 (1965).

[2] R. S. Roth and A. D. Wadsley, Acta Cryst. 19, 32 (1965).

[3] R. S. Roth and A. D. Wadsley, Acta Cryst. 19, 38 (1965).

[4] R. S. Roth and A. D. Wadsley, Acta Cryst. 19, 42 (1965).

[5] H. J. Goldschmidt, Metallurgia 62, 241 (1960).

[6] L. M. Kovba and V. K. Trunov, Dokl. Adad. Nauk. SSR 147, 622 (1962).

[7] L. J. Fiegel, G. P. Mohanty, and §. H. Healy, J. Chem. and Eng. Data 9, 365 (1964).

[8] G. P. Mohanty and L. J. Fiegel, Acta Cryst. 1 7, 454 (1964).

[9] L. M. Kovba, V. K. Trunov, and Y. P. Simanov, Zh. Neorgan. Khim. 9, 1930 (1964).

[10] H. Schäfer and R. Gruehn, private communication to A. D. Wadsley, 4/14/65.

[11] E. J. Felten, private communication to R. S. Roth, 12/2/64.

[12] H. F. Stimson, J. Res. NBS 65A (Phys. and Chem.) No. 3, 139 (1961).

[13] G. Brauer, Z. anorg. u. allgem. Chem. 248, 1 (1941).

[14] F. Holzberg, A. Reisman, M. Berry, and M. Berkenblit, J. Am. Chem. Soc. 79, 2039 (1957).

[15] M. W. Shafer and R. Roy, Z. Krist. 110, 241 (1958).

[16] H. J. Goldschmidt, J. Inst. Metals 87, 235 (1958-59).

[17] A. Reisman and F. Holzberg, J. Am. Chem. Soc. 81, 3182 (1959).

[18] B. M. Gatehouse and A. D. Wadsley, Acta Cryst. 17, 1545 (1964).

[19] R. S. Roth, J. Res. NBS 62, 27 (1959) RP2925.

[20] R. S. Roth and J. L. Waring, J. Res. NBS 66A (Phys. and Chem.) No. 6, 451 (1962).

[21] R. S. Roth, A. D. Wadsley, and S. Andersson, Acta Cryst. 18, 643 (1965).

[22] R. Gruehn and H. Schäfer, Naturwissenschaften 50, 642 (1963).

[23] J. L. Waring and R. S. Roth, J. Res. NBS 69A (Phys, and Chem.) No. 2, 119 (1965).

[24] A. D. Wadsley, S. Andersson, and W. G. Mumme, Acta Cryst. (to be published).

[25] S. Andersson, Acta Chem. Scand. 18, 2339 (1964).

[26] M. Lundberg and S. Andersson, to be published.

[27] A. Sleight and A. Magneli, Acta Chem. Scand. 18, 2007 (1964).

[28] N. Stephensen, private communication.

[29] A. Magneli, Arkiv Kemii 1, 513 (1950).

[30] R. Veda and T. Ichinokawa, Phys. Rev. 82, 563 (1951).

[31] J. Wyart and M. Föex, Compt. Rend. 2459 (1951).

[32] S. Tanisaki, J. Phys. Soc. Japan 15, 566 (1960).

[33] B. T. Mathias and E. A. Wood, Phys. Rev. 84, 1255 (1951).

[34] S. Levine, R. Corwin, and H. L. Blood, Bull. Am. Phys. Soc. Series II, 1, [5], 255 (1956).

[35] P. H. Fang, private communication.

[36] E. M. Levin, J. Am. Ceram. Soc. 48, 491 (1965).

[37] F. M. Jaeger and H. C. Germs, Z. anorg. u. allgem. Chem. 190, 145 (1921).

[38] B. Phillips and L. Chang, Trans. Met. Soc. AIME 230, 1203 (1964).

[39] H. J. Van Hook, Rev. Sci. Instr. 36, 1119 (1965).

[40] E. M. Levin, J. Research NBS 70A (Phys. and Chem.), No. 1, 5 (1966).

[41] A. D. Wadsley, in Non-stoichiometric Compounds, ed. L. Mandelcorn ch. 3, (Academic Press, N.Y., 1964).

(Paper 70A4-404) 